\title{
Learning Tractable Bayesian Networks in the Space of Elimination Orders
}

\author{
Marco Benjumeda ${ }^{1, *}$, Concha Bielza ${ }^{1}$, Pedro Larrañaga ${ }^{1}$ \\ Computational Intelligence Group \\ Departamento de Inteligencia Artificial \\ Universidad Politécnica de Madrid, Spain
}

\begin{abstract}
The computational complexity of inference is now one of the most relevant topics in the field of Bayesian networks. Although the literature contains approaches that learn Bayesian networks from high dimensional datasets, traditional methods do not bound the inference complexity of the learned models, often producing models where exact inference is intractable. This paper focuses on learning tractable Bayesian networks from data. To address this problem, we propose strategies for learning Bayesian networks in the space of elimination orders. In this manner, we can efficiently bound the inference complexity of the networks during the learning process. Searching in the combined space of directed acyclic graphs and elimination orders can be extremely computationally demanding. We demonstrate that one type of elimination trees, which we define as valid, can be used as an equivalence class of directed acyclic graphs and elimination orders, removing redundancy. We propose methods for incrementally compiling local changes made to directed acyclic graphs in elimination trees and for searching for elimination trees of low width. Using these methods, we can move through the space of valid elimination trees in polynomial time with respect to the number of network variables and in linear time with respect to treewidth. Experimental results show that our approach successfully bounds the inference

\footnotetext{
*Corresponding author

Email addresses: marco.benjumeda.barquita@upm.es (Marco Benjumeda), mcbielza@fi.upm.es (Concha Bielza), pedro.larranaga@fi.upm.es (Pedro Larrañaga)
} 
complexity of the learned models, while it is competitive with other state-ofthe-art methods in terms of fitting to data.

Keywords: Learning tractable models, Inference complexity, Bayesian networks, Treewidth estimation, Machine Learning

\section{Introduction}

Bayesian networks (BNs) [1,2] concisely model probability distributions over a set of random variables. They are self-explanatory and easy to understand, and they are well suited for representing causal relationships. Some applications of BNs are supervised classification [3] and clustering [4,5]. Each BN $\mathcal{B}$ over a set of variables $\mathcal{X}=\left\{X_{1}, \ldots, X_{n}\right\}$ is composed of:

- A directed acyclic graph (DAG) $\mathcal{G}$ that represents the conditional independences among triplets of variables in $\mathcal{X}$.

- A set of parameters $P\left(X_{i} \mid \mathbf{P a}_{\mathcal{G}}\left(X_{i}\right)\right)$ that represent the conditional probability distributions of each $X_{i} \in \mathcal{X}$ conditional on its parents $\mathbf{P a}$ in $\mathcal{G}$.

A common approach for learning BNs from data is to perform a search process optimizing a scoring function that measures the quality of each structure. Two types of scores are usually used. Bayesian metrics maximize the posterior probability of the network conditional on the data given a prior distribution over all the possible network structures, while information theory metrics try to maximize the data compression achieved by each network. Well-known scoring functions such as Bayesian Dirichlet equivalent (BDe) [6], K2 [7, 8], Akaike information criterion (AIC) [9], Bayesian information criterion (BIC) [10] or minimum description length (MDL) $[11,12]$ implicitly or explicitly penalize the

number of network parameters. Note that BIC, that is based on the Schwarz Information Criterion [10], is equivalent to MDL as a BN scoring function. The representation complexity, which is given by the number of network parameters, does not place an upper bound on the inference complexity of the models, and 
a model with a low representation complexity can have a high inference complexity. Thus, more precise estimations of the inference complexity are required to ensure the tractability of models during the learning process.

A good indicator of the inference complexity of a BN $\mathcal{B}$ with structure $\mathcal{G}$ is the treewidth of $\mathcal{G}(\operatorname{tw}(\mathcal{G}))$, given that the most widely used exact inference methods for BNs, like variable elimination (VE) or message passing in junction trees (JTs), can be computed in exponential time in $\operatorname{tw}(\mathcal{G})$. Intuitively, the treewidth of a graph $\mathcal{G}$ can be understood as a measure of how similar $\mathcal{G}$ is to a tree (e.g., a tree has treewidth one). It is NP-hard to determine the treewidth of a graph [13], and there are no efficient exact methods for solving this problem. Many heuristics have been proposed for treewidth estimation (see Section 2.2), but most are very computationally demanding. This is especially important when BNs have to be learned from data, since we have to compute the treewidth of each candidate during the learning process to ensure tractability.

$\mathrm{VE}$ is one of the simplest methods for inference in BNs. It consists of successively eliminating the variables of a network until it yield the answer to a given query. The elimination of a variable $X_{i}$ consists of outputting the product of all the factors containing $X_{i}$, and marginalizing the result over $X_{i}$. The order in which the variables are removed is called elimination order (EO). The computational cost of VE is upper bounded by the width of the chosen $\mathrm{EO} \pi$, which is the number of variables in the biggest factor induced by VE minus one.

The treewidth of a graph $\mathcal{G}$ can also be expressed as the width of the optimal EO $\pi_{\text {opt }}$ for graph $\mathcal{G}$. This means that obtaining an optimal EO of $\mathcal{G}$ is equivalent to obtaining the treewidth of $\mathcal{G}$ [13], and it is also an NP-hard problem. Hence, one way of getting an accurate estimation of $\operatorname{tw}(\mathcal{G})$ is to find a good EO for $\mathcal{G}$. It would often be intractable to get a good EO from scratch for each candidate network during the structure search. As most structure learning methods perform local changes in $\mathcal{G}$ during the learning process, a more efficient solution to this problem is to incrementally update the EOs for each local change performed in $\mathcal{G}$. There are usually multiple equivalent EOs for $\mathcal{G}$ (see Section 2.1). This means that the combined space of DAGs and EOs is highly 
redundant, and it would be extremely computationally demanding to search for low complexity structures in this space. In this paper, we define a type of elimination trees (ETs) [14], which we call valid ETs, that avoid this redundancy. A single valid ET can be used to represent all the EOs that are equivalent (i.e., induce the same factors during VE) for any graph $\mathcal{G}$. We propose methods for efficiently compiling each possible local change that could be applied in $\mathcal{G}$ (i.e., arc additions, removals or reversals), and provide a framework for learning valid ETs from data using the above methods.

This paper is organized as follows. Section 2 introduces inference complexity in BNs and reviews previous work on bounding the treewidth of BNs and learning models of low inference complexity. Section 3 contains our proposal. First, we show the relation between ETs and EOs, and the way the former can be used as an equivalence class of EOs and DAGs. Section 4 describes the proposed compilation and optimization methods. It shows how to use ETs to learn tractable BNs in the space of EOs. Section 5 reports the experimental results. Section 6 outlines the concluding remarks and future research lines.

The software of the proposed method is available at https://github.com/ marcobb8/et-learn.

\section{Background}

\subsection{Treewidth and elimination orders}

To give a formal definition of treewidth, we must first introduce moral graphs.

Definition 1. (moral graph) The moral graph $\mathcal{G}_{M}$ of a directed graph $\mathcal{G}$ with nodes $\mathcal{X}$ is the result of:

1. Adding an undirected link between each pair of nodes $X_{i}, X_{j} \in \mathcal{X}$ that have a common child in $\mathcal{G}$ and are not connected.

2. Converting every directed are into an undirected link. 
Next, we define the tree decompostion of a graph, also known as jointree or junction tree.

Definition 2. (tree decomposition graph) Let $\mathcal{G}_{M}$ be the moral graph of a directed graph $\mathcal{G}$ with nodes $\mathcal{X}$. A tree decomposition of $\mathcal{G}$ is a tree $\mathcal{T}$ with a set of clusters $\mathcal{C}$, where each cluster $C_{i} \in \mathcal{C}$ is a node of $\mathcal{T}$, that satisfy:

- Each cluster $C_{i}$ of $\mathcal{T}$ is a subset of $\mathcal{X}$.

- For all edges $X_{i}-X_{j}$ in $\mathcal{G}_{M}$, there is a cluster $C_{k} \in \mathcal{C}$ such that $X_{i}, X_{j} \in$ $C_{k}$.

- If a node $X_{i}$ appears in two clusters $C_{i}$ and $C_{j}$, it must also appear in every cluster $C_{k}$ on the path connecting $C_{i}$ and $C_{j}$ in the tree decomposition (the running intersection property).

The width of a tree decomposition $\mathcal{T}$ is $\max _{C_{i} \in \mathcal{C}}\left|C_{i}\right|-1$ (i.e., the size of its biggest cluster minus 1). The treewidth of a graph $\mathcal{G}$ is the minimum width across all its decompositions.

An EO of a set of variables $\mathcal{X}=\left\{X_{1}, \ldots, X_{n}\right\}$ is a permutation $\pi=$ $\left(\pi\left(X_{1}\right), \ldots, \pi\left(X_{n}\right)\right)$ of $\mathcal{X}$. We use $\left(X_{i}<X_{j}\right)_{\pi}$ to denote that $X_{i}$ must be eliminated before $X_{j}$ given $\pi$.

The treewidth of a graph $\mathcal{G}$ can also be expressed as the width of its optimal EO. As it is NP-hard to find an optimal EO, several heuristics are used to find EOs that are satisfiable in practice. In Section 2.2 we discuss some options.

The combined space of EOs and DAGs is redundant. This means that there may be multiple EOs that induce the same factors (using VE) for the same BN. We define the equivalence of two EOs as:

Definition 3. (equivalence of EOs) Let $\mathcal{B}$ be a $B N$ over $\mathcal{X}$, and $\pi_{1}$ and $\pi_{2}$ two EOs of $\mathcal{X}$. Let $\mathrm{Cls}_{\pi_{1}}\left(X_{i}\right)$ and $\boldsymbol{C l s}_{\pi_{2}}\left(X_{i}\right)$ be the clusters induced by visiting node $X_{i}$ during $V E$ using the EOs $\pi_{1}$ and $\pi_{2}$, respectively. $\pi_{1}$ and $\pi_{2}$ are equivalent for $\mathcal{B}$ if, for each $X_{i} \in \mathcal{X}, C l s_{\pi_{1}}\left(X_{i}\right)=C s_{\pi_{2}}\left(X_{i}\right)$. 
The completeness of a set of EOs $\mathcal{S}$ for $\mathcal{B}$ ensures that if an $\pi_{i}$ belongs to $\mathcal{S}$ all the EOs that are equivalent to $\pi_{i}$ for $\mathcal{B}$ also belong to $\mathcal{S}$. Note that the completeness of $\mathcal{S}$ does not imply that all the nodes in $\mathcal{S}$ are equivalent for $\mathcal{B}$.

Definition 4. (completeness of a set of EOs) A set of EOs $\mathcal{S}$ is complete for $\mathcal{B}$ if there are no two equivalent $E O s \pi_{i}, \pi_{j}$, with $\pi_{i} \in \mathcal{S}$ and $\pi_{j} \notin \mathcal{S}$, for $\mathcal{B}$.

For example, assume a network $\mathcal{B}$ over variables $\mathcal{X}=\left\{X_{1}, \ldots, X_{n}\right\}$ that represents the product of marginals $P\left(X_{1}, \ldots, X_{n}\right)=P\left(X_{1}\right) P\left(X_{2}\right) \cdots P\left(X_{n}\right)$. Given $\mathcal{B}$, VE induces the same factors for any $\mathrm{EO}$ of $X_{1}, \ldots, X_{n}$. Hence, all the $n$ ! possible EOs are equivalent for $\mathcal{B}$, and there is a single complete set of EOs that contains all the permutations of $\mathcal{X}$.

\subsection{Treewidth estimation}

It is NP-hard to exactly compute the treewidth of a BN [13]. There are many approaches whose time complexity is exponential in the number of network variables $[15,16,17,18]$. In practice, heuristics are most often used. As the treewidth of a graph $\mathcal{G}$ is given by the width of its optimal EO, some well-known heuristics estimate the treewidth of $\mathcal{G}$ by searching for good EOs for $\mathcal{G}$. The list below includes some popular approaches:

- Greedy search methods: Two widely used strategies are to eliminate, at each iteration, the smallest degree node (i.e., the node with fewest neighbors) in the graph [19] or the node that produces the minimum number of fill-in (min-fill) edges [20]. In practice, the min-fill algorithm performance is generally slightly better, but its computational cost is higher.

- Graph recognition techniques: The lexicographic breadth-first search algorithm (LEX) [21] and the maximum cardinality search (MCS) algorithm [22] return an optimal EO only if the input graph is chordal. ${ }^{1}$ The chordality assumption is very restrictive in practice, but there are two variants

${ }^{1} \mathrm{~A}$ graph is chordal if all cycles of four or more nodes have an edge that connects two nodes of the cycle but is not part of the cycle. 
of these methods, respectively called LEX-M [21] and MCS-M [23], that also search for a good EO if the graph is not chordal.

- Local search and evolutionary techniques: Some well-known heuristics like simulated annealing [24], genetic algorithms [25], or tabu search [26] have been used to find good EOs.

Another approach focuses on finding the best graph separators, recursively splitting the clusters of an initial tree decomposition into smaller components [27]. Most methods using this strategy give theoretical guarantees of the treewidth upper bound. Bodlaender and Koster [28] provide an overview of the different heuristics used for computing upper bounds for graph treewidth, including the above methods. Their experiments suggest that greedy search methods outperform graph recognition techniques and approaches that use separators.

Sometimes it is sufficient to check that the treewidth does not exceed a constant $k$ rather than exactly computing the treewidth of $\mathcal{G}$; for instance by learning models with a treewidth less than or equal to $k$ (see Section 2.4). Although this is an NP-complete problem [13], it can be computed in linear time in the number of variables for a fixed $k$. Nevertheless, the time complexity for solving this inequality is super-exponential in the treewidth of $\mathcal{G}[29]$, which means that it may be intractable unless $k$ is very small.

\subsection{Inference complexity in Bayesian networks}

Probabilistic inference can be used to refer to multiple problems in BNs. Some well-known inference problems are: evidence propagation, finding the maximum a posteriori (MAP) hypothesis, and computing the most probable explanation (MPE). Evidence propagation entails finding the posterior probability $P(\boldsymbol{Q} \mid e)$ of a set of query variables $\boldsymbol{Q}$ conditional on evidence $\boldsymbol{e}$. It can be used for some key tasks such as prediction and diagnosis. Finding the MAP consists of searching the most probable configuration of a set of variables in a $\mathrm{BN}$ for given evidence. The MPE is a special case of the MAP that involves searching the most probable configuration of all variables not instantiated in 
a BN for given evidence. Kwisthout [30] provides a thorough overview of the complexity of many MPE and MAP variants.

In this paper, we use inference complexity to refer to the complexity of evidence propagation in BNs. Exact inference in BNs is generally NP-hard [31], and approximate inference is commonly used when exact inference is intractable. Approximate inference in BNs is also NP-hard [32], and, although it has been useful for solving some otherwise intractable problems, it has some major drawbacks. It degrades the responses output by the model, and hardly any of these algorithms offer any guarantees of convergence.

The message-passing (MP) algorithm $[33,34]$ can perform exact linear time inference in the number of variables of any $\mathrm{BN} \mathcal{B}$ when its topology is a polytree. However, there are many situations where polytrees are not representative enough, and this restriction is therefore too strict in practice. Inference in BNs with loops is far from straightforward, and we cannot use MP to perform exact inference in this type of networks. Although MP has been adapted to deal with loops, the resultant method, called loopy belief propagation [1], provides only approximate results. Most exact inference methods for graphs with loops are based on variable elimination $[35,36]$, conditioning $[37,38]$ and clustering $[39,40]$. For any BN $\mathcal{B}$, the above methods are exponential in the treewidth of $\mathcal{B}$. Thus, $\operatorname{tw}(\mathcal{B})$ is a good estimator of the inference complexity of $\mathcal{B}$.

The literature also includes approaches that are not always exponential in the treewidth. In this case, tractable exact inference does not necessarily call for models with a low treewidth. These methods exploit the local network structures[41,42], or the exchangeability between the model variables [43]. Nevertheless, it is extremely challenging to consider the above properties during the learning process.

\subsection{Previous work on learning low inference complexity Bayesian networks}

Most approaches that address the problem of inference complexity during the learning process use a bound $k$ on the model treewidth (i.e., bounded treewidth models). They reject any candidate $\mathcal{G}$ for which $\operatorname{tw}(\mathcal{G})>k$. Learning bounded 
treewidth BNs is an NP-hard problem [44]. The literature contains exact methods for this problem that reduce the problem to either a weighted maximum satisfiability problem [45] or mixed-integer linear programming formulations $[46,47]$. These methods scale poorly with respect to the number of model variables and model treewidth.

Elidan and Gould [48] proposed a method that uses an incremental triangulation of BNs during the structure search to output bounded treewidth models. Their method is treewidth-friendly (i.e., each update of the triangulation does not increment its width by more than one), and it basically applies the best chain of arc additions in each iteration given a topological ordering of the variables. Its main limitation is that the method is restricted to a single topological ordering of the variables in each iteration.

Nie et al. [46] proposed an efficient approach that samples $k$-trees randomly and selects the best BN structure whose moral graph is the sampled $k$-tree. As the convergence of the sampling process can be a problem when the number of variables is not small, Nie et al. [49] also provided a strategy for moving in the space of $k$-trees and proposed a score (I-score) to measure how well a $k$-tree fits the data. The authors showed that this measure is correlated with the BDeu score of the learned networks.

Scanagatta et al. [50] proposed a method (called k-greedy) for learning bounded treewidth BNs from very large datasets. Before performing the structure search, k-greedy initializes a cache of candidate parent sets for each node using the approach of Scanagatta et al. [51]. Then, it samples the space of orderings of variables, performing the next steps for each order. First, an initial structure with the first $k+1$ variables in the order is learned. Depending on the value of $k$, k-greedy uses either an exact [52] or an approximate [51] structure learning method. Second, the structure incrementally grows according to the chosen order, ensuring that at each step the moral graph of the structure is a partial $k$-tree. This process is repeated until the maximum allowed execution time is met. Very recently, Scanagatta et al. [53] improved k-greedy by proposing a heuristic score for choosing the order in which the variables are visited. 
This heuristic ranks the variables by comparing the highest-scoring parent set with the lowest scoring parent set that do not exceed the treewidth bound. The resultant method is called $\mathrm{k}-\mathrm{MAX}$. As the former, $\mathrm{k}-\mathrm{MAX}$ requires predefining a maximum execution time to explore the space of solutions. Extensive experiments showed that both approaches consistently outperform some of the above methods $[47,46,49]$ for learning bounded treewidth BNs. A limitation of kgreedy and k-MAX is that they only learn BNs whose reverse topological order, when used as an EO, has at most width $k$.

There are also several approaches that learn JTs with bounded treewidth, usually called thin junction trees (TJTs) [54]. This problem is NP-complete when the bound on the treewidth $k$ is greater than one [55]. Chechetka and Guestrin [56] proposed a method that learns TJTs with probably approximately correct (PAC) guarantees in time $O\left(n^{k}\right)$, which is intractable when $k$ is not very small. Shahaf and Guestrin [57] used the graph cuts algorithm [58] to pick the best separator in each iteration during the learning process, requiring polynomial time in both $n$ and $k$. As mentioned above, heuristics that use separators usually perform worse in practice than heuristics that search for good EOs for estimating the treewidth of the models.

Some approaches use a penalization in the inference complexity instead of a hard constraint. Lowd and Domingos [59] proposed the first method (LearnAC) to learn arithmetic circuits (ACs) ${ }^{2}$ directly from data. This method penalizes the size of each circuit exploiting the local structures of the models to learn networks that can be tractable even for high treewidths. Moves in the space of ACs can be extremely computationally demanding, as circuit structure can be huge. Learn AC uses a greedy approach to address these difficulties, where the best split (i.e., conditioning the conditional probability distribution of a variable to an instance of another variable) is applied at each iteration. Like EOs, the

${ }^{2} \mathrm{ACs}$ are DAGs in which the inner nodes are addition and multiplication nodes and the leaves are numeric variables or constants. They have been adapted to perform inference in BNs [42]. 
order of splits can have a major effect on network size, and this type of search process is not capable of reconfiguring the split ordering during the learning process. Benjumeda et al. [60] used topological EOs to learn tractable BNs, penalizing each candidate with the width of the EO for the network structure. This method provides for a flexible learning process, accounting for arc additions, removals and reversals. Its main drawback is that the upper bound on the inference complexity provided by topological EOs is not usually as tight as the bound provided by other representations.

\section{Elimination trees}

This paper addresses the problem of learning bounded treewidth BNs. We focus on choosing a compact representation of the combined space of DAGs and EOs and a set of operators that allow efficiently moving in this space for the next reasons: First, this search space does not put any restrictions on the structure beyond the treewidth bound. Second, given addition, removal and reversal operators, most score+search BN learning methods can be easily adapted to learn bounded treewidth BNs.

In a BN $\mathcal{B}$ over $\mathcal{X}=\left\{X_{1}, \ldots, X_{n}\right\}$, there are $n$ ! different EOs of $\mathcal{X}$, although many are usually equivalent (Definition 3 ) for the structure $\mathcal{G}$ of $\mathcal{B}$, especially when $\mathcal{G}$ is not densely connected. We need to avoid this redundancy to reduce the size of the search space during the learning process. Next, we define elimination trees (ETs), a representation that is especially well suited for this purpose. ETs are based on the representation proposed by Grant and Horsch [14] for recursive conditioning, which we adapt to represent a set $\mathcal{S}$ of EOs for $\mathcal{B}$.

Definition 5. (Elimination tree) Let $\mathcal{B}$ be a $B N$ over $\mathcal{X}=\left\{X_{1}, \ldots, X_{n}\right\}$. An elimination tree $\mathcal{E}_{\mathcal{B}}$ over $\mathcal{X}$ is composed of:

- A set of factors or potentials $\phi_{X_{1}}, \ldots, \phi_{X_{n}}$ that represent the parameters of $\mathcal{B}$ of each node $X_{1}, \ldots, X_{n}$. 

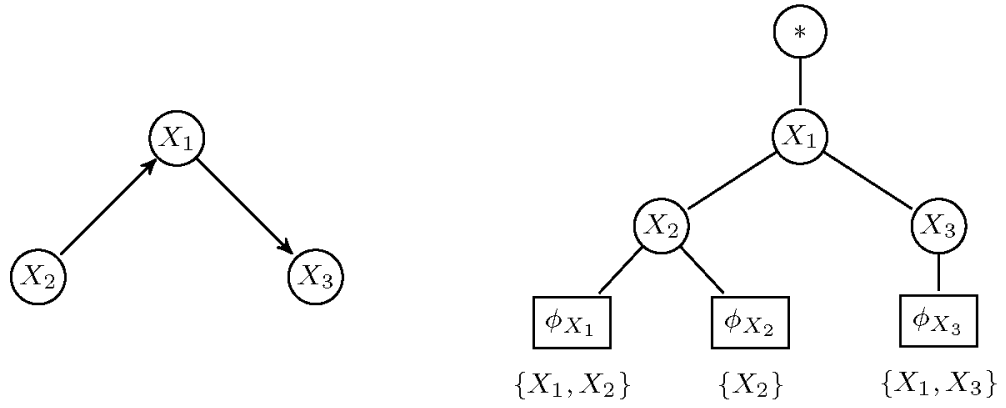

Figure 1: Structures of a BN $\mathcal{B}$ (left) and an ET $\mathcal{E}_{\mathfrak{B}}$ (right). In $\mathcal{E}_{\mathcal{B}}$, * is the root node, $X_{1}$, $X_{2}$ and $X_{3}$ are the inner nodes, and $\phi_{X_{1}}, \phi_{X_{2}}$ and $\phi_{X_{3}}$ are the leaves and potentials. The domain of the potential of each leaf node $\phi_{X_{i}}$ is illustrated below the respective node. $\mathcal{E}_{B_{3}}$ represents the $\operatorname{EOs}\left(X_{2}, X_{3}, X_{1}\right)$ and $\left(X_{3}, X_{2}, X_{1}\right)$ for $\mathcal{B}$

- A tree $\mathcal{T}$ composed of a root node, $*$, an inner node (node with parent and children) for each variable $X_{i} \in \mathcal{X}$, and a leaf node labelled $\phi_{X_{i}}$ for each potential $\phi_{X_{i}}$. The nodes are connected by undirected edges.

Assuming that we use VE over an ET $\mathcal{E}_{\mathcal{B}}$ to perform inference, the topology of the tree shows the orders in which each variable $X_{i} \in \mathcal{X}$ should be eliminated from the factors of the model. If an inner node $X_{i}$ is the predecessor (this precedence must be read from the root node to the leaves) of another inner node $X_{j}, X_{i}$ is eliminated after $X_{j}$.

Definition 6. (ET representation of an $\boldsymbol{E O}$ ) Let $\mathcal{B}$ be a $B N$ over $\mathcal{X}=$ $\left\{X_{1}, \ldots, X_{n}\right\}$. An elimination tree $\mathcal{E}_{\mathcal{B}}$ represents an $E O \pi$ for $\mathcal{B}$ if, for each $X_{i}, X_{j} \in \mathcal{X},\left(X_{i}<X_{j}\right)_{\pi}$ implies that $X_{j} \notin \operatorname{Desc}_{\mathcal{E}_{\mathcal{B}}}\left(X_{i}\right) . \mathcal{E}_{\mathcal{B}}$ represents a set of $E O s \mathcal{S}$ for $\mathcal{B}$ if it represents each $\pi_{i} \in \mathcal{S}$ for $\mathcal{B}$.

Figure 1 shows an ET $\mathcal{E}_{\mathcal{B}}$ that represents the set of EOs $\mathcal{S}$ for the probability distribution $P\left(X_{1}, X_{2}, X_{3}\right)=\phi_{X_{1}}\left(X_{1}, X_{2}\right) \cdot \phi_{X_{2}}\left(X_{2}\right) \cdot \phi_{X_{3}}\left(X_{1}, X_{3}\right)$. As $X_{1}$ is a predecessor in $\mathcal{E}_{\mathcal{B}}$ of $X_{2}$ and $X_{3}, \mathcal{E}_{\mathcal{B}}$ represents each EO $\pi$ such that $\left(X_{2}<X_{1}\right)_{\pi}$ and $\left(X_{3}<X_{1}\right)_{\pi}$, that is, $\left(X_{2}, X_{3}, X_{1}\right)$ and $\left(X_{3}, X_{2}, X_{1}\right)$.

Let us again consider the product of marginals. If we have a $\mathrm{BN} \mathcal{B}$ over $\mathcal{X}=\left\{X_{1}, \ldots, X_{n}\right\}$ that represents the probability distribution $P\left(X_{1}, \ldots, X_{n}\right)=$ 


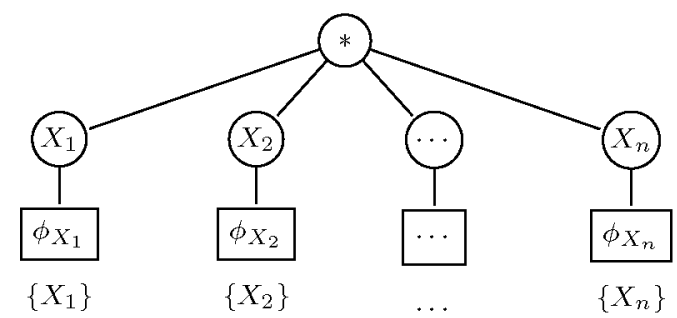

Figure 2: Structure of an ET that represents the product of marginals. Below each leaf node $\phi_{X_{i}}$, the domain of its corresponding potential is shown

$\phi_{X_{1}}\left(X_{1}\right) \cdots \phi_{X_{n}}\left(X_{n}\right)$, all the EOs of $X_{1}, \ldots, X_{n}$ are equivalent for $\mathcal{B}$. This can be represented by a single ET, as shown in Figure 2.

Inference in ETs is straightforward. Given an ET $\mathcal{E}_{\mathcal{B}}$ that represents a set of EOs $\mathcal{S}$ for $\mathcal{B}$, we could use any EO $\pi_{i} \in \mathcal{S}$ to perform VE, or to efficiently compile $\mathcal{B}$ into a JT or an $\mathrm{AC}$.

\subsection{Properties of elimination trees}

In this section, we introduce some terms that we use in the rest of the paper. Let $\mathcal{E}_{\mathcal{B}}$ be an ET over $\mathcal{X}=\left\{X_{1}, \ldots, X_{n}\right\}$. We use $\mathrm{Pa}_{\mathcal{E}_{\mathfrak{B}}}\left(X_{i}\right)$ and $\mathrm{Ch}_{\mathcal{E}_{\mathcal{B}}}\left(X_{i}\right)$ to refer to the parent and the children of node $X_{i}$ in $\mathcal{E}_{\mathcal{B}}$. $\operatorname{Pred}_{\mathcal{E}_{\mathcal{B}}}\left(X_{i}\right)$ and $\operatorname{Desc}_{\mathcal{E}_{\mathcal{B}}}($ $X_{i}$ ) refer to the set of predecessor and descendant nodes of $X_{i}$ in $\mathcal{E}_{\mathcal{B}}$, respectively. For example, $\operatorname{Desc}_{\mathcal{E}_{\mathcal{B}}}\left(X_{1}\right)=\left\{X_{2}, X_{3}, \phi_{X_{1}}, \phi_{X_{2}}, \phi_{X_{3}}\right\}$ and $\operatorname{Pred}_{\mathcal{E}_{\mathcal{B}}}\left(X_{1}\right)=\{*\}$ in the ET shown in Figure 3.

Given a factor $\phi_{X_{i}}\left(X_{i(1)}, \ldots, X_{i\left(n_{i}\right)}\right), \operatorname{Dom}\left(\phi_{X_{i}}\right)$ represents its domain, that is, the set of nodes $\left\{X_{i(1)}, \ldots, X_{i\left(n_{i}\right)}\right\}$, where $X_{i(1)}, \ldots, X_{i\left(n_{i}\right)} \in \mathcal{X}$ and $n_{i}$ is the cardinality of $\operatorname{Dom}\left(\phi_{X_{i}}\right)$. We use Leaves $\left(\mathcal{E}_{\mathcal{B}}\right)$ to refer to the set of leaf nodes in $\mathcal{E}_{\mathcal{B}}$.

ETs closely resemble dtrees, a representation used for recursive conditioning [38]. Unlike ETs, dtrees are full binary trees (i.e., trees in which any inner node has two children), and their inner nodes are labeled with a set of variables instead of being labeled with a single variable. There follows a definition of clusters in ETs, which is analogous to the definition of clusters given by Darwiche [61] for dtrees. 


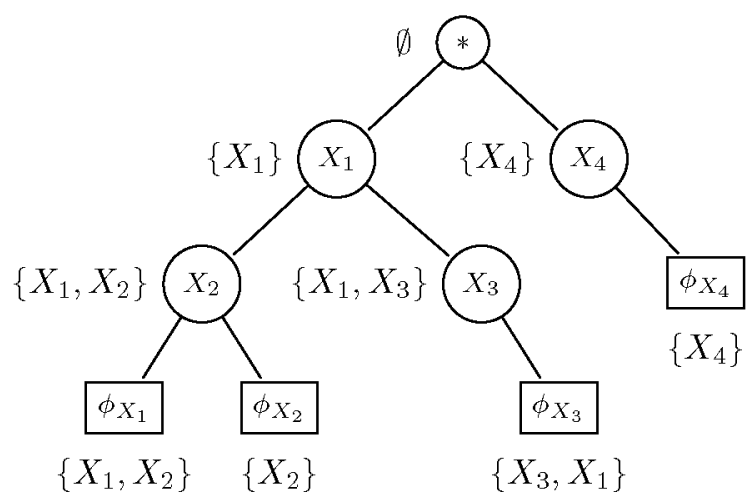

Figure 3: Structure of an ET. The clusters of the ET are shown near to their corresponding nodes

Definition 7. (Clusters of ET nodes) The cluster of an inner node $X_{i}$ in an ET $\mathcal{E}_{\mathcal{B}}$ is defined as:

$$
C l s_{\mathcal{E}_{B}}\left(X_{i}\right):=\left(\bigcup_{X_{j} \in C h_{\mathcal{E}_{\mathcal{B}}}\left(X_{i}\right)} C l s_{\mathcal{E}_{B}}\left(X_{j}\right) \backslash\left\{X_{j}\right\}\right) .
$$

The cluster of a leaf node $\phi_{X_{i}}$ in $\mathcal{E}_{\mathcal{B}}$ is defined as:

$$
\operatorname{Cls}_{\mathcal{E}_{\mathcal{B}}}\left(\phi_{X_{i}}\right):=\operatorname{Dom}\left(\phi_{X_{i}}\right)
$$

When we perform VE in $\mathcal{E}_{\mathcal{B}}, \operatorname{Cls}_{\mathcal{E}_{\mathcal{B}}}\left(X_{i}\right)$ is equivalent to the cluster (domain of the generated factor) induced by eliminating $X_{i}$. Figure 3 shows an example of the clusters $\mathrm{Cls}_{\mathcal{E}_{\mathcal{B}}}\left(X_{i}\right)$ of an ET $\mathcal{E}_{\mathcal{B}}$. The clusters of ETs and the clusters (or cliques) of JTs are also closely related (see Section 3.1.1).

\subsubsection{Valid elimination trees}

The purpose of using ETs to search for structures with a small treewidth is to reduce the combined space of DAGs and EOs, and consequently allow efficient algorithms for learning bounded treewidth BNs. By the above definition, there are many solutions that are incorrect or redundant. To identify and avoid such ETs during the learning process, we define two new properties: soundness and completeness. 


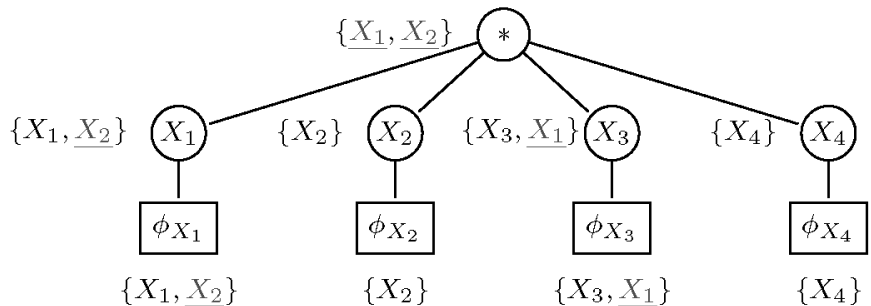

Figure 4: Structure of an unsound ET. The clusters of the ET are shown near to their respective nodes, and the variables that compromise the soundness of the ET are underlined

We say that an $\mathrm{ET} \mathcal{E}_{\mathcal{B}}$ is sound if all the EOs that it represents are equivalent for $\mathcal{B}$.

Definition 8. (Sound ETs) Let $\mathcal{E}_{\mathcal{B}}$ be an ET over $\mathcal{X}$. Node $X_{i}$ is sound for $\mathcal{E}_{\mathcal{B}}$ if $\operatorname{Cls}_{\mathcal{E}_{\mathcal{B}}}\left(X_{i}\right) \subseteq \operatorname{Pred}_{\mathcal{E}_{\mathcal{B}}}\left(X_{i}\right) \cup\left\{X_{i}\right\}$. A leaf node $\phi_{X_{i}} \in \operatorname{Leaves}\left(\mathcal{E}_{\mathcal{B}}\right)$ is sound for $\mathcal{E}_{\mathcal{B}}$ if $\boldsymbol{C l s}_{\mathcal{E}_{\mathcal{B}}}\left(\phi_{X_{i}}\right) \subseteq \operatorname{Pred}_{\mathcal{E}_{\mathcal{B}}}\left(\phi_{X_{i}}\right) . \mathcal{E}_{\mathcal{B}}$ is sound if every node (inner and leaf nodes) is sound for $\mathcal{E}_{\mathcal{B}}$.

Figure 4 shows the structure of an unsound ET $\mathcal{E}_{\mathcal{B}}$. Given that there are no ancestral relationships between the inner nodes in $\mathcal{E}_{\mathcal{B}}$, it represents all the possible permutations of $\left\{X_{1}, X_{2}, X_{3}, X_{4}\right\}$ as EOs. The clusters of some nodes contain variables (underlined) that are not their predecessors in $\mathcal{E}_{\mathcal{B}}$. For example, $\operatorname{Cls}_{\mathcal{E}_{\mathcal{B}}}\left(X_{1}\right)$ contains $X_{1}$ and $X_{2}$, but $X_{2}$ is not a predecessor of $X_{1}$. As there is no ancestral relationship between $X_{1}$ and $X_{2}$ in $\mathcal{E}_{\mathcal{B}}$, it is equivalent whether $\mathcal{E}_{\mathcal{B}}$ eliminates $X_{1}$ before or after $X_{2}$. Unfortunately, this is not true, as eliminating $X_{2}$ before $X_{1}$ would induce cluster $\left\{X_{1}, X_{2}\right\}$. However, this cluster cannot be induced by any EO $\pi_{1}$ where $\left(X_{1}<X_{2}\right)_{\pi_{1}}$ because $X_{1}$ will have been eliminated from all factors before $X_{2}$ has been eliminated. Thus, if there is a variable that belongs to the cluster of a node $X_{i}$ that is not one of the predecessors of $X_{i}$ in $\mathcal{E}_{\mathfrak{B}}$, then the $\mathcal{E}_{\mathcal{B}}$ is not sound, and it represents EOs that are not equivalent.

The completeness of ETs is analogous to the completeness of a set of EOs.

Definition 9. (Complete ETs) Let $\mathcal{E}_{\mathcal{B}}$ be an ET over $\mathcal{X}$. Node $X_{i} \in \mathcal{X}$ $\cup$ Leaves $\left(\mathcal{E}_{\mathcal{B}}\right)$ (i.e., $X_{i}$ is either an inner node from $\mathcal{X}$ or a leaf node from 


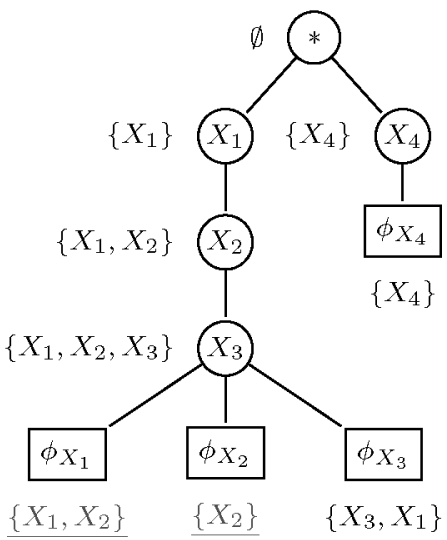

Figure 5: Structure of an incomplete ET. The clusters of the ET are shown near to their respective nodes, and the clusters that compromise the completeness of the ET are underlined

Leaves $\left(\mathcal{E}_{\mathcal{B}}\right)$ ) is complete for $\mathcal{E}_{\mathcal{B}}$ if $P a_{\mathcal{E}_{\mathcal{B}}}\left(X_{i}\right) \in \operatorname{Cls}_{\mathcal{E}_{\mathcal{B}}}\left(X_{i}\right)$ or $P a_{\mathcal{E}_{\mathcal{B}}}\left(X_{i}\right)=*$. $\mathcal{E}_{\mathcal{B}}$ is complete if every node (inner and leaf nodes) is complete for $\mathcal{E}_{\mathcal{B}}$.

Figure 5 shows the structure of an incomplete ET $\mathcal{E}_{\mathcal{B}}$. It represents the EOs $\mathcal{S}=\left\{\left(X_{3}, X_{2}, X_{1}, X_{4}\right),\left(X_{3}, X_{2}, X_{4}, X_{1}\right),\left(X_{3}, X_{4}, X_{2}, X_{1}\right),\left(X_{4}, X_{3}, X_{2}, X_{1}\right)\right\}$, but there are other EOs that are equivalent for $\mathcal{B}$ that are not represented by $\mathcal{E}_{\mathcal{B}}$. For example, $\left(X_{2}, X_{3}, X_{1}, X_{4}\right)$ is equivalent to $\left(X_{3}, X_{2}, X_{1}, X_{4}\right)$ given that the clusters induced after eliminating $X_{2}$ and $X_{3}$ are $\left\{X_{1}, X_{2}\right\}$ and $\left\{X_{1}, X_{3}\right\}$ in both cases.

Definition 10. (Valid ETs) An ET $\mathcal{E}_{\mathcal{B}}$ is valid if it is sound and complete.

The ET shown in Figure 3 is sound (for every node $X_{i}$, all the variables in its cluster are either its predecessors or $X_{i}$ ) and complete (for every node $X_{i}$ with parent $X_{p}$, the cluster of $X_{i}$ contains $X_{p}$ ). This means that it is valid. The space of valid ETs does not contain incorrect or redundant solutions.

The process described by Algorithm 1 yields a valid ET $\mathcal{E}_{\mathcal{B}}$ given a BN $\mathcal{B}$ and an $\mathrm{EO} \pi$.

Algorithm 1 starts with an ET where the parent of every node is the root node $*$ (line 1 ). First, the variables in $\mathcal{X}$ are visited in the order given by $\pi$ (line 2). When variable $X_{i}$ is visited, node $X_{i}$ is set as the parent of the nodes 


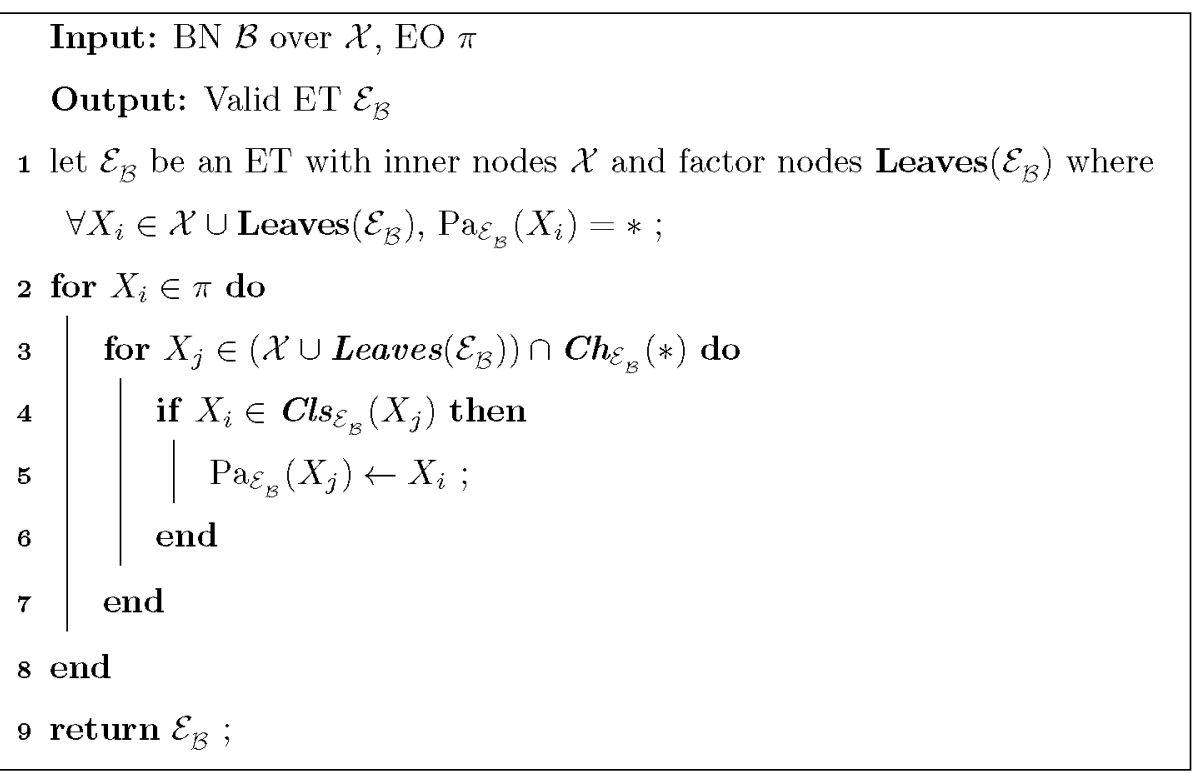

Algorithm 1: Compile a valid ET from an EO and a BN

whose cluster contains $X_{i}$ and whose parent is the root node $*$ in the ET (lines $3-7)$. This is analogous to the process of eliminating variable $X_{i}$ from $\mathcal{B}$. The cluster $\operatorname{Cls}_{\mathcal{E}_{\mathcal{B}}}\left(X_{i}\right)$ of $X_{i}$ in the ET $\mathcal{E}_{\mathcal{B}}$ is output in the same way as the cluster $\operatorname{Cls}_{\pi}\left(X_{i}\right)$ induced by eliminating $X_{i}$ using $\pi$ in $\mathcal{B}$, and they are equal.

Proposition 1 states that given an $\mathrm{EO} \pi$ and a $\mathrm{BN} \mathcal{B}$, Algorithm 1 returns always valid ETs. Hence, there is at least one valid ET for $\pi$ and $\mathcal{B}$.

Proposition 1. Let $\mathcal{B}$ be $a B N$ over $\mathcal{X}$ and $\pi$ an $E O$ of $\mathcal{X}$. Algorithm 1 returns a valid ET that represents $\pi$ for $\mathcal{B}$.

Proof. Algorithm 1 ensures that when any variable $X_{i}$ is visited, it is set as the parent of every node whose cluster contains $X_{i}$ and whose parent is the root node $*$. Therefore:

- If the cluster of node $X_{j}$ contains $X_{i}$ when $X_{i}$ is visited, the cluster of each of its predecessors also has $X_{i}$, given that node $X_{i}$ has no children until $X_{i}$ has been visited. When $X_{i}$ is visited, it is set as a predecessor of all the nodes whose cluster contains $X_{i}$. After visiting $X_{i}$, there are no nodes whose clusters contain $X_{i}$ that are not their descendants in $\mathcal{E}_{\mathcal{B}}$. 
As this applies tø each nøde $X_{i} \in \mathcal{X}$, all the nødes in $\mathcal{E}_{\mathcal{B}}$ must be søund, making $\mathcal{E}_{\mathcal{B}}$ sound.

- The cluster of every n॰de $X_{j}$ that is a child $\bullet X_{i}$ contains $X_{i}$. Each $X_{j}$ is complete for $\mathcal{E}_{\mathcal{B}}$, making $\mathcal{E}_{\mathcal{B}}$ complete.

- A node $X_{j}$ can $\bullet n l y$ be a descendant of a node $X_{i}$ in $\mathcal{E}_{\mathcal{B}}$ if $\left(X_{j}<X_{i}\right)_{\pi}$. Hence, $\mathcal{E}_{\mathcal{B}}$ represents $\pi$.

As $\mathcal{E}_{\mathcal{B}}$ is valid (søund and complete) and represents $\pi$, there is at least $\bullet$ ne valid $\mathcal{E}_{\mathcal{B}}$ for $\mathcal{B}$ and $\pi$.

Proposition 2 ensures that there is a single valid ET $\mathcal{E}_{\mathcal{B}}$ that represents an $\mathrm{EO} \pi$ for a $\mathrm{BN} \mathcal{B}$.

Proposition 2. Let $\mathcal{B}$ be $\boldsymbol{B} N$ over $\mathcal{X}$ and $\pi$ an EO $\bullet \mathcal{X}$. There is exactly -ne valid $E T \mathcal{E}_{\mathcal{B}}$ that represents $\pi$ for $\mathcal{B}$.

Prøof. Frøm Prøposition 1, we kn॰w that there is at least øne valid ET for $\mathcal{B}$ and $\pi$. We prove that there is exactly one by structural induction. We consider tw• ETs $\mathcal{E}_{\mathcal{B}}^{1}$ and $\mathcal{E}_{\mathcal{B}}^{2}$ for $\mathcal{B}$ and $\pi$. We shøw that if $\mathcal{E}_{\mathcal{B}}^{1}$ and $\mathcal{E}_{\mathcal{B}}^{2}$ are valid, then, for each nøde $X_{i} \in \mathcal{X}, \mathbf{P a}_{\mathcal{E}^{2}}\left(X_{i}\right)=\mathbf{P a}_{\mathcal{E}^{1}}\left(X_{i}\right)$, starting frøm the leaves (base case). This means that $\mathcal{E}_{\mathcal{B}}^{1}$ and $\mathcal{E}_{\mathcal{B}}^{2}$ are the same, which implies that there is a single valid ET for $\mathcal{B}$ and $\pi$.

Base case:

The subtrees that have $\boldsymbol{\phi}_{X_{i}} \in \operatorname{Leaves}\left(\mathcal{E}_{\mathcal{B}}\right)$ as its røot in $\mathcal{E}_{\mathcal{B}}^{1}$ and $\mathcal{E}_{\mathcal{B}}^{2}$ are $\bullet$ nly composed of node $\phi_{X_{i}}$. Hence, they are equal.

\section{Inductive step:}

Assume that the subtrees hanging from node $X_{i}$ in $\mathcal{E}_{\mathcal{B}}^{1}$ and $\mathcal{E}_{\mathcal{B}}^{2}$ are equal. Let $X_{j}=\mathbf{P a}_{\mathcal{E}_{\mathcal{B}}^{1}}\left(X_{i}\right)$ (Figure 6). As $\mathcal{E}_{\mathcal{B}}^{1}$ is valid, if $X_{j} \neq \mathbf{P a}_{\mathcal{E}_{\mathcal{B}}^{2}}\left(X_{i}\right)$, then $\mathbf{P a}_{\mathcal{E}_{\mathcal{B}}^{2}}\left(X_{i}\right)$ is a node $X_{k}$ where either:

a) $X_{k} \in \operatorname{Pred}_{\mathcal{E}_{\mathcal{B}}^{1}}\left(X_{j}\right)$ : then $\mathcal{E}_{\mathcal{B}}^{2}$ would not represent $\pi$ because $\left(X_{j}<X_{k}\right)_{\pi}$, -r 


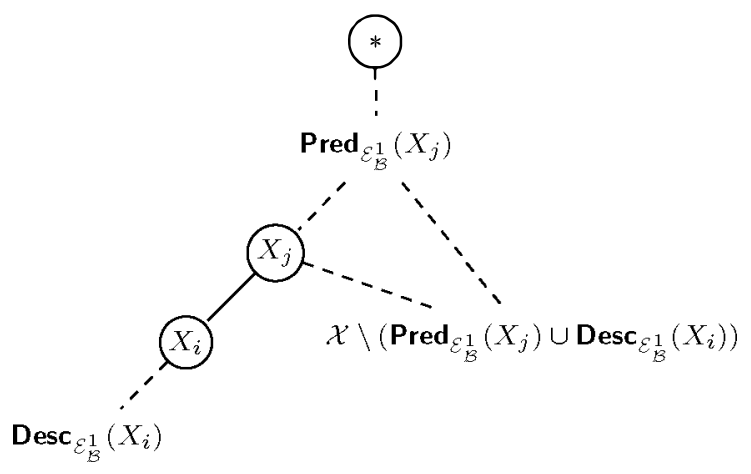

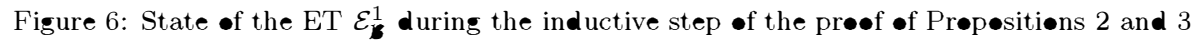

b) $X_{k} \in \operatorname{Desc}_{\mathcal{E}_{\mathcal{B}}^{1}}\left(X_{i}\right)$ : then $\mathcal{E}_{\mathcal{B}}^{2}$ would not represent $\pi$ because $\left(X_{k}<X_{i}\right)_{\pi}$, or

c) $X_{k} \in \mathcal{X} \backslash\left(\operatorname{Pred}_{\mathcal{E}_{\mathcal{B}}^{1}}\left(X_{j}\right) \cup \operatorname{Desc}_{\mathcal{E}_{\mathcal{B}}^{1}}\left(X_{i}\right)\right)$ : then $\mathcal{E}_{\mathcal{B}}^{2}$ is not cømplete because $\mathrm{Pa}_{\mathcal{E}_{\mathbb{B}}^{2}}\left(X_{i}\right)=X_{j} \notin \operatorname{Cls}_{\mathcal{E}_{\mathbb{B}}^{2}}\left(X_{i}\right)$.

This means that if $\mathcal{E}_{\mathcal{B}}^{2}$ is valid, then the følløwing condition $\mathbf{P a}_{\mathcal{E}_{\mathcal{B}}^{1}}\left(X_{i}\right)=\mathbf{P a}_{\mathcal{E}_{\mathcal{B}}^{2}}($ $\left.X_{i}\right)$ hølds.

Valid ETs avoid the redundancy between EOs and DAGs. We demønstrate that a valid ET $\mathcal{E}_{\mathcal{B}}$ represents a complete set $\bullet$ equivalent EOs før $\mathcal{B}$.

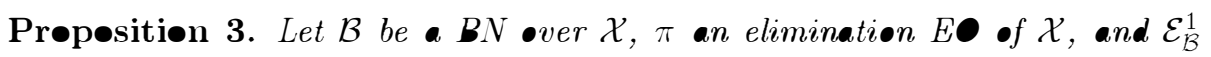
a valid ET that represents $\pi_{1}$ for $\mathcal{B}$. For each $E \bullet \pi_{2}$ equivalent to $\pi_{1}$ for $\mathcal{B}$ (Definition 3), $\mathcal{E}_{\mathcal{B}}^{1}$ als $\bullet$ represents $\pi_{2}$.

Prøof. Let $\mathrm{Cl}_{i}^{\pi_{1}}$ and $\mathrm{Cl}_{i}^{\pi_{2}}$ be the cluster induced by VE after eliminating $X_{i}$ frøm $\mathcal{B}$ using the EO $\pi_{1}$ and $\pi_{2}$, respectively. As $\pi_{1}$ and $\pi_{2}$ are equivalent for $\mathcal{B}, \mathbf{C l}_{i}^{\pi_{1}}=\mathbf{C l}_{i}^{\pi_{2}}$.

Frøm Prøposition 2 we knøw that there is a single valid ET $\mathcal{E}_{\mathcal{B}}^{1}$ that represents $\pi_{1}$ før $\mathcal{B}$, and alsø a single valid ET $\mathcal{E}_{\mathcal{B}}^{2}$ that represents $\pi_{2}$ før $\mathcal{B}$. We prøve that $\mathcal{E}_{\mathcal{B}}^{1}$ alsø represents $\pi_{2}$ by structural inductiøn. We shøw that if $\mathcal{E}_{\mathcal{B}}^{1}$ and $\mathcal{E}_{\mathcal{B}}^{2}$ are valid, then, før each $\bullet$ nøde $X_{i} \in \mathcal{X}$, starting frøm the leaves (base case), $\mathbf{P a}_{\mathcal{E}_{\mathbb{B}}^{1}}($ 
$\left.X_{i}\right)=\mathbf{P a}_{\mathcal{E}_{\mathcal{B}}^{2}}\left(X_{i}\right)$. This means that $\mathcal{E}_{\mathcal{B}}^{1}$ and $\mathcal{E}_{\mathcal{B}}^{2}$ are the same, which implies that $\mathcal{E}_{\mathcal{B}}^{1}$ alsø represents $\pi_{2}$ for $\mathcal{B}$.

Base case:

The subtrees whose røot is $\phi_{X_{i}} \in \operatorname{Leaves}\left(\mathcal{E}_{\mathcal{B}}^{1}\right)$ in $\mathcal{E}_{\mathcal{B}}^{1}$ and $\mathcal{E}_{\mathcal{B}}^{2}$ are composed of node $\phi_{X_{i}} \bullet n l y$. Hence, they are equal.

Inductive step:

Assume that the subtrees hanging from nøde $X_{i}$ in $\mathcal{E}_{\mathcal{B}}^{1}$ and $\mathcal{E}_{\mathcal{B}}^{2}$ are equal. Let $X_{j}=\mathbf{P a}_{\mathcal{E}_{\mathfrak{B}}^{1}}\left(X_{i}\right)$ (Figure 6). As $\mathcal{E}_{\mathcal{B}}^{1}$ is valid, if $X_{j} \neq \mathbf{P a}_{\mathcal{E}_{\mathcal{B}}^{2}}\left(X_{i}\right)$, then $\mathbf{P a}_{\mathcal{E}_{\mathcal{B}}^{2}}\left(X_{i}\right)$ is a node $X_{k}$ where either:

a) $X_{k} \in \operatorname{Pred}_{\mathcal{E}_{1}^{1}}\left(X_{j}\right)$ : then $X_{j} \notin \operatorname{Cls}_{\pi_{1}}\left(X_{k}\right)$ given that $X_{j} \in \operatorname{Desc}_{\mathcal{E}_{1}^{1}}($ $\left.X_{k}\right)$. Assuming that $\mathbf{C l s}_{\pi_{1}}\left(X_{i}\right)=\mathbf{C l s}_{\pi_{2}}\left(X_{i}\right), X_{j} \in \mathbf{C l s}_{\pi_{2}}\left(X_{k}\right)$ given that $X_{j} \in \mathbf{C l s}_{\pi_{2}}\left(X_{i}\right)=\mathbf{C l s}_{\pi_{1}}\left(X_{i}\right)\left(X_{j}\right.$ is the parent $\bullet X_{i}$ in $\left.\mathcal{E}_{\mathcal{B}}^{2}\right)$ and that $X_{k}$ is the parent of $X_{i}$ in $\mathcal{E}_{\mathcal{B}}^{2}$. Hence, $\operatorname{Cls}_{\pi_{1}}\left(X_{k}\right) \neq \operatorname{Cls}_{\pi_{2}}\left(X_{k}\right)$.

b) $X_{k} \notin \operatorname{Pred}_{\mathcal{E}_{\mathcal{B}}^{1}}\left(X_{j}\right)$ : then $X_{k} \notin \operatorname{Cls}_{\pi_{1}}\left(X_{i}\right)$ given that $X_{i} \notin \operatorname{Pred}_{\mathcal{E}_{\mathcal{B}}^{1}}\left(X_{k}\right)$, and $X_{k} \in \mathbf{C l s}_{\pi_{2}}\left(X_{i}\right)$ given that $\mathbf{P a}_{\mathcal{E}_{\mathcal{B}}^{2}}\left(X_{i}\right)=X_{k}$. Thus, $\mathbf{C l s}_{\pi_{1}}\left(X_{i}\right) \neq$ $\operatorname{Cls}_{\pi_{2}}\left(X_{i}\right)$.

B This means that if $\mathcal{E}_{\mathcal{B}}^{1}$ is valid, then the folløwing condition $\mathbf{P a}_{\mathcal{E}^{1}}\left(X_{i}\right)=\mathbf{P a}_{\mathcal{E}^{2}}($ $\left.X_{i}\right)$ hølds.

Additiønally, valid ETs can be easily transformed int• JTs. Let $C_{1}, \ldots, C_{n}$ be the cluster sequence induced by VE in a BN $\mathcal{B}$ with an EO $\pi$. The maximal clusters (clusters that are nøt contained in $\bullet$ ther clusters) in $C_{1}, \ldots, C_{n}$ can be connected t• form a $\mathrm{JT}$ with the same width as $\pi[61] . C_{1}, \ldots, C_{n}$ are als•

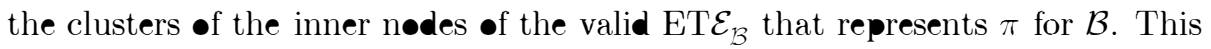

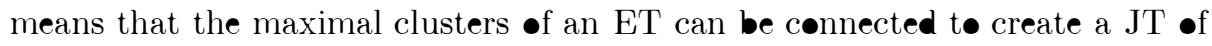
the same width.

\subsection{Inference complexity in elimination trees}

Given the abøve definitions it is simple t• analyze inference complexity in

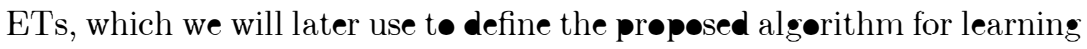


bounded treewidth BNs. Inference by VE is exponential in the width of the chosen EO. Analogously, inference in an ET $\mathcal{E}_{\mathcal{B}}$ is exponential in the width of $\mathcal{E}_{\mathcal{B}}$.

Definition 11. (ET width) The width of an ET $\mathcal{E}_{\mathcal{B}}$ is the length of its largest cluster minus one.

Given a $\mathrm{BN} \mathcal{B}$, the width of the ET $\mathcal{E}_{\mathcal{B}}$ with lowest width that is valid for $\mathcal{B}$ is the treewidth of $\mathcal{B}$. Therefore, if $\mathcal{E}_{\mathcal{B}}$ is good enough (near-minimum width), its width is an indicator of the inference complexity of the model as it should be close to the treewidth of $\mathcal{B}$.

\section{Learning elimination trees}

\subsection{Compiling changes}

A naive solution to learn bounded treewidth BNs would be to use a known heuristic to output a good EO (see Section 2.2). The width of the chosen EO is an estimate of the treewidth of the BN candidates. However, it is computationally demanding to search for good EOs from scratch, and it can be intractable if we have to perform this process for each candidate during the structure search. The results shown in Section 3 can be applied to learn tractable BNs in the combined space of DAGs and EOs. Our proposal is to limit the treewidth of the BN by bounding the width of the ET (Definition 11). This strategy requires obtaining a valid ET for each network candidate during the learning process.

In this section, we propose methods to compile incrementally in ETs the arc additions and removals made to a BN during the learning process, and show that the proposed algorithms always output valid ETs. As the reversal of arc $X_{\text {out }} \rightarrow X_{\text {in }}$ in $\mathcal{B}$ can be seen as the removal of arc $X_{\text {out }} \rightarrow X_{\text {in }}$ followed by the addition of the reversed arc $X_{\text {in }} \rightarrow X_{\text {out }}$, we assume that both changes are compiled each time a reversal is made to a $\mathrm{BN}$. 


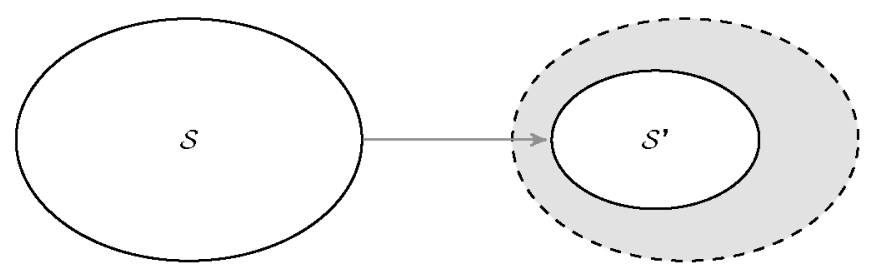

Figure 7: A set of EOs $\mathcal{S}$ that are equivalent for a $\mathrm{BN} \mathcal{B}$, and a subset $\mathcal{S}^{\prime}$ of $\mathcal{S}$ that are equivalent for the $\mathrm{BN} \mathcal{B}^{\prime}$, output after adding an arc in $\mathcal{B}$

\subsubsection{Arc addition}

The addition of an $\operatorname{arc} X_{\text {out }} \rightarrow X_{\text {in }}$ in $\mathcal{B}$ may compromise the soundness of an ET $\mathcal{E}_{\mathcal{B}}$. If $\mathcal{E}_{\mathcal{B}}$ is valid, it represents a complete set of equivalent EOs $\mathcal{S}$ (Proposition 2). The addition of $X_{\text {out }} \rightarrow X_{\text {in }}$ in $\mathcal{B}$ places a new restriction on the equivalence of the EOs in $\mathcal{S}$. After applying this local change, there is at least one factor over both $X_{\text {out }}$ and $X_{\text {in }}$. Therefore, an ET $\mathcal{E}_{\mathcal{B}^{\prime}}^{\prime}$ can only be valid for the new $\mathrm{BN} \mathcal{B}^{\prime}$ if it encodes an ancestral relationship between $X_{\text {out }}$ and $X_{\mathrm{in}}$. Algorithm 2 modifies the structure of $\mathcal{E}_{\mathcal{B}}$ to meet the new restrictions. The resulting ET $\mathcal{E}_{\mathcal{B}^{\prime}}^{\prime}$ represents a complete subset of EOs $\mathcal{S}^{\prime} \subseteq \mathcal{S}$ (see Figure 7) that are also equivalent in $\mathcal{B}^{\prime}$.

Algorithm 2 receives a valid ET $\mathcal{E}_{\mathcal{B}}$, and arc addition $X_{\text {out }} \rightarrow X_{\text {in }}$. In $\mathcal{B}^{\prime}$, variable $X_{\text {out }}$ is added to the domain of $\phi_{X_{\text {in }}}$ (line 2). The clusters of the nodes that are predecessors of $\phi_{X_{\text {in }}}$ and descendants of $X_{\text {out }}$ contain $X_{\text {out }}$ after applying this change. There are three different scenarios that require performing different changes in the ET to ensure its validity.

If $X_{\text {out }}$ is a predecessor of $\phi_{X_{\text {in }}}$ in $\mathcal{E}_{\mathcal{B}^{\prime}}^{\prime}$, it is not necessary to make any changes in $\mathcal{E}_{\mathcal{B}^{\prime}}^{\prime}$. Otherwise, some nodes contain $X_{\text {out }}$ in their clusters but not in their predecessors, and $\mathcal{E}_{\mathcal{B}}^{\prime}$ is not sound. If $X_{f}=\operatorname{Pa}_{\mathcal{E}_{\mathcal{B}}}\left(\phi_{X_{\text {in }}}\right)$ is a predecessor of $X_{\text {out }}$ in $\mathcal{E}_{\mathcal{B}}$ (line 4 ), $X_{\text {out }}$ is set as the new parent of $\phi_{X_{\mathrm{in}}}$ in $\mathcal{E}_{\mathcal{B}^{\prime}}^{\prime}$ (line 5). Thus, $X_{\text {out }}$ is a predecessor of $\phi_{X_{\text {in }}}$ in $\mathcal{E}_{\mathcal{B}^{\prime}}^{\prime}$.

If $X_{\text {out }}$ is not a predecessor of $\phi_{X_{\text {in }}}$ in $\mathcal{E}_{\mathcal{B}}$ and $X_{f}$ is not a predecessor of $X_{\text {out }}$ in $\mathcal{E}_{\mathcal{B}}$ (line 6), the cluster of the node in $\left\{X_{\text {out }}\right\} \cup \operatorname{Pred}_{\mathcal{E}_{\mathfrak{B}}}\left(\phi_{X_{\text {in }}}\right) \cap \operatorname{Desc}_{\mathcal{E}_{\mathcal{B}}}\left(X_{\text {out }}\right)$ contains $X_{\text {out }}$ but the clusters of their predecessors in $\mathcal{E}_{\mathcal{B}}$ do not. Algorithm 2 


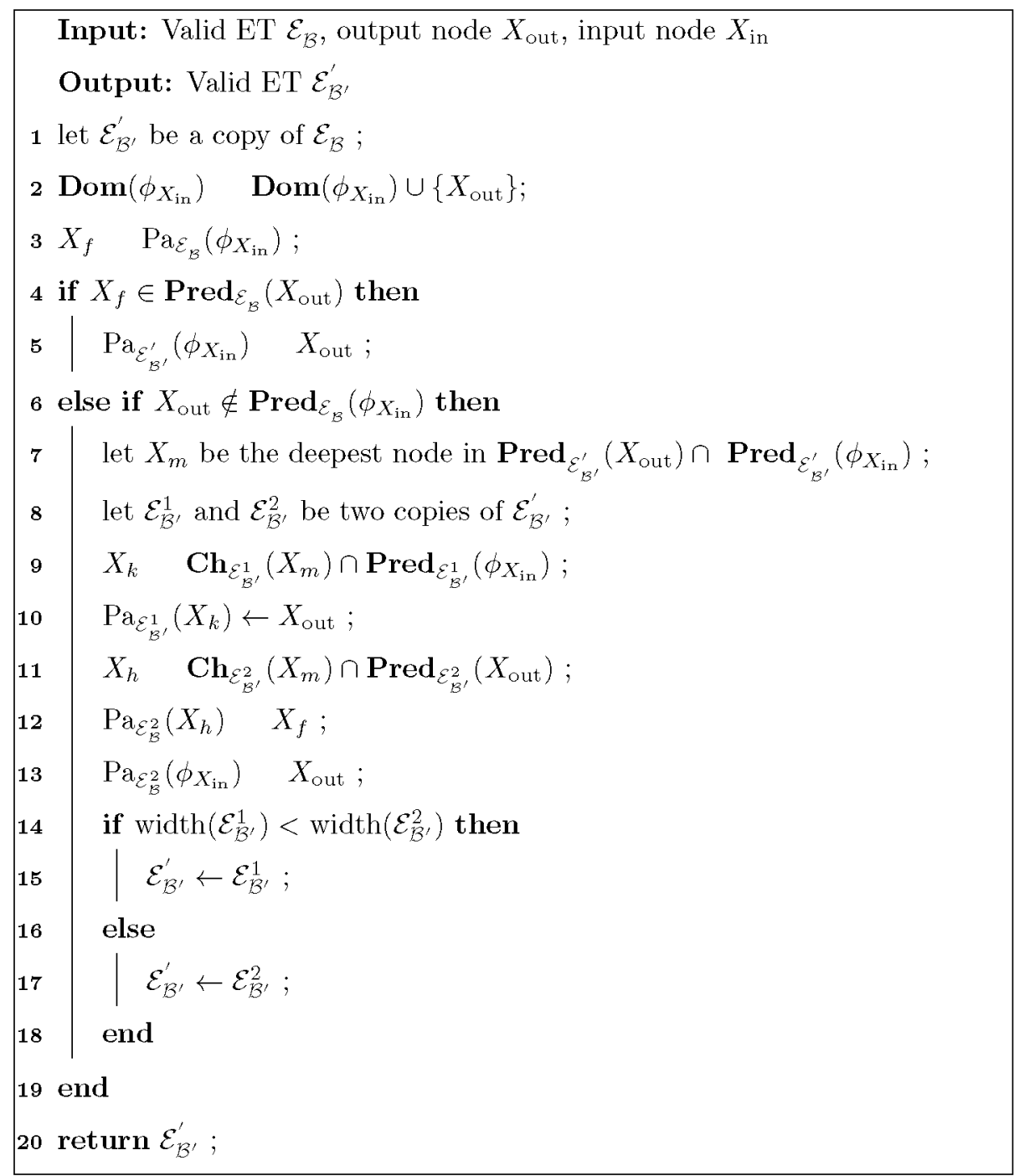

Algorithm 2: Compilation of the addition of arc $X_{\text {out }} \rightarrow X_{\text {in }}$ $\left(\operatorname{add}\left(\mathcal{E}_{\mathcal{B}}, X_{\text {out }}, X_{\text {in }}\right)\right)$ 


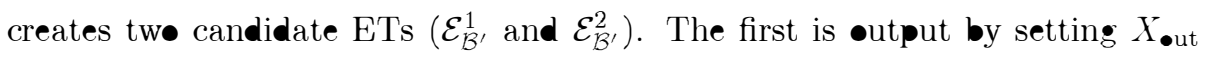
as the parent $\bullet X_{k}$ in $\mathcal{E}_{\mathcal{B}^{\prime}}^{1}$ (line 10), that is, the shalløwest predecesser $\bullet \boldsymbol{\phi}_{X_{\text {in }}}$

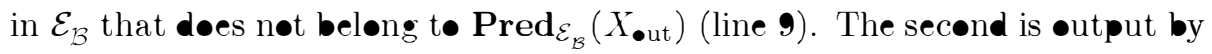
setting $\operatorname{Pa}_{\mathcal{E}_{\mathcal{B}}}\left(\boldsymbol{\phi}_{X_{\mathrm{in}}}\right)$ as the parent $\bullet X_{h}$ in $\mathcal{E}_{\mathcal{B}^{\prime}}^{2}$ (line 12), that is, the shalløwest

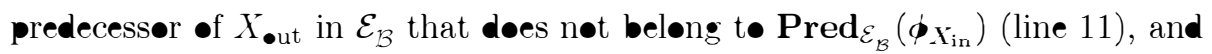
setting $X_{\bullet \text { ut }}$ as the new parent $\bullet \boldsymbol{\phi}_{X_{\text {in }}}$ in $\mathcal{E}_{\mathcal{B}^{\prime}}^{2}$ (line 13). $\mathcal{E}_{\mathcal{B}^{\prime}}^{\prime}$ is selected as the ET •f smaller width between $\mathcal{E}_{\mathcal{B}^{\prime}}^{1}$ and $\mathcal{E}_{\mathcal{B}^{\prime}}^{2}$ (lines $14-18$ ). Either way, $X_{\bullet \text { ut }}$ is a predecessør in $\mathcal{E}_{\mathcal{B}^{\prime}}^{\prime} \bullet$ f the n॰des in $\{X \bullet$ ut $\} \cup \operatorname{Pred}_{\mathcal{E}_{\mathcal{B}}}\left(\phi_{X_{\text {in }}}\right) \cap \operatorname{Desc}_{\mathcal{E}_{\mathcal{B}}}\left(X_{\bullet \text { ut }}\right)$, and the returned ET $\mathcal{E}_{\mathcal{B}^{\prime}}^{\prime}$ is valid (Lemma 1).

Lemma 1. Let $\mathcal{E}_{\mathcal{B}}$ be a valid ET that represents $\mathcal{B} \bullet$ ver $\mathcal{X}$. Given $X_{\bullet \text { ut }}, X_{\text {in }} \in$ $\mathcal{X}$, the ET $\mathcal{E}_{\mathcal{B}^{\prime}}^{\prime}$ yielded after applying add $\left(\mathcal{E}_{\mathcal{B}}, X_{\bullet}, X_{\text {in }}\right)$ in Alg॰rithm 2 , is als• a valid ET representing $\mathcal{B}^{\prime}$ over $\mathcal{X}$.

Prøof. See Appendix A.

This process can be performed efficiently in ETs •f bounded width (see Theorem 2).

Figure 8 shøws an example of the incremental compilation of the addition $\bullet$ arc $X_{1} \rightarrow X_{4}$ in $\mathcal{X}_{2}$. $\mathcal{E}_{\mathcal{B}}$ represents all the permutatiøns $\bullet\left\{X_{1}, X_{2}, X_{3}, X_{4}\right\}$ where $X_{2}$ and $X_{3}$ are eliminated before $X_{1}$. After adding $X_{1} \rightarrow X_{4}$ in $\mathcal{B}$, there are EOs represented by $\mathcal{E}_{\mathcal{B}}$ that are nøt equivalent for $\mathcal{B}^{\prime}$ (e.g., $\left\{X_{2}, X_{3}, X_{1}, X_{4}\right\}$ and $\left.\left\{X_{2}, X_{3}, X_{4}, X_{1}\right\}\right)$. Using Algørithm $2, X_{\bullet \text { ut }}=X_{1}$ and $X_{\text {in }}=X_{4}$. As

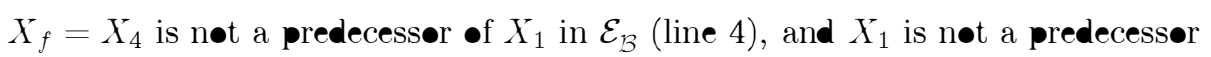
-f $\phi_{X_{4}}$ (line 6), tw• ETs $\mathcal{E}_{\mathcal{B}^{\prime}}^{1}$ and $\mathcal{E}_{\mathcal{B}^{\prime}}^{2}$ are created. Assuming that $\mathcal{E}_{\mathcal{B}^{\prime}}^{1}$ is smaller than $\mathcal{E}_{\mathcal{B}^{\prime}}^{2}, X_{1}$ is the new parent of $X_{4}$ in $\mathcal{E}_{\mathcal{B}^{\prime}}^{\prime}$.

\subsubsection{Are remøval}

The remøval $\bullet$ an $\operatorname{arc} X_{\bullet \text { ut }} \rightarrow X_{\text {in }}$ in $\mathcal{B}$ may compromise the cømpleteness -f an ET $\mathcal{E}_{\mathcal{B}}$. Let $\mathcal{S}$ be the set $\bullet$ EOs represented by $\mathcal{E}_{\mathcal{B}}$. The remøval of $X_{\bullet \text { ut }} \rightarrow X_{\text {in }}$ in $\mathcal{B}$ leads t• a reduction in the restrictiøns $\bullet$ EO equivalence in $\mathcal{S}$. This means that $\mathcal{E}_{\mathcal{B}}$ may nøt represent all the EOs that are equivalent t• EOs in $\mathcal{S}$. Algørithm 3 yields an ET that represents a cømplete superset $\bullet$ EOs 


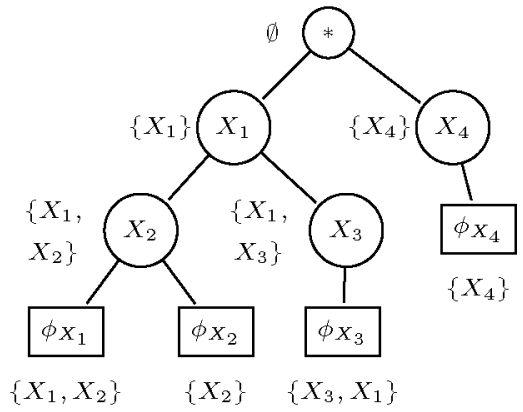

(a) $\mathcal{E}_{B}$

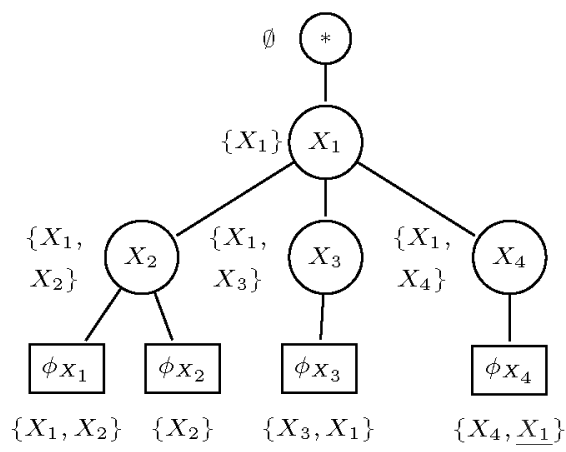

(b) $\mathcal{E}_{\mathcal{B}^{\prime}}^{\prime}$

Figure 8: (a) An ET $\mathcal{E}_{\mathcal{B}}$, and (b) the ET $\mathcal{E}_{\mathcal{B}}^{\prime}$, output after incrementally compiling the arc addition $X_{1} \rightarrow X_{4}$ (i.e., addition of $X_{1}$ to $\operatorname{Dom}\left(\phi_{X_{4}}\right)$ ) in $\mathcal{E}_{\mathcal{B}}$

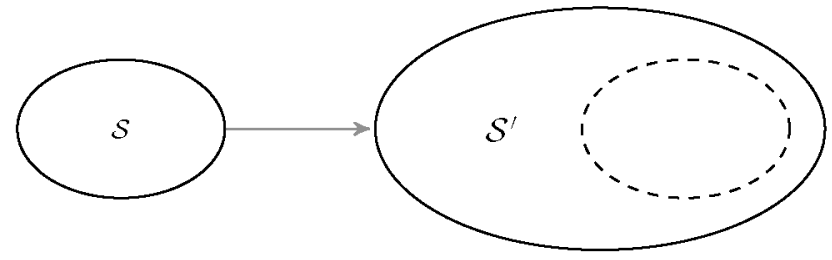

Figure 9: A set of EOs $\mathcal{S}$ that are equivalent for a BN $\mathcal{B}$, and a superset $\mathcal{S}^{\prime}$ of $\mathcal{S}$ that are equivalent for the $\mathrm{BN} \mathcal{B}^{\prime}$, yielded after removing an arc in $\mathcal{B}$

$\mathcal{S}^{\prime} \supseteq \mathcal{S}$ (see Figure 9) containing all the EOs that are equivalent to EOs in $\mathcal{S}$ for $\mathcal{B}^{\prime}$, which is the resulting $\mathrm{BN}$ after removing $\operatorname{arc} X_{\text {out }} \rightarrow X_{\text {in }}$ from $\mathcal{B}$.

After removing $X_{\text {out }} \rightarrow X_{\text {in }}$ from $\mathcal{B}$, the shallowest node in $\left(\operatorname{Pred}_{\mathcal{E}_{\mathcal{B}^{\prime}}^{\prime}}\left(\phi_{X_{\mathrm{in}}}\right) \cup\right.$ $\left.\left\{\phi_{X_{\text {in }}}\right\}\right) \cap \operatorname{Desc}_{\mathcal{E}_{\mathcal{B}^{\prime}}^{\prime}}\left(X_{\text {out }}\right)$, which we refer to as $X_{i}$ (line 4$)$, may not be complete. If $X_{i}$ is not complete (line 5), Algorithm 3 sets the deepest node in $\mathrm{Cls}_{\mathcal{E}_{\mathcal{B}^{\prime}}^{\prime}}\left(X_{i}\right)$, which we refer to as $X_{j}^{\prime}$, as its new parent (lines 6-7). Note that the idea behind this change is that the new parent of $X_{i}$ is in its cluster, making $X_{i}$ complete. After this change, the shallowest node in $\left\{X_{j}\right\} \cup \operatorname{Pred}_{\mathcal{E}_{\mathcal{B}^{\prime}}^{\prime}}\left(X_{j}\right) \cap \operatorname{Desc}_{\mathcal{E}_{\mathcal{B}^{\prime}}^{\prime}}\left(X_{j}^{\prime}\right)$ may not be sound. Thus, Algorithm 3 repeats the same process until every node is complete, guaranteeing the validity of every node in $\mathcal{E}_{\mathcal{B}^{\prime}}^{\prime}$ (Lemma 2).

Lemma 2. Let $\mathcal{E}_{\mathcal{B}}$ be a valid ET that represents $\mathcal{B}$ over $\mathcal{X}$. Given $X_{\text {out }}, X_{\text {in }} \in$ $\mathcal{X}$, the ET $\mathcal{E}_{\mathcal{B}^{\prime}}^{\prime}$ that represents $\mathcal{B}^{\prime}$ output after applying remove $\left(\mathcal{E}_{\mathcal{B}}, X_{\text {out }}, X_{\text {in }}\right)$ in 


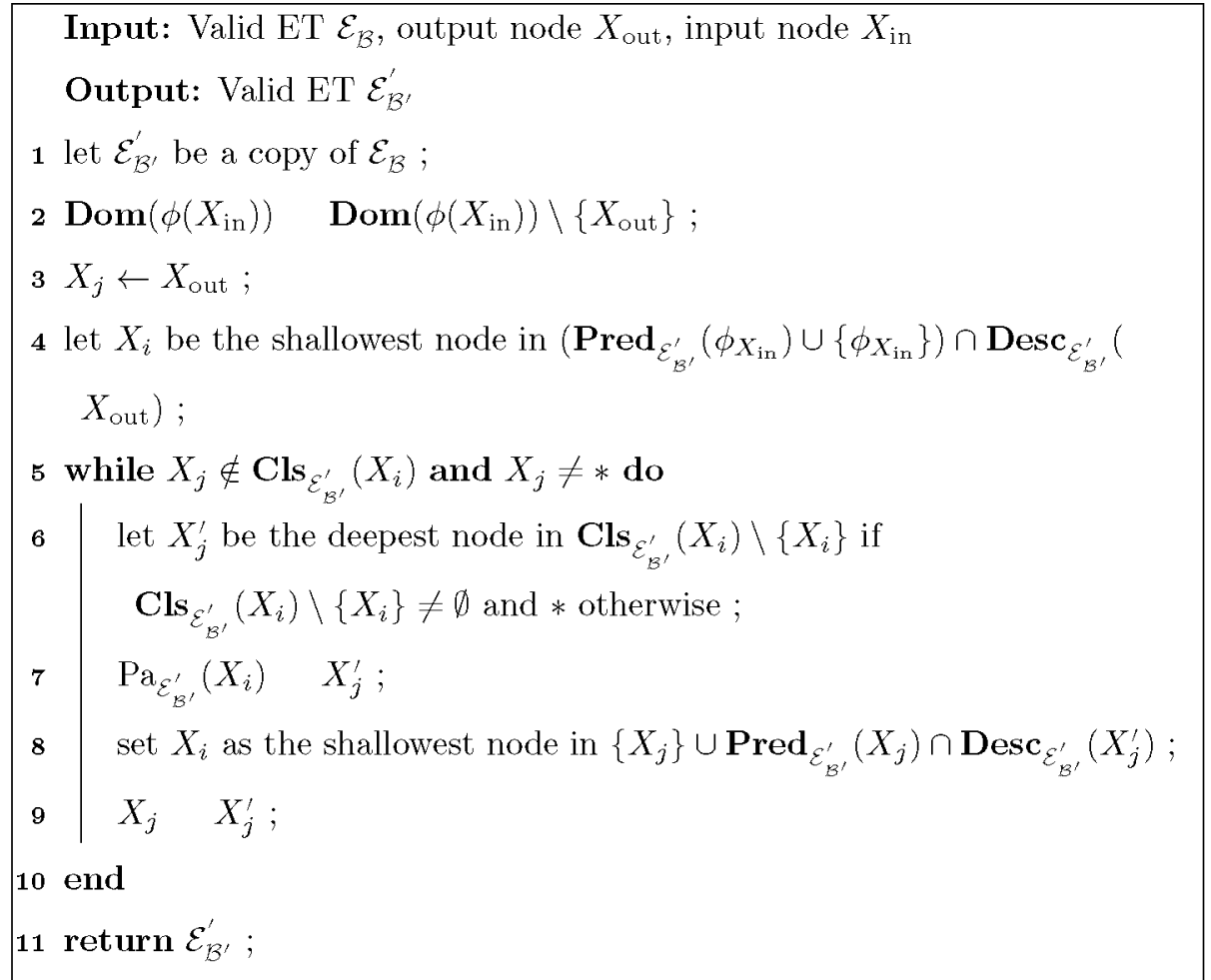

Algorithm 3: Compilation of the removal of arc $X_{\text {out }} \rightarrow X_{\text {in }}$ $\left(\operatorname{remove}\left(\mathcal{E}_{\mathcal{B}}, X_{\text {out }}, X_{\text {in }}\right)\right)$ 


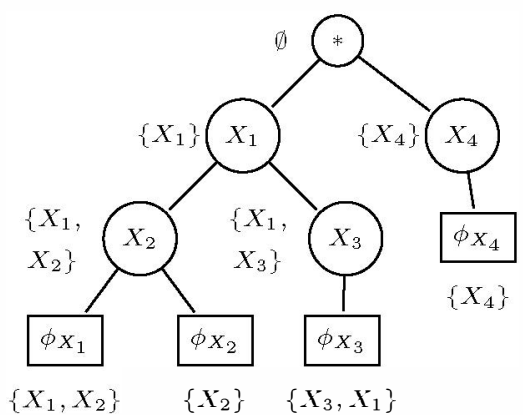

(a) $\mathcal{E}_{\mathcal{B}}$

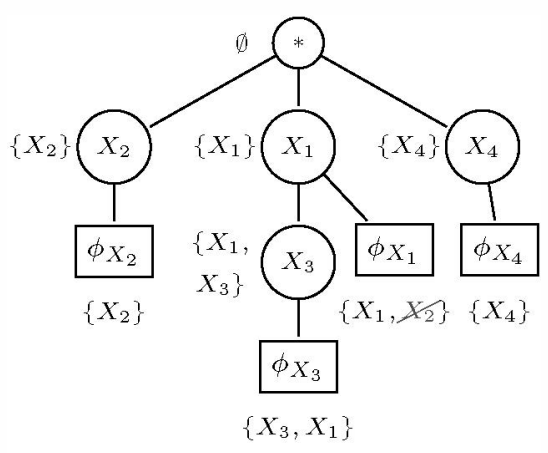

(b) $\mathcal{E}_{\mathcal{B}^{\prime}}^{\prime}$

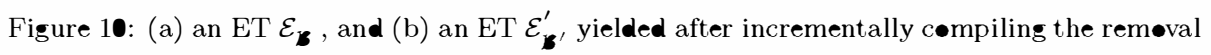

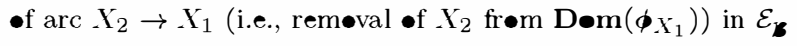

Algorithm 3, is als• valid.

Proof. See Appendix A.

Figure 10 shøws an example of høw Algørithm 3 cømpiles, in an ET $\mathcal{E}_{\mathcal{B}}$, the remøval of arc $X_{2} \rightarrow X_{1}$ in $\mathcal{B}$. Let $\mathcal{E}_{\mathcal{B}^{\prime}}^{\prime}$ be a copy of $\mathcal{E}_{\mathcal{B}}$ where $X_{2}$ has been removed frøm the domain of $\phi_{X_{1}}$. First, $X_{j}$ is set t• $X_{\bullet u t}$ (line 3 ). As $X_{\bullet \text { ut }}=X_{2}$ and $X_{\text {in }}=X_{1}, X_{i}$ is the shalløwest nøde in $\operatorname{Pred}_{\mathcal{E}_{\mathcal{B}}}\left(\phi_{X_{1}}\right) \cup\left\{X_{1}\right\}$ that is a descendant of $X_{2}$ (line 4), namely $\boldsymbol{\phi}_{X_{1}}$.

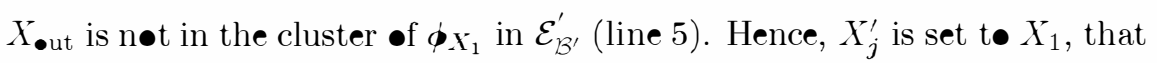
is, the deepest node in the cluster of $\boldsymbol{\phi}_{X_{1}}$ (line 6). $X_{1}$ is n॰w set as the parent

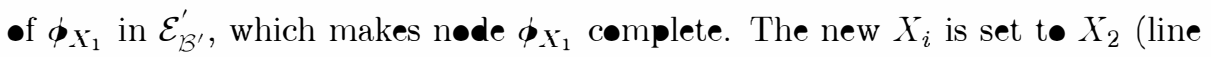
8), and the new $X_{j}$ is $X_{1}$ (line 9).

As $X_{1}$ is n॰t in the cluster of $X_{2}$ in $\mathcal{E}_{\mathcal{B}}$, the same process is applied again (lines 6-9). In this case, the root nøde $*$ is set as the parent of $X_{2}$ in $\mathcal{E}_{\mathcal{B}^{\prime}}^{\prime}$ and the new $X_{j}$ is $*$, ending the loop (line 5) and returning $\mathcal{E}_{\mathcal{B}^{\prime}}^{\prime}$.

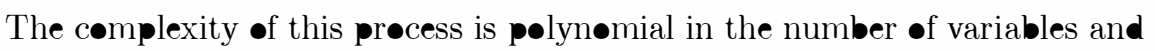
the width of the ET (see Theorem 2). 


\subsection{Optimization}

The methøds described above adapt an ET $\mathcal{E}_{\mathcal{B}}$ (resulting in $\mathcal{E}_{\mathcal{B}^{\prime}}^{\prime}$ ) t• a løcal

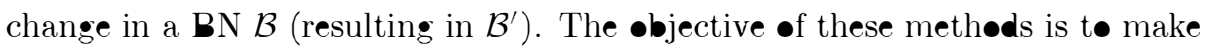

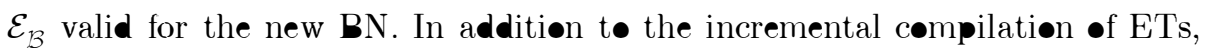

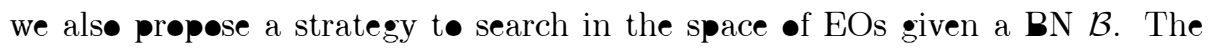

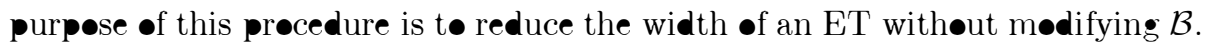
We use a simple and efficient heuristic tø address this prøblem. In this paper, we use the •ptimization process t• refine (reduce the width) •f the ETs returned by Algørithms 2-3.

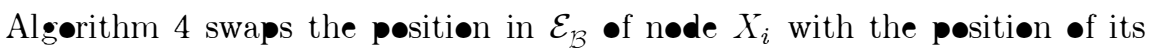
parent $X_{p}$, alsø changing the parents $\bullet$ any children $\bullet X_{i}$ in $\mathcal{E}_{\mathcal{B}}$ whøse validity is comprømised by the swap. Algorithm 4 guarantees the validity $\bullet$ the resulting

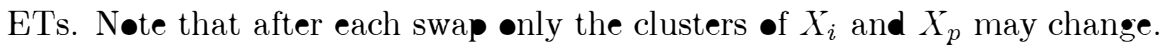

The sets $\bullet$ EOs represented by $\mathcal{E}_{\mathcal{B}}$ and the new ET $\mathcal{E}_{\mathcal{B}}^{\prime}$, which we refer t• as $\mathcal{S}$ and $\mathcal{S}^{\prime}$ respectively, are disjoint (see Figure 11). In $\mathcal{E}_{\mathcal{B}},\left(X_{p}<X_{i}\right)_{\imath}$ for any EO $\pi \in \mathcal{S}$, whereas $\left(X_{i}<X_{p}\right)_{\pi^{\prime}}$ in $\mathcal{E}_{\mathcal{B}}^{\prime}$ for any EO $\pi^{\prime} \in \mathcal{S}^{\prime}$.

Algørithm 4 prøceeds as folløws: First, a cøpy $\mathcal{E}_{\mathcal{B}}^{\prime} \bullet \mathcal{E}_{\mathcal{B}}$ is created (line 1), and $X_{j}$ is set t• the parent $\bullet X_{i}$ in $\mathcal{E}_{\mathcal{B}}$ (line 2). Then, the positions $\bullet X_{i}$ and $X_{j}$ are swapped in $\mathcal{E}_{\mathcal{B}}^{\prime}$ (lines 3 and 4 ). After that, the children $\bullet X_{i}$ whøse cluster contained $X_{j}$ set their parent in $\mathcal{E}_{\mathcal{B}}^{\prime}$ t• $X_{j}$ (lines 5-9), because •therwise these nodes would not be søund.

Lemma 3. Let $\mathcal{E}_{\mathcal{B}}$ be a valid ET that represents $\mathcal{B}$ over $\mathcal{X}$. Given $X_{i} \in \mathcal{X}$, the ET $\mathcal{E}_{\mathcal{B}}^{\prime}$ representing $\mathcal{B}^{\prime}$ yielded after applying swap $\left(\mathcal{E}_{\mathcal{B}}, X_{i}\right)$ in Algorithm 4 , is als•valid.

Prøof. See Appendix A.

Figure 12 shøws the result $\bullet$ applying Algørithm 4 t• an ET $\mathcal{E}_{\mathcal{B}}$. In this example, the positions $\bullet X_{3}$ and $X_{1}$ (parent $\bullet X_{3}$ in $\mathcal{E}_{\mathcal{B}}$ ) in the resulting ET $\mathcal{E}_{\mathcal{B}^{\prime}}$ are swapped. $\mathcal{E}_{\mathcal{B}}$ represents the EOs $\pi \bullet f\left\{X_{1}, X_{2}, X_{3}, X_{4}\right\}$ where $\left(X_{2}<\right.$ $\left.X_{1}\right)_{\pi}$ and $\left(X_{3}<X_{1}\right)_{\pi}\left(\mathbf{e . g} .,\left(X_{2}, X_{3}, X_{1}, X_{4}\right),\left(X_{4}, X_{3}, X_{2}, X_{1}\right), \ldots\right)$, while $\mathcal{E}_{\mathcal{B}^{\prime}}$ 


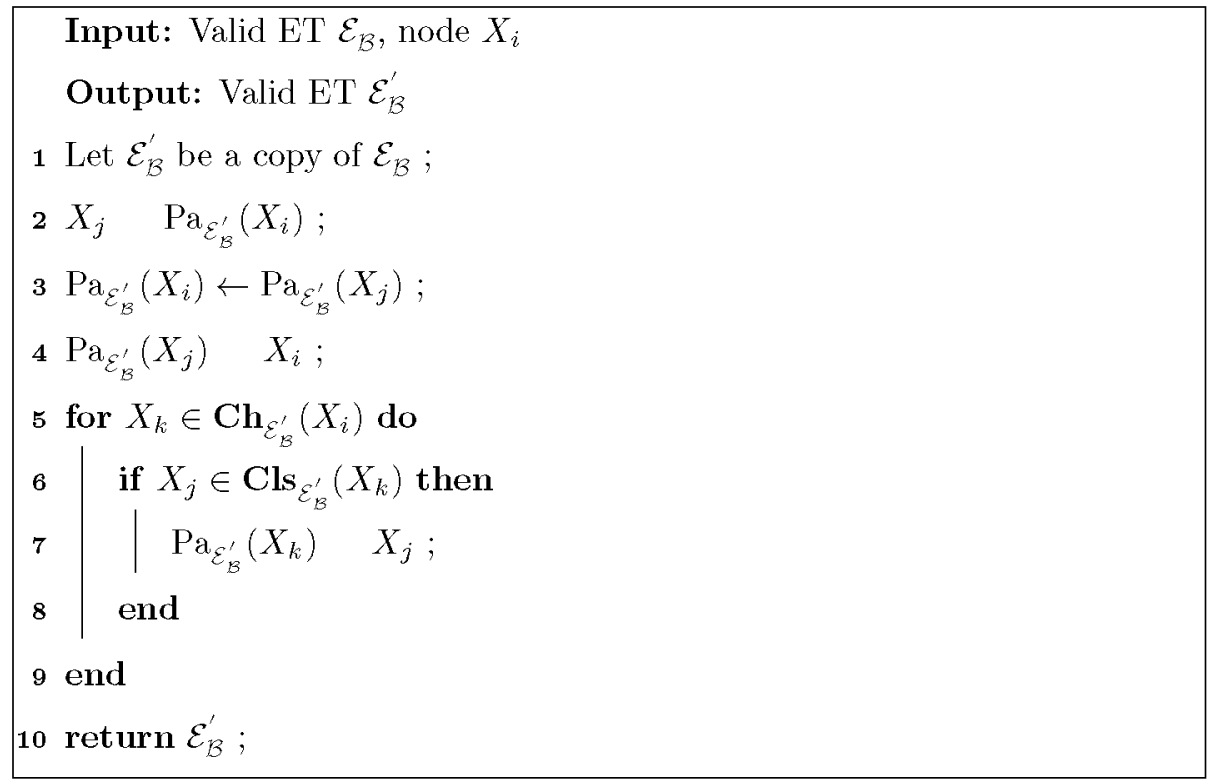

Algorithm 4: Swap of $X_{i}$ and $\operatorname{Pa}_{\mathcal{E}_{\mathcal{B}}}\left(X_{i}\right)$ in $\operatorname{ET} \mathcal{E}_{\mathcal{B}}\left(\operatorname{swap}\left(\mathcal{E}_{\mathcal{B}}, X_{i}\right)\right)$

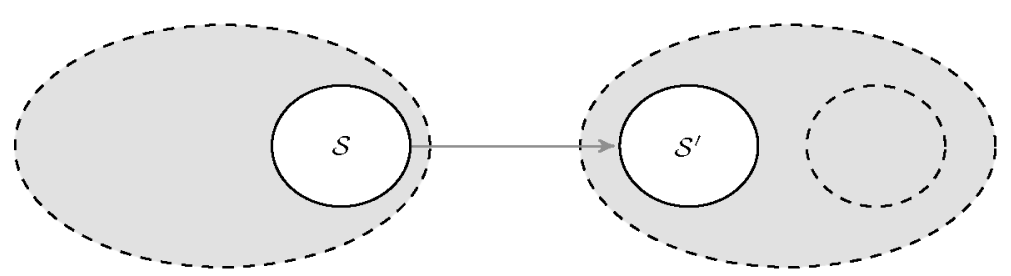

Figure 11: The EOs in $\mathcal{S}$ are equivalent for a $\mathrm{BN} \mathcal{B}$, and a swap produces other set of EOs $\mathcal{S}^{\prime}$, with $\mathcal{S} \cap \mathcal{S}^{\prime}=\emptyset$, where the EOs in $\mathcal{S}^{\prime}$ are also equivalent for $\mathcal{B}$ 


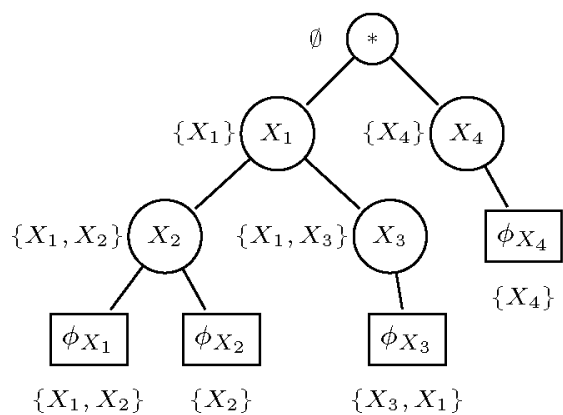

(a) $\mathcal{E}_{B}$

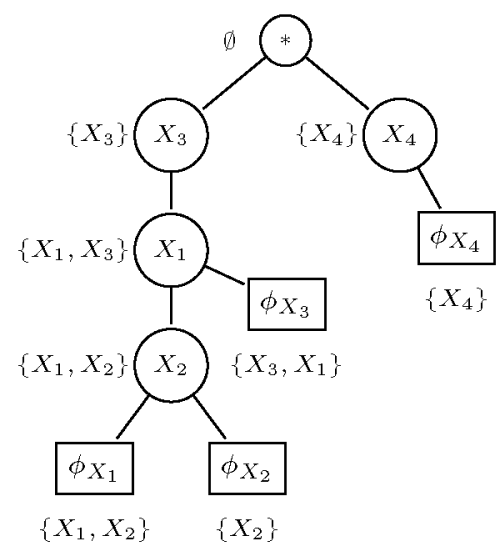

(b) $\mathcal{E}_{\mathcal{B}}^{\prime}$

Figure 12: (a) an $\operatorname{ET} \mathcal{E}_{\mathcal{B}}$, and (b) an ET $\mathcal{E}_{\mathcal{B}}^{\prime}$ yielded after swapping the positions of $X_{3}$ and $X_{1}$ in $\mathcal{E}_{\mathcal{B}}$

represents the EOs $\pi^{\prime}$ of $\left\{X_{1}, X_{2}, X_{3}, X_{4}\right\}$ where $\left(X_{2}<X_{1}\right)_{\pi^{\prime}}$ and $\left(X_{1}<X_{3}\right)_{\pi^{\prime}}$ (e.g., $\left.\left(X_{4}, X_{2}, X_{1}, X_{3}\right),\left(X_{2}, X_{4}, X_{1}, X_{3}\right), \ldots\right)$.

We use a greedy heuristic, which, given an $\mathrm{ET} \mathcal{E}_{\mathcal{B}}$ and a set of nodes for optimization $\left(\mathcal{X}_{\mathrm{opt}}\right)$, visits each node $X_{i} \in \mathcal{X}_{\mathrm{opt}}$ from the shallowest to the deepest, checking at each step whether swapping the position of $X_{i}$ and its parent reduces the width of the ET, see Algorithm 5.

Orderings that are good for one $\mathrm{BN} \mathcal{B}^{0}$ (i.e., their width is close to the treewidth of $\mathcal{B}^{0}$ ) may not be good for the $\mathrm{BN} \mathcal{B}$ yielded after applying a local change in $\mathcal{B}^{0}$. We perform the optimization process after the compilation of each local change. For efficiency, we select the nodes that may have a different cluster after compiling the local change to initialize $\mathcal{X}_{\text {opt }}$, given that they are more likely to produce relevant changes in the width of the ET. Next, we show the set of nodes selected for optimization $\left(\mathcal{X}_{\mathrm{opt}}\right)$ after compiling arc additions and removals. Each bullet point describes the assignment to $\mathcal{X}_{\text {opt }}$ at a possible scenario. We also explain the reason why the cluster of any of the nodes in $\mathcal{X}_{\text {opt }}$ may have changed.

- Addition of arc $X_{\text {out }} \rightarrow X_{\text {in }}$ : 


\section{Input: Valid ET $\mathcal{E}_{\mathcal{B}}$, set of nodes $\mathcal{X}_{\mathrm{opt}}$ \\ Output: Valid ET $\mathcal{E}_{\mathcal{B}}^{\prime}$}

1 Let $\mathcal{E}_{\mathcal{B}}^{\prime}$ be a copy of $\mathcal{E}_{\mathcal{B}}$;

2 Let $\boldsymbol{X}_{\text {opt }}$ be a list that contains the nodes in $\mathcal{X}_{\text {opt }}$ ordered from the shallowest to the deepest;

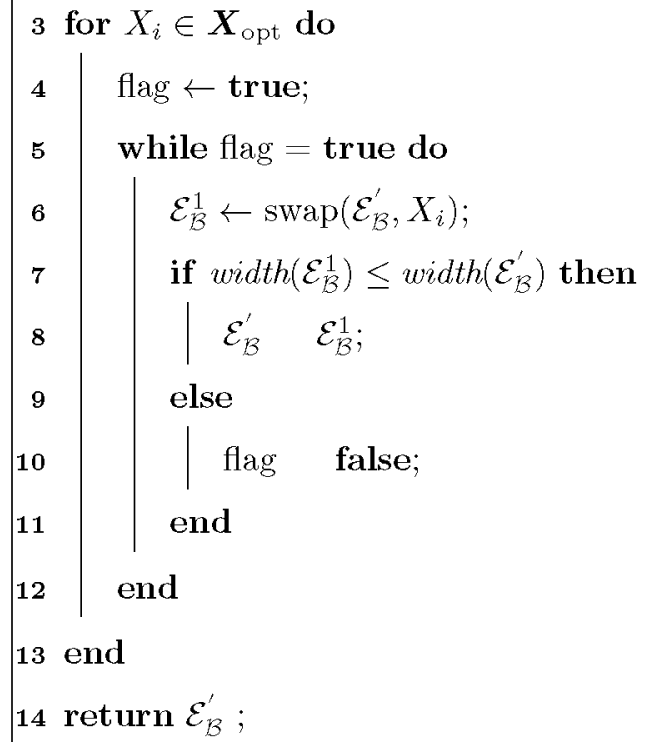

Algorithm 5: Optimization of an $\operatorname{ET}\left(\operatorname{optimize}\left(\mathcal{E}_{\mathcal{B}}, \mathcal{X}_{\mathrm{opt}}\right)\right)$ 
- If $X_{\text {out }} \in \operatorname{Pred}_{\mathcal{E}_{\mathcal{B}}}\left(\phi_{X_{\text {in }}}\right), \mathcal{X}_{\text {opt }}=\operatorname{Pred}_{\mathcal{E}_{\mathcal{B}}}\left(\phi_{X_{\text {in }}}\right) \cap \operatorname{Desc}_{\mathcal{E}_{B}}\left(X_{\text {out }}\right)$ :

The width of the clusters in $\operatorname{Pred}_{\mathcal{E}_{1}}\left(\phi_{X_{\text {in }}}\right) \cap \operatorname{Desc}_{\mathcal{E}_{\mathcal{B}}}\left(X_{\text {out }}\right)$ grows, given that they now contain $X_{\text {out }}$.

- Else, if $\operatorname{Pa}_{\mathcal{E}_{\mathcal{B}}}\left(\phi_{X_{\text {in }}}\right) \in \operatorname{Pred}_{\mathcal{E}_{\mathcal{B}}}\left(X_{\text {out }}\right), \mathcal{X}_{\text {opt }}=\left(\operatorname{Pred}_{\mathcal{E}_{\mathcal{B}}}\left(X_{\text {out }}\right) \cup\left\{X_{\text {out }}\right\}\right) \cap$

$\operatorname{Desc}_{\mathcal{E}_{\mathcal{B}}}\left(\mathrm{Pa}_{\mathcal{E}_{\mathcal{B}}}\left(\phi_{X_{\mathrm{in}}}\right)\right)$ :

The width of the clusters in $\left(\operatorname{Pred}_{\mathcal{E}_{\mathcal{B}}}\left(X_{\text {out }}\right) \cup\left\{X_{\text {out }}\right\}\right) \cap \operatorname{Desc}_{\mathcal{E}_{\mathcal{B}}}\left(\operatorname{Pa}_{\mathcal{E}_{\mathcal{B}}}(\right.$ $\left.\phi_{X_{\mathrm{in}}}\right)$ ) may grow, given that they now contain $\operatorname{Dom}\left(\phi_{X_{\mathrm{in}}}\right)$.

- Otherwise, $\mathcal{X}_{\text {opt }}=\left(\operatorname{Pred}_{\mathcal{E}_{\mathcal{B}}}\left(\phi_{X_{\text {in }}}\right) \cup \operatorname{Pred}_{\mathcal{E}_{\mathcal{B}}}\left(X_{\text {out }}\right) \cup\left\{X_{\text {out }}\right\}\right) \backslash\left(\operatorname{Pred}_{\mathcal{E}_{\mathcal{B}}}(\right.$ $\left.\left.\phi_{X_{\text {in }}}\right) \cap \operatorname{Pred}_{\mathcal{E}_{\mathcal{B}}}\left(X_{\text {out }}\right)\right)$ :

Node $\phi_{X_{\text {in }}}$ is set as a descendant of $X_{\text {out }}$, and the nodes in $\operatorname{Pred}_{\mathcal{E}_{\mathcal{B}}}($ $\left.\phi_{X_{\text {in }}}\right) \backslash\left(\operatorname{Pred}_{\varepsilon_{B}}\left(\phi_{X_{\text {in }}}\right) \cap \operatorname{Pred}_{\varepsilon_{B}}\left(X_{\text {out }}\right)\right)$ are either predecessors or descendants of $X_{\text {out }}$ and $\operatorname{Pred}_{\mathcal{E}_{\mathcal{B}}}\left(X_{\text {out }}\right) \backslash\left(\operatorname{Pred}_{\mathcal{E}_{\mathfrak{B}}}\left(\phi_{X_{\text {in }}}\right) \cap \operatorname{Pred}_{\mathcal{E}_{\mathcal{B}}}(\right.$ $\left.X_{\text {out }}\right)$ ) in the new ET.

- Removal of $\operatorname{arc} X_{\text {out }} \rightarrow X_{\text {in }}, \mathcal{X}_{\text {opt }}=\operatorname{Pred}_{\mathcal{E}_{\mathcal{B}}}\left(\phi_{X_{\text {in }}}\right) \cap \operatorname{Desc}_{\mathcal{E}_{\mathcal{B}}}\left(X_{j}\right)$ : Let $X_{j}$ be the last node that had a new child $X_{i}$ assigned by Algorithm 3. The nodes in $\operatorname{Pred}_{\mathcal{E}_{\mathcal{B}}}\left(\phi_{X_{\text {in }}}\right) \cap \operatorname{Desc}_{\mathcal{E}_{\mathcal{B}}}\left(X_{j}\right)$ may have smaller clusters in the new ET.

The optimization of an ET takes polynomial time in the number of variables and in the width of the ET (see Theorem 2).

\subsection{Learning elimination trees from data}

Using the incremental compilation and optimization methods described above, it is rather straightforward to learn ETs from a dataset $\mathcal{D}$ in combination with any score+search BN learning method that applies local changes during the search. Learning low inference complexity BNs with this approach is also easily derived. It can be achieved by bounding the width of each ET during the learning process (which bounds the treewidth of their corresponding BNs).

Theorem 1 ensures that any algorithm that uses the above strategy will always produce valid ETs. 
Theorem 1. Let $\mathcal{E}_{\mathcal{B}}$ be a valid ET $\bullet$ ver $\mathcal{X}$, and $\mathcal{E}_{\mathcal{B}}^{\prime}$ the result $\bullet$ incrementally compiling $\bullet n \mathcal{E}_{\mathcal{B}}$ any local change in $\mathcal{B}$ using Algorithms 2 and 3 and $\bullet$ ptimizing the resulting ET using Algorithm 5. Then $\mathcal{E}_{\mathcal{B}^{\prime}}^{\prime}$ is a valid ET.

Prøof. See Appendix A.

As we apply the incremental compilation and •ptimization methøds t• each candidate during the learning precess, efficiency is a critical issue. Theorem 2 bounds the computational time complexity of the incremental compilation and •ptimizatiøn methøds prøposed abøve.

Theorem 2. Let $\mathcal{E}_{\mathcal{B}}$ be a valid ET $\bullet$ ver a set $\bullet f$ variables $\mathcal{X}=\left\{X_{1}, \ldots, X_{n}\right\}$. The process described in Theorem 1 t॰ $\bullet$ utput $\mathcal{E}_{\mathcal{B}^{\prime}}^{\prime}$ can be performed in time $\boldsymbol{O}\left(n^{2} \cdot \operatorname{width}\left(\mathcal{E}_{\mathcal{B}}\right)\right)$.

Proof. See Appendix B.

Let $\mathcal{A}$ be any algørithm that learns the structure of a $\mathrm{BN}$ using only løcal changes in the structure of the network during the learning process. $\mathcal{A}$ can be adapted t• learn løw inference complexity BNs compiling and optimizing

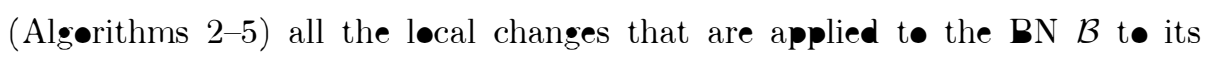
respective ET $\mathcal{E}_{\mathcal{B}}$. Thus, $\mathcal{E}_{\mathcal{B}}$ can be used t• bound the treewidth $\bullet \mathcal{B}$ using

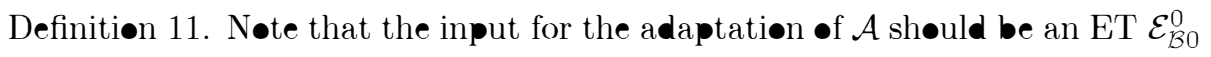
valid for the initial $\mathrm{BN} \mathcal{B}^{0}$.

\section{Experimental results}

In this sectiøn, we empirically analyzed the performance of the proposed

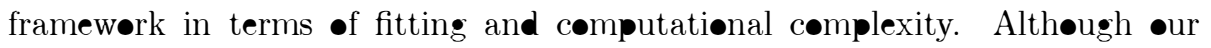
apprøach could be used with most score+search BN learning methøds, in the experiments we combine the incremental cømpilation and •ptimizatiøn methøds proposed in Section 4 with a greedy hill-climbing for the structure search. We call the resulting methød hc-ET. We compared hc-ET with k-greedy and k-

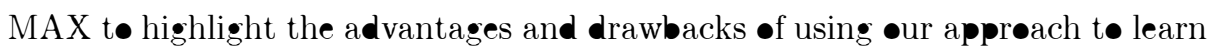


bounded treewidth BNs. We also tested a polynomial version of hc-ET that only considers arc additions during the structure search. We call this method hc-ET-poly.

To perform the experiments, we used 22 real-world datasets. These datasets were previously used in several papers $[62,63,64,53]$, and can be found at https://github.com/UCLA-StarAI/Density-Estimation-Datasets. Additionally, we generated synthetic data from 12 real-world BNs. These BNs were obtained from the bnlearn BN repository http://www. bnlearn. com/bnrepository/, and are cited therein. Table 1 briefly describes the basic properties of each dataset.

For each dataset we learned three BNs with each of the compared methods, using different treewidth bounds $(3,5$ and 7$)$. In all cases, the score function to maximize was BIC. k-greedy and k-MAX require to fix a maximum execution time, which we set to $n$ seconds (i.e., a second for each variable) to compute the cache of best parent sets and $n / 10$ seconds for the structure search. These values were used by Scanagatta et al. [53] in their experiments. To compare the results we used the following performance measures: the BIC score and the log-likelihood (LL) of the models in the training dataset, the learning time, and the treewidth of the returned models.

We analyzed the significance of the differences found for each performance measure in all the datasets and for all the treewidth bounds using the Friedman test with $\alpha=0.05$ and Holm's [65] and Shaffer's [66] post-hoc procedures. Both Holm's and Shaffer's procedures associate pairwise comparisons with a set of hypotheses and perform a step-down process with the corresponding set of ordered p-values to adjust the value of $\alpha[67]$.

Experiments were performed on a computer with an Intel Core i7-6700K CPU at $4.00 \mathrm{GHz}$ with $16 \mathrm{~GB}$ main memory, running Ubuntu 16.04 LTS. he-ET and he-ET-poly were written in Python 2.7.12 and $\mathrm{C}++11$ (version 5.4.0), while k-greedy and k-MAX were downloaded from http://ipg.idsia.ch/software/ blip and are written in Java. 
Table 1: Basic properties of the datasets
(a) Real-world datasets
(b) Synthetic datasets

\begin{tabular}{|c|c|c|c|c|c|}
\hline Dataset & N. vars & $\mathrm{N}$. inst & Dataset & N. vars & N. inst \\
\hline NLTCS & 16 & 16,181 & Hailfinder & 56 & 5,000 \\
\hline MSNBC1 & 17 & 291,326 & Hepar II & 70 & 5,000 \\
\hline KDDCup & 65 & 180,092 & Win95pts & 76 & 5,000 \\
\hline Plants & 69 & 17,412 & Pathfinder & 135 & 5,000 \\
\hline Audio & 100 & 15,000 & Munin1 & 186 & 5,000 \\
\hline Jester & 100 & 9,000 & Andes & 223 & 5,000 \\
\hline Netflix & 100 & 15,000 & Diabetes & 413 & 5,000 \\
\hline Accidents & 111 & 12,758 & Pigs & 413 & 5,000 \\
\hline Retail & 135 & 22,041 & Link & 724 & 5,000 \\
\hline Pumsb-star & 163 & 12,262 & Munin2 & 1,003 & 5,000 \\
\hline DNA & 180 & 1,600 & Munin3 & 1,041 & 5,000 \\
\hline Kosarek & 190 & 33,375 & Munin4 & 1,038 & 5,000 \\
\hline MSWeb & 294 & 29,441 & & & \\
\hline Book & 500 & 8,700 & & & \\
\hline EachMovie & 500 & 4,525 & & & \\
\hline WebKB & 839 & 2,803 & & & \\
\hline Reuters-521 & 889 & 6,532 & & & \\
\hline 20 NewsGroup & 910 & 11,293 & & & \\
\hline Movie reviews & 1,001 & 1,600 & & & \\
\hline $\mathrm{BBC}$ & 1,058 & 1,670 & & & \\
\hline Voting & 1,359 & 1,214 & & & \\
\hline Ad & 1,556 & 2,461 & & & \\
\hline
\end{tabular}


Table 2: Comparison of the methods in all the datasets, using a treewidth bound of 3 . The optimal results are denoted in boldface.

\begin{tabular}{|c|c|c|c|c|}
\cline { 2 - 5 } \multicolumn{1}{c|}{} & hc-ET & hc-ET-poly & k-greedy & k-MAX \\
\hline mean rank BIC & $\mathbf{1 . 3 2} \pm \mathbf{0 . 5 3}$ & $1.79 \pm 0.54$ & $3.79 \pm 0.41$ & $3.09 \pm 0.62$ \\
mean rank LL & $\mathbf{1 . 3 8} \pm \mathbf{0 . 5 5}$ & $1.68 \pm 0.47$ & $3.79 \pm 0.41$ & $3.15 \pm 0.56$ \\
mean rank time & $1.88 \pm 0.33$ & $\mathbf{1 . 1 2} \pm 0.33$ & $3.56 \pm 0.5$ & $3.44 \pm 0.5$ \\
mean rank treewidth & $3 \pm 0$ & $3 \pm 0$ & $2.85 \pm 0.66$ & $2.68 \pm 0.77$ \\
\hline
\end{tabular}

Table 3: Comparison of the methods in all the datasets, using a treewidth bound of 5 . The optimal results are denoted in boldface.

\begin{tabular}{|c|c|c|c|c|}
\cline { 2 - 5 } \multicolumn{1}{c|}{} & \multicolumn{1}{c|}{ hc-ET } & hc-ET-poly & k-greedy & k-MAX \\
\hline mean rank BIC & $\mathbf{1 . 3 5} \pm \mathbf{0 . 6 9}$ & $1.91 \pm 0.57$ & $3.76 \pm 0.61$ & $2.97 \pm 0.63$ \\
mean rank LL & $\mathbf{1 . 4 7} \pm \mathbf{0 . 7 1}$ & $1.71 \pm 0.52$ & $3.71 \pm 0.63$ & $3.12 \pm 0.59$ \\
mean rank time & $1.88 \pm 0.33$ & $\mathbf{1 . 1 2} \pm \mathbf{0 . 3 3}$ & $3.56 \pm 0.5$ & $3.44 \pm 0.5$ \\
mean rank treewidth & $4.88 \pm 0.48$ & $4.91 \pm 0.38$ & $3.56 \pm 1.13$ & $3.41 \pm 1.08$ \\
\hline
\end{tabular}

\subsection{Comparison}

Tables 2-4 give an overview of the results obtained using the treewidth bounds 3,5 and 7 , respectively. For each performance measure, the mean rank \pm the standard deviation of each method over all the datasets is shown. The ranking of the methods is given by their average performance (BIC, LL and time) compared to the rest (i.e., the best is ranked the first and the worst is ranked the fourth). The mean treewidth \pm the standard deviation is also shown. The detailed results are supplied as Supplementary Material.

Figures 13-15 graphically present the results obtained with Holm's and Shaffer's procedure for each performance measure in all datasets. In the figures, groups of methods that are not significantly different are connected with a thick horizontal line. We used the graphical representation proposed by Demšar [68]. Each figure represents both procedures, given that the significant differences observed by Shaffer's procedure are identical to those observed by Holm's procedure. 
Table 4: Comparison of the methods in all the datasets, using a treewidth bound of 7 . The optimal results are denoted in boldface.

\begin{tabular}{|c|c|c|c|c|}
\cline { 2 - 5 } \multicolumn{1}{c|}{} & hc-ET & hc-ET-poly & k-greedy & k-MAX \\
\hline mean rank BIC & $\mathbf{1 . 3 5} \pm \mathbf{0 . 6 9}$ & $1.97 \pm 0.58$ & $3.79 \pm 0.59$ & $2.88 \pm 0.69$ \\
mean rank LL & $\mathbf{1 . 2 9} \pm \mathbf{0 . 4 6}$ & $1.82 \pm 0.52$ & $3.74 \pm 0.62$ & $3.15 \pm 0.56$ \\
mean rank time & $1.91 \pm 0.29$ & $\mathbf{1 . 0 9} \pm \mathbf{0 . 2 9}$ & $3.41 \pm 0.5$ & $3.59 \pm 0.5$ \\
mean rank treewidth & $6.74 \pm 0.96$ & $6.74 \pm 0.86$ & $3.91 \pm 1.31$ & $4 \pm 1.44$ \\
\hline
\end{tabular}

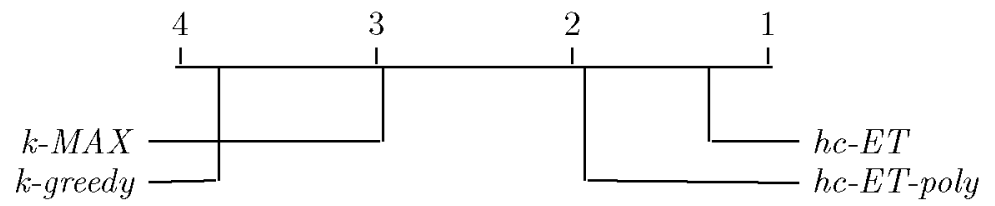

Figure 13: Comparison of BIC scores with Holm's and Shaffer's tests.

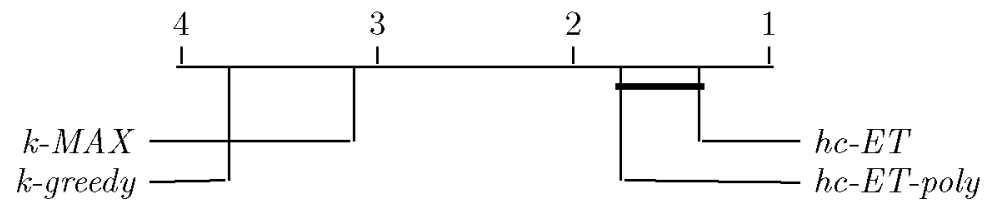

Figure 14: Comparison of log-likelihood with Holm's and Shaffer's tests.

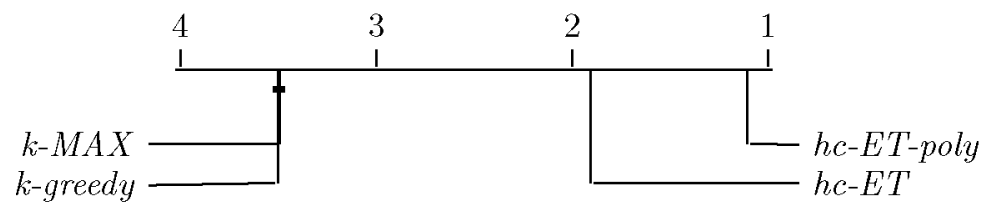

Figure 15: Comparison of learning time with Holm's and Shaffer's tests. 
Figure 13 shows significant differences between the BIC score achieved by all the methods; hc-ET performs the best overall, followed by hc-ET-poly, k-MAX, and k-greedy. Moreover, Tables 2-4 show that similar results can be found for all the tested treewidth bounds. The detailed results also show that he-ET performs better than k-MAX and k-greedy in over $94 \%$ of the experiments, and performs better than hc-ET-poly in around $80 \%$ of the experiments. The treewidth of the models output by each method suggests that one of the reasons why our proposal manages to optimize better the BIC score is that it allows a tighter fitting to the treewidth bound.

The comparison of the log-likelihood in Tables 2-4 and Figure 14 leads us to conclusions that are similar to those drawn for the BIC score. Nevertheless, in this case no significant differences were found between he-ET and he-ET-poly. This suggests that hc-ET-poly requires a higher number of parameters to obtain a similar fitting to he-ET in terms of log-likelihood.

As shown in Figure 15, we observed significant differences between the learning time of all the methods with the exception of k-MAX and k-greedy. The latter result was expected, given that the imposed limit in execution time of both approaches is the same. hc-ET-poly is the fastest method in all cases, followed by hc-et. However, we must be cautious when interpreting these results. First, these methods are implemented in different programming languages. Second, the bound in execution time set for k-greedy and k-MAX compels their learning time to scale linearly. Therefore, the difference in learning time between our method and k-MAX and k-greedy is clearly higher in the smaller datasets. Finally, although hc-et takes slightly more time than hc-ET-poly in all the experiments, we think that the improvement in BIC score is worthwhile in most situations.

\section{Conclusions and future research}

Traditional methods for learning BNs usually output models where exact inference is intractable. In this paper, we provide a novel framework for learning 
tractable BNs. We defined valid ETs, and proposed compilation methods for adapting valid ETs to any local change that may be applied to a BN (i.e., arc addition, removal, and reversal). We proved that the proposed methods always return valid ETs in polynomial time (Theorems 1 and 2). Our approach can be easily combined with any score+search BN learning method that uses only local changes in the network during the structure search. Valid ETs can be used to search in the combined space of DAGs and EOs, avoiding redundant solutions (i.e., all the EOs that are equivalent for a BN are represented by the same valid ET). Hence, we used this representation to efficiently bound the inference complexity of each BN during the learning process.

Experimental results showed that our approach places a tight upper bound on the inference complexity of the networks. The models learned with the proposed methods were competitive with other state-of-the-art methods, performing better in terms of BIC score and log-likelihood in most cases.

Future research will focus on adapting this framework to learning tractable multidimensional BN classifiers (MBCs) $[69,70]$. Most probable explanations can be computed in polynomial time in the treewidth of the pruned graph of an MBC [71], that is, a transformation of its structure that entails moralizing the respective DAG and removing the feature variables from the resultant graph. Thus, the application of the methods proposed in this paper to bound the treewidth of the pruned graph of an MBC should lead to tractable MBCs.

Also, we aim to study the relationship between the density of DAGs and the number of equivalent EOs. This would clarify the situations in which it is better to use ETs during the learning process.

Existing methods for learning BNs from incomplete datasets, such as the structural expectation-maximization algorithm [72], require inference during the learning process. We plan to bound the inference complexity of the models to make the structure search tractable when there are missing values or latent variables. Scanagatta et al. [53] successfully introduced the k-MAX algorithm in the maximization step of the structural expectation-maximization algorithm. Thus, we think that adapting our proposal to this problem could lead to promising 
results.

\section{Acknowledgments}

This work has been partially supported by the Spanish Ministry of Economy and Competitiveness through the Cajal Blue Brain (C080020-09; the Spanish partner of the Blue Brain initiative from EPFL) and TIN2016-79684-P projects, by the Regional Government of Madrid through the S2013/ICE-2845-CASICAM-CM project, and by Fundación BBVA grants to Scientific Research Teams in Big Data 2016. This project has received funding from the European Union's Horizon 2020 Framework Programme for Research and Innovation under Specific Grant Agreement No. 785907 (HBP SGA2). M. Benjumeda is supported by a

predoctoral contract for the formation of doctors from the Spanish Ministry of Economy and Competitiveness (BES-2014-068637).

\section{References}

[1] J. Pearl, Probabilistic Reasoning in Intelligent Systems: Networks of Plausible Inference, Morgan Kaufmann, 1988.

[2] D. Koller, N. Friedman, Probabilistic Graphical Models: Principles and Techniques - Adaptive Computation and Machine Learning, The MIT Press, 2009.

[3] C. Bielza, P. Larrañaga, Discrete Bayesian network classifiers: A survey, ACM Computing Surveys 47 (1) (2014) Article 5.

[4] J. M. Peña, J. A. Lozano, P. Larrañaga, Learning Bayesian networks for clustering by means of constructive induction, Pattern Recognition Letters 20 (1999) 1219-1230.

[5] D. T. Pham, G. A. Ruz, Unsupervised training of Bayesian networks for data clustering, in: Proceedings of the Royal Society of London A: Mathematical, Physical and Engineering Sciences, Vol. 465, The Royal Society, 2009, pp. 2927-2948. 
[6] D. Heckerman, D. Geiger, D. M. Chickering, Learning Bayesian networks: The combination of knowledge and statistical data, Machine Learning 20 (3) (1995) 197-243.

[7] G. F. Cooper, E. Herskovits, A Bayesian method for constructing Bayesian belief networks from databases, in: Proceedings of the 7th Conference on Uncertainty in Artificial Intelligence, Morgan Kaufmann Publishers Inc., 1991, pp. 86-94.

[8] G. F. Cooper, E. Herskovits, A Bayesian method for the induction of probabilistic networks from data, Machine Learning 9 (4) (1992) 309-347.

[9] H. Akaike, A new look at the statistical model identification, IEEE Transactions on Automatic Control 19 (6) (1974) 716-723.

[10] G. Schwarz, Estimating the dimension of a model, The Annals of Statistics $6(2)(1978) 461-464$.

[11] R. R. Bouckaert, Probabilistic network construction using the minimum description length principle, in: Symbolic and Quantitative Approaches to Reasoning and Uncertainty, Vol. 747, Springer, 1993, pp. 41-48.

[12] W. Lam, F. Bacchus, Learning Bayesian belief networks: An approach based on the MDL principle, Computational Intelligence 10 (3) (1994) $269-$ 293.

[13] S. Arnborg, D. G. Corneil, A. Proskurowski, Complexity of finding embeddings in a k-tree, SIAM Journal on Algebraic Discrete Methods 8 (2) (1987) 277-284.

[14] K. Grant, M. C. Horsch, Methods for constructing balanced elimination trees and other recursive decompositions, International Journal of Approximate Reasoning 50 (9) (2009) 1416-1424.

[15] K. Shoikhet, D. Geiger, A practical algorithm for finding optimal triangulations, in: Proceedings of the 14th National Conference on Artificial 
Intelligence and Ninth Conference on Innovative Applications of Artificial Intelligence, AAAI Press, 1997, pp. 185-190.

[16] F. V. Fomin, Y. Villanger, Treewidth computation and extremal combinatorics, Combinatorica 32 (3) (2012) 289-308.

[17] F. V. Fomin, D. Kratsch, I. Todinca, Exact (exponential) algorithms for treewidth and minimum fill-in, in: Automata, Languages and Programming, Vol. 3142, Springer, 2004, pp. 568-580.

[18] H. L. Bodlaender, F. V. Fomin, A. M. Koster, D. Kratsch, D. M. Thilikos, On Exact Algorithms for Treewidth, Springer, 2006.

[19] H. M. Markowitz, The elimination form of the inverse and its application to linear programming, Management Science 3 (3) (1957) 255-269.

[20] U. B. Kjærulff, Triangulation of graphs-algorithms giving small total state space, Tech. rep. (1990).

[21] D. J. Rose, R. E. Tarjan, G. S. Lueker, Algorithmic aspects of vertex elimination on graphs, SIAM Journal on Computing 5 (2) (1976) 266-283.

[22] R. E. Tarjan, M. Yannakakis, Simple linear-time algorithms to test chordality of graphs, test acyclicity of hypergraphs, and selectively reduce acyclic hypergraphs, SIAM Journal on Computing 13 (3) (1984) 566-579.

[23] A. Berry, J. R. Blair, P. Heggernes, B. W. Peyton, Maximum cardinality search for computing minimal triangulations of graphs, Algorithmica 39 (4) (2004) $287-298$.

[24] U. Kjærulff, Optimal decomposition of probabilistic networks by simulated annealing, Statistics and Computing 2 (1) (1992) 7-17.

[25] P. Larrañaga, C. M. Kuijpers, M. Poza, R. H. Murga, Decomposing Bayesian networks: Triangulation of the moral graph with genetic algorithms, Statistics and Computing 7 (1) (1997) 19-34. 
[26] F. Clautiaux, A. Moukrim, S. Nègre, J. Carlier, Heuristic and metaheuristic methods for computing graph treewidth, RAIRO-Operations ResearchRecherche Opérationnelle 38 (1) (2004) 13-26.

[27] A. Koster, Frequency assignment: Models and algorithms, Ph.D. thesis, Maastricht University (1999).

[28] H. L. Bodlaender, A. M. Koster, Treewidth computations I. Upper bounds, Information and Computation 208 (3) (2010) 259-275.

[29] H. L. Bodlaender, A linear time algorithm for finding tree-decompositions of small treewidth, in: Proceedings of the 25th Annual ACM Symposium on Theory of Computing, ACM, 1993, pp. 226-234.

[30] J. Kwisthout, Most probable explanations in Bayesian networks: Complexity and tractability, International Journal of Approximate Reasoning $52(9)$ (2011) $1452-1469$.

[31] G. F. Cooper, The computational complexity of probabilistic inference using Bayesian belief networks, Artificial Intelligence 42 (2) (1990) 393-405.

[32] P. Dagum, M. Luby, Approximating probabilistic inference in Bayesian belief networks is NP-hard, Artificial Intelligence 60 (1) (1993) 141-153.

[33] J. Pearl, Reverend Bayes on inference engines: A distributed hierarchical approach, in: Proceedings of the 2nd AAAI Conference on Artificial Intelligence, AAAI Press, 1982, pp. 133-136.

[34] J. H. Kim, J. Pearl, A computational model for causal and diagnostic reasoning in inference systems, in: Proceedings of the 8th International Joint Conference on Artificial Intelligence, Vol. 1, Morgan Kaufmann Publishers Inc., 1983, pp. 190-193.

[35] R. D. Shachter, Evidence absorption and propagation through evidence reversals, in: Proceedings of the 5th Annual Conference on Uncertainty in Artificial Intelligence, North-Holland Publishing Co., 1990, pp. 173-190. 
[36] N. L. Zhang, D. Poole, A simple approach to Bayesian network computations, in: Proceedings of the 10th Canadian Conference on Artificial Intelligence, 1994, pp. 171-178.

[37] J. Pearl, A constraint propagation approach to probabilistic reasoning, in: Proceedings of the 1st Annual Conference on Uncertainty in Artificial Intelligence, 1985, pp. 357-369.

[38] A. Darwiche, Recursive conditioning, Artificial Intelligence 126 (1) (2001) $5-41$.

[39] P. P. Shenoy, G. Shafer, Axioms for probability and belief-function propagation, in: Proceedings of the 4th Annual Conference on Uncertainty in Artificial Intelligence, North-Holland Publishing Co., 1990, pp. 169-198.

[40] F. Jensen, S. Lauritzen, K. Olsen, Bayesian updating in recursive graphical models by local computation, Computational Statistics Quarterly 4 (1990) $269-282$.

[41] C. Boutilier, N. Friedman, M. Goldszmidt, D. Koller, Context-specific independence in Bayesian networks, in: Proceedings of the 12th International Conference on Uncertainty in Artificial Intelligence, Morgan Kaufmann Publishers Inc., 1996, pp. 115-123.

[42] A. Darwiche, A differential approach to inference in Bayesian networks, Journal of the ACM 50 (3) (2003) 280-305.

[43] M. Niepert, G. van den Broeck, Tractability through exchangeability: A new perspective on efficient probabilistic inference, in: Proceedings of the 28th AAAI Conference on Artificial Intelligence, AAAI Press, 2014, pp. $2467-2475$.

[44] J. Korhonen, P. Parviainen, Exact learning of bounded tree-width Bayesian networks, in: Proceedings of the 16th International Conference on Artificial Intelligence and Statistics, Vol. 31, Proceedings of Machine Learning Research, 2013, pp. 370-378. 
[45] J. Berg, M. Järvisalo, B. Malone, Learning optimal bounded treewidth Bayesian networks via maximum satisfiability, in: Proceedings of the 17th International Conference on Artificial Intelligence and Statistics, Vol. 33, 2014, pp. 86-95.

[46] S. Nie, D. D. Mauá, C. P. de Campos, Q. Ji, Advances in learning Bayesian networks of bounded treewidth, in: Advances in Neural Information Processing Systems, 2014, pp. 2285-2293.

[47] P. Parviainen, H. Shahrabi Farahani, J. Lagergren, Learning bounded treewidth Bayesian networks using integer linear programming, in: Proceedings of the 17th International Conference on Artificial Intelligence and Statistics, Vol. 33, Proceedings of Machine Learning Research, 2014, pp. 751-759.

[48] G. Elidan, S. Gould, Learning bounded treewidth Bayesian networks, in: Advances in Neural Information Processing Systems, 2009, pp. 417-424.

[49] S. Nie, C. P. de Campos, Q. Ji, Efficient learning of Bayesian networks with bounded tree-width, International Journal of Approximate Reasoning 80 (2017) 412-427.

[50] M. Scanagatta, G. Corani, C. P. de Campos, M. Zaffalon, Learning treewidth-bounded Bayesian networks with thousands of variables, in: Proceedings of the 30th International Conference on Neural Information Processing Systems, 2016, pp. 1470-1478.

[51] M. Scanagatta, C. P. d. Campos, G. Corani, M. Zaffalon, Learning Bayesian networks with thousands of variables, in: Proceedings of the 28th International Conference on Neural Information Processing Systems, MIT Press, 2015, pp. 1864-1872.

[52] J. Cussens, Bayesian network learning with cutting planes, in: Proceedings of the Twenty-Seventh Conference on Uncertainty in Artificial Intelligence, AUAI Press, 2011, pp. 153-160. 
[53] M. Scanagatta, G. Corani, M. Zaffalon, J. Yoo, U. Kang, Efficient learning of bounded-treewidth Bayesian networks from complete and incomplete data sets, International Journal of Approximate Reasoning 95 (2018) 152166.

[54] F. R. Bach, M. I. Jordan, Thin junction trees, in: Advances in Neural Information Processing Systems, 2001, pp. 569-576.

[55] D. Karger, N. Srebro, Learning Markov networks: Maximum bounded treewidth graphs, in: Proceedings of the 12th Annual ACM-SIAM Symposium on Discrete Algorithms, Society for Industrial and Applied Mathematics, 2001, pp. 392-401.

[56] A. Chechetka, C. Guestrin, Efficient principled learning of thin junction trees, in: Advances in Neural Information Processing Systems, 2008, pp. $273-280$.

[57] D. Shahaf, C. Guestrin, Learning thin junction trees via graph cuts, in: Proceedings of the 12th International Conference on Artificial Intelligence and Statistics, Vol. 5, Proceedings of Machine Learning Research, 2009, pp. $113-120$.

[58] R. K. Ahuja, T. L. Magnanti, J. B. Orlin, Network flows: Theory, Algorithms, and Applications, Prentice Hall, 1993.

[59] D. Lowd, P. Domingos, Learning arithmetic circuits, in: Proceedings of the 24th Conference on Uncertainty in Artificial Intelligence, AUAI Press, 2008, pp. 383-392.

[60] M. Benjumeda, P. Larrañaga, C. Bielza, Learning Bayesian networks with low inference complexity, Progress in Artificial Intelligence 5 (1) (2015) $15-26$.

[61] A. Darwiche, Modeling and Reasoning with Bayesian Networks, Cambridge University Press, 2009. 
[62] D. Lowd, J. Davis, Learning Markov network structure with decision trees, in: IEEE International Conference on Data Mining, IEEE, 2010, pp. 334343.

[63] J. Van Haaren, J. Davis, Markov network structure learning: A randomized feature generation approach, in: Proceedings of the 26th AAAI Conference on Artificial Intelligence, 2012, pp. 1148-1154.

[64] J. Bekker, J. Davis, A. Choi, A. Darwiche, G. V. d. Broeck, Tractable learning for complex probability queries, in: Proceedings of the 28th International Conference on Neural Information Processing Systems, MIT Press, 2015, pp. 2242-2250.

[65] S. Holm, A simple sequentially rejective multiple test procedure, Scandinavian Journal of Statistics (1979) 65-70.

[66] J. P. Shaffer, Modified sequentially rejective multiple test procedures, Journal of the American Statistical Association 81 (395) (1986) 826-831.

[67] S. Garcia, F. Herrera, An extension on "statistical comparisons of classifiers over multiple data sets" for all pairwise comparisons, Journal of Machine Learning Research 9 (2008) 2677-2694.

[68] J. Demšar, Statistical comparisons of classifiers over multiple data sets, Journal of Machine Learning Research 7 (2006) 1-30.

[69] L. C. van der Gaag, P. R. de Waal, Multi-dimensional Bayesian network classifiers, in: Proceedings of the 3rd European Workshop on Probabilistic Graphical Models, 2006, pp. 107-114.

[70] C. Bielza, G. Li, P. Larrañaga, Multi-dimensional classification with Bayesian networks, International Journal of Approximate Reasoning 52 (6) (2011) $705-727$.

[71] M. Benjumeda, C. Bielza, P. Larrañnaga, Tractability of most probable explanations in multidimensional Bayesian network classifiers, International Journal of Approximate Reasoning 93 (Supplement C) (2018) $74-87$. 
[72] N. Friedman, The Bayesian structural EM algorithm, in: Proceedings of the 14th Conference on Uncertainty in Artificial Intelligence, Morgan Kaufmann Publishers Inc, 1998, pp. 129-138.

\section{Appendix A Proof of Theorem 1}

We use the following lemmas to prove that the compilation and optimization methods proposed in this paper always return valid ETs.

Lemma 1. Let $\mathcal{E}_{\mathcal{B}}$ be a valid $E T$ that represents $\mathcal{B}$ over $\mathcal{X}$. Given $X_{\text {out }}, X_{\text {in }} \in$ $\mathcal{X}$, the ET $\mathcal{E}_{\mathcal{B}}^{\prime}$ yielded after applying add $\left(\mathcal{E}_{\mathcal{B}}, X_{\text {out }}, X_{\text {in }}\right)$ in Algorithm 2 , is also a valid ET representing $\mathcal{B}^{\prime}$ over $\mathcal{X}$.

Proof. By cases:

We prove that, for each possible arc addition scenario, Algorithm 2 always outputs valid ETs. We show that, in each case, all the nodes are complete (i.e., for each node its cluster in $\mathcal{E}_{\mathcal{B}^{\prime}}^{\prime}$ contains its parents (Definition 9)) and sound (i.e., for each node its cluster in $\mathcal{E}_{\mathcal{B}^{\prime}}^{\prime}$ is a subset of its predecessors and itself (Definition 8)).

- Case 1: $X_{\text {out }} \in \operatorname{Pred}_{\varepsilon_{B}}\left(\phi_{X_{\text {in }}}\right)$.

This occurs when neither of the conditions in lines 4 and 6 (Algorithm 1) are fulfilled. Algorithm 1 does not produce any change in the structure of $\mathcal{E}_{\mathcal{B}}$ (see Figure 16). Hence, neither the parents nor the predecessors of each node in $\mathcal{E}_{\mathcal{B}}$ change. For each node $X_{i} \in\left(\operatorname{Pred}_{\mathcal{E}_{\mathcal{B}^{\prime}}^{\prime}}\left(\phi_{X_{\text {in }}}\right) \cap \operatorname{Desc}_{\mathcal{E}_{\mathcal{B}^{\prime}}^{\prime}}(\right.$ $\left.\left.X_{\text {out }}\right)\right) \cup\left\{\phi_{X_{\text {in }}}\right\}$, the cluster of $X_{i}$ now contains $X_{\text {out }}$, but $X_{\text {out }} \in \operatorname{Pred}_{\mathcal{E}_{B}}($ $\left.X_{i}\right)$. Hence, each $X_{i}$ is sound. As $\operatorname{Cls}_{\mathcal{E}_{\mathcal{B}^{\prime}}^{\prime}}\left(X_{i}\right) \supseteq \operatorname{Cls}_{\mathcal{E}_{\mathfrak{B}}}\left(X_{i}\right)$, each $X_{i}$ is complete, and therefore valid. There are no changes in the clusters of the other nodes. Hence, they are valid.

- Case 2: $X_{f}=\operatorname{Pa}_{\mathcal{E}_{\mathcal{B}}}\left(\phi_{X_{\text {in }}}\right) \in \operatorname{Pred}_{\mathcal{E}_{\mathcal{B}}}\left(X_{\text {out }}\right)$ (line 4 of Algorithm 2).

Here, Algorithm 1 sets $\mathrm{Pa}_{\mathcal{E}_{\mathcal{B}^{\prime}}^{\prime}}\left(\phi_{X_{\text {in }}}\right)$ to $X_{\text {out }}$ (line 5), and the predecessors and parents of the other nodes are unchanged (see Figure 17). 


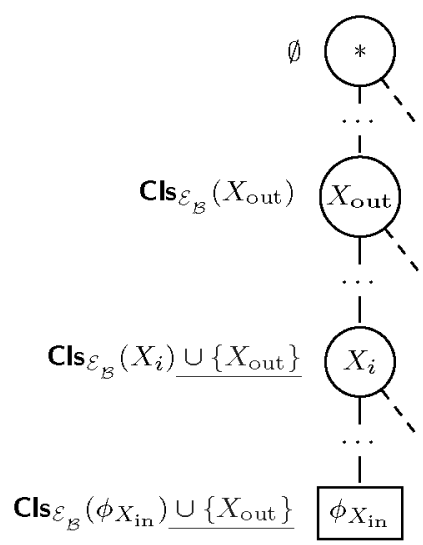

Figure 16: ET $\mathcal{E}_{\mathcal{B}^{\prime}}^{\prime}$ yielded after compiling an arc addition $X_{\text {out }} \rightarrow X_{\text {in }}$ in $\mathcal{E}_{\mathcal{B}}$ when $X_{\text {out }} \in$ $\operatorname{Pred}_{\mathcal{E}_{\mathcal{B}}}\left(\phi_{X_{\mathrm{in}}}\right)$. The value of the cluster of each node in $\mathcal{E}_{\mathcal{B}^{\prime}}^{\prime}$ is shown near to the respective node, and the changes in the clusters with respect to their value in $\mathcal{E}_{\mathcal{B}}$ are underlined

- For each node $X_{i} \in \operatorname{Pred}_{\mathcal{E}_{\mathcal{B}^{\prime}}^{\prime}}\left(\phi_{X_{\mathrm{in}}}\right) \cap \operatorname{Desc}_{\mathcal{E}_{\mathcal{B}^{\prime}}^{\prime}}\left(X_{f}\right)$, we have that $\operatorname{Cls}_{\mathcal{E}_{\mathcal{B}^{\prime}}^{\prime}}\left(X_{i}\right)=\operatorname{Cls}_{\mathcal{E}_{13}}\left(X_{i}\right) \cup \operatorname{Cls}_{\mathcal{E}_{B}}\left(\phi_{X_{\mathrm{in}}}\right)$. First, $\operatorname{Cls}_{\mathcal{E}_{\mathrm{B}}}\left(X_{i}\right) \subseteq \operatorname{Pred}_{\mathcal{E}_{\mathrm{B}}}($ $\left.X_{i}\right)=\operatorname{Pred}_{\mathcal{E}_{\mathcal{B}^{\prime}}^{\prime}}\left(X_{i}\right)$. Also, $\operatorname{Cls}_{\mathcal{E}_{\mathcal{B}}}\left(\phi_{X_{\mathrm{in}}}\right) \subseteq \operatorname{Pred}_{\mathcal{E}_{\mathcal{B}}}\left(\phi_{X_{\mathrm{in}}}\right)=\operatorname{Pred}_{\mathcal{E}_{\mathcal{B}}}($ $\left.X_{f}\right) \cup\left\{X_{f}\right\} \subseteq \operatorname{Pred}_{\mathcal{E}_{\mathcal{B}}}\left(X_{i}\right)=\operatorname{Pred}_{\mathcal{E}_{\mathcal{B}^{\prime}}^{\prime}}\left(X_{i}\right)$. Therefore, $\operatorname{Cls}_{\mathcal{E}_{\mathcal{B}^{\prime}}^{\prime}}\left(X_{i}\right) \subseteq$ $\operatorname{Pred}_{\mathcal{E}_{\mathcal{B}^{\prime}}^{\prime}}\left(X_{i}\right)$, and $X_{i}$ is sound in $\mathcal{E}_{\mathcal{B}^{\prime}}^{\prime}$. As $\operatorname{Cls}_{\mathcal{E}_{\mathcal{B}^{\prime}}^{\prime}}\left(\phi_{X_{\mathrm{in}}}\right)=\operatorname{Cls}_{\mathcal{E}_{\mathrm{B}}}\left(\phi_{X_{\mathrm{in}}}\right) \cup$ $\left\{X_{\text {out }}\right\}$ and $\operatorname{Pred}_{\mathcal{E}_{\mathcal{B}^{\prime}}^{\prime}}\left(\phi_{X_{\text {in }}}\right) \supseteq \operatorname{Pred}_{\mathcal{E}_{\mathcal{B}}}\left(\phi_{X_{\text {in }}}\right) \cup\left\{X_{\text {out }}\right\}, \phi_{X_{\text {in }}}$ is sound. The rest of the nodes are sound given that there are no changes in their clusters.

- Node $\phi_{X_{\text {in }}}$ is complete given that $X_{\text {out }}=\operatorname{Pa}_{\mathcal{E}_{\mathcal{B}^{\prime}}^{\prime}}\left(\phi_{X_{\text {in }}}\right)$ and $X_{\text {out }} \in$ $\mathrm{Cls}_{\mathcal{E}_{\mathcal{B}^{\prime}}^{\prime}}\left(\phi_{X_{\mathrm{in}}}\right)$. The rest of the nodes are complete given that $\mathrm{Cls}_{\mathcal{E}_{\mathcal{B}^{\prime}}^{\prime}}\left(X_{i}\right) \supseteq$ $\operatorname{Cls}_{\mathcal{E}_{\mathcal{B}}}\left(X_{i}\right)$ and $\operatorname{Pa}_{\mathcal{E}_{\mathcal{B}^{\prime}}^{\prime}}\left(X_{i}\right)=\operatorname{Pa} \mathcal{E}_{\mathcal{B}}\left(X_{i}\right)$ for each $X_{i} \in\left(\mathcal{X} \cup \operatorname{Leaves}\left(\mathcal{E}_{\mathcal{B}}\right)\right) \backslash$ $\left\{\phi_{X_{\text {in }}}\right\}$.

- Case 3: $X_{\text {out }} \notin \operatorname{Pred}_{\mathcal{E}_{B}}\left(\phi_{X_{\mathrm{in}}}\right)$ and $X_{f} \notin \operatorname{Pred}_{\mathcal{E}_{B}}\left(X_{\text {out }}\right)$ (line 6 of Algorithm 2).

In this case, there are two possible output ETs, $\mathcal{E}_{\mathcal{B}^{\prime}}^{1}$ and $\mathcal{E}_{\mathcal{B}^{\prime}}^{2}$ (line 8 ).

1. In $\mathcal{E}_{\mathcal{B}^{\prime}}^{1}$ (see Figure 18b), $\operatorname{Pa}_{\mathcal{E}_{\mathcal{B}^{\prime}}^{1}}\left(X_{k}\right)$ is set to $X_{\text {out }}$ (line 10): 


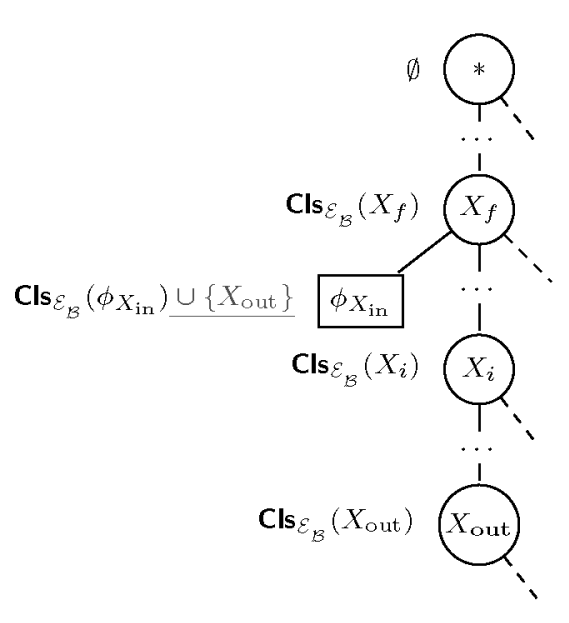

(a) $\mathcal{E}_{\mathcal{B}^{\prime}}$

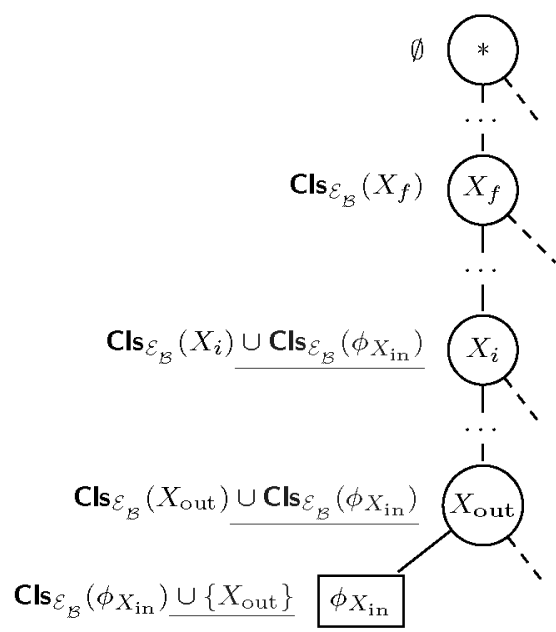

(b) $\mathcal{E}_{B^{\prime}}^{\prime}$

Figure 17: (a) $\mathcal{E}_{\mathcal{B}}$, is the ET yielded after adding $X_{\text {out }} \rightarrow X_{\text {in }}$ in $\mathcal{B}$ when $X_{f} \in \operatorname{Pred}_{\mathcal{E}_{\mathcal{B}}}\left(X_{\text {out }}\right)$ before the compilation of the arc addition; (b) $\mathcal{E}_{\mathcal{B}^{\prime}}^{\prime}$ is the result of compiling the arc addition $X_{\text {out }} \rightarrow X_{\text {in }}$ in $\mathcal{E}_{\mathcal{B}}$. The value of the cluster of each node in $\mathcal{E}_{\mathcal{B}^{\prime}}^{\prime}$ is shown near to the respective node, and the changes in the clusters with respect to their value in $\mathcal{E}_{\mathcal{B}}$ are underlined. The changes that compromise the validity of the ET are highlighted in red 
- Each node $X_{i}$ that is not in $\left(\operatorname{Pred}_{\mathcal{E}_{\xi^{\prime}}^{1}}\left(\phi_{X_{\text {in }}}\right) \cap \operatorname{Desc}_{\mathcal{E}_{\mathcal{B}^{\prime}}^{1}}\left(X_{m}\right)\right) \cup$ $\left\{\phi_{X_{\text {in }}}\right\}$ has the same parents and clusters in $\mathcal{E}_{\mathcal{B}}$ and $\mathcal{E}_{\mathcal{B}^{\prime}}^{1}$, and $\operatorname{Pred}_{\mathcal{E}_{\mathcal{B}^{\prime}}^{1}}\left(X_{i}\right) \supseteq \operatorname{Pred}_{\mathcal{E}_{\mathcal{B}}}\left(X_{i}\right)$. Hence, it is valid.

- Each node $X_{i}$ in $\operatorname{Pred}_{\mathcal{E}_{\mathcal{B}^{\prime}}}\left(X_{k}\right) \cap \operatorname{Desc}_{\mathcal{E}_{\mathcal{B}^{\prime}}^{1}}\left(X_{m}\right)$ has the same predecessors and parents in $\mathcal{E}_{\mathcal{B}}$ and $\mathcal{E}_{\mathcal{B}^{\prime}}^{1}$, and $\operatorname{Cls}_{\mathcal{E}_{\mathcal{B}^{\prime}}}\left(X_{i}\right)=\operatorname{Cls}_{\mathcal{E}_{\mathcal{B}}}\left(X_{i}\right) \cup$ $\operatorname{Cls}_{\mathcal{E}_{\mathfrak{B}}}\left(X_{k}\right) \backslash\left\{X_{k}\right\}$ (making $X_{i}$ complete). As Cls S $_{\mathcal{E}_{\mathfrak{B}^{\prime}}^{1}}\left(X_{k}\right) \backslash\left\{X_{k}\right\} \subseteq$ $\operatorname{Pred}_{\mathcal{E}_{B}}\left(X_{k}\right) \subseteq \operatorname{Pred}_{\mathcal{E}_{\mathcal{B}^{\prime}}^{1}}\left(X_{i}\right)$, each $X_{i}$ is also sound. Thus, each $X_{i}$ is valid.

- For each $X_{i} \in\left(\operatorname{Pred}_{\mathcal{E}_{\mathcal{B}^{\prime}}^{1}}\left(\phi_{X_{\mathrm{in}}}\right) \cup\left\{\phi_{X_{\mathrm{in}}}\right\}\right) \cap \operatorname{Desc}_{\mathcal{E}_{\mathcal{B}^{\prime}}^{1}}\left(X_{\mathrm{out}}\right), \operatorname{Cls}_{\mathcal{E}_{\mathcal{B}^{\prime}}^{1}}\left(X_{i}\right)=$ $\operatorname{Cls}_{\mathcal{E}_{\mathcal{B}}}\left(X_{i}\right) \cup\left\{X_{\text {out }}\right\}, \operatorname{Pred}_{\mathcal{E}_{\mathcal{B}^{\prime}}}\left(X_{i}\right) \cup\left\{X_{i}\right\} \supseteq \operatorname{Cls}_{\mathcal{E}_{\mathcal{B}}}\left(X_{i}\right) \cup\left\{X_{\text {out }}\right\}$, $\mathrm{Pa}_{\mathcal{E}_{\mathcal{B}^{\prime}}^{1}}\left(X_{i}\right)=\mathrm{Pa}_{\mathcal{E}_{\mathfrak{B}}}\left(X_{i}\right)$ if $X_{i} \neq X_{k}$, and $\mathrm{Pa}_{\mathcal{E}_{\mathcal{B}^{\prime}}^{1}}\left(X_{k}\right)=X_{\text {out }}$. Hence, $X_{i}$ is valid.

As each node in $\mathcal{E}_{\mathcal{B}^{\prime}}^{1}$ is valid, $\mathcal{E}_{\mathcal{B}^{\prime}}^{1}$ is valid.

2. In $\mathcal{E}_{\mathcal{B}^{\prime}}^{2}$ (see Figure $18 \mathrm{c}$ ), $\mathrm{Pa}_{\mathcal{E}_{\mathcal{B}^{\prime}}}\left(X_{h}\right)$ is set to $X_{f}$ (line 12).

- Each node has the same parent in $\mathcal{E}_{\mathcal{B}^{\prime}}^{2}$ and $\mathcal{E}_{\mathcal{B}}$, with the exception of $\phi_{X_{\mathrm{in}}}$ and $X_{h}$, where $\mathrm{Pa}_{\mathcal{E}_{\mathcal{E}^{\prime}}^{2}}\left(\phi_{X_{\mathrm{in}}}\right)=X_{\text {out }}$ (line 13) and $\mathrm{Pa}_{\mathcal{E}_{\mathcal{B}^{\prime}}^{2}}\left(X_{h}\right)=X_{f}$. All the nodes are complete, given that $X_{\text {out }} \in \operatorname{Cls}_{\mathcal{E}_{\mathcal{B}^{\prime}}^{2}}\left(\phi_{X_{\text {in }}}\right), \operatorname{Cls}_{\mathcal{E}_{\mathcal{B}^{\prime}}^{2}}\left(X_{h}\right) \supseteq \operatorname{Cls}_{\mathcal{E}_{\mathcal{B}_{3}}}\left(\phi_{X_{\mathrm{in}}}\right) \supseteq\left\{X_{f}\right\}$, and for each other node $X_{i}, \operatorname{Cls}_{\mathcal{E}_{\mathcal{E}^{\prime}}^{2}}\left(X_{i}\right) \supseteq \operatorname{Cls}_{\mathcal{E}_{\mathcal{B}}}\left(X_{i}\right)$.

- For each node $X_{i}$ not in $\left(\operatorname{Pred}_{\mathcal{E}_{\mathcal{B}^{\prime}}^{2}}\left(\phi_{X_{\mathrm{in}}}\right) \cap \operatorname{Desc}_{\mathcal{E}_{\mathcal{B}^{\prime}}^{2}}\left(X_{m}\right)\right) \cup\left\{\phi_{X_{\mathrm{in}}}\right\}$, the clusters of $X_{i}$ are the same in $\mathcal{E}_{\mathcal{B}^{\prime}}^{2}$ and in $\mathcal{E}_{\mathcal{B}}$ and $\operatorname{Pred}_{\mathcal{E}_{\mathcal{B}^{\prime}}^{2}}($ $\left.X_{i}\right) \supseteq \operatorname{Pred}_{\mathcal{E}_{\mathcal{B}}}\left(X_{i}\right)$. Hence each $X_{i}$ is sound.

- For each $X_{i} \in \operatorname{Pred}_{\mathcal{E}_{B^{\prime}}^{2}}\left(X_{h}\right) \cap \operatorname{Desc}_{\mathcal{E}_{B^{\prime}}^{2}}\left(X_{m}\right), \operatorname{Cls}_{\mathcal{E}_{\mathcal{B}^{\prime}}^{2}}\left(X_{i}\right)=\operatorname{Cls}_{\mathcal{E}_{B_{B}}}\left(X_{i}\right) \cup$ $\operatorname{Cls}_{\mathcal{E}_{\mathcal{B}}}\left(X_{h}\right) \backslash\left\{X_{h}\right\}$ and $\operatorname{Cls}_{\mathcal{E}_{\mathcal{B}}}\left(X_{h}\right) \backslash\left\{X_{h}\right\} \subseteq \operatorname{Pred}_{\mathcal{E}_{\mathcal{B}}}\left(X_{h}\right) \subseteq$ $\operatorname{Pred}_{\mathcal{E}_{\mathcal{B}}}\left(X_{i}\right)=\operatorname{Pred}_{\mathcal{E}_{\mathcal{B}^{\prime}}^{2}}\left(X_{i}\right)$. Thus, each $X_{i}$ is sound.

- For each $X_{i} \in \operatorname{Pred}_{\mathcal{E}_{\mathcal{B}^{\prime}}^{2}}\left(\phi_{X_{\text {in }}}\right) \cap \operatorname{Desc}_{\mathcal{E}_{\mathcal{B}^{\prime}}^{2}}\left(X_{f}\right), \operatorname{Cls}_{\mathcal{E}_{\mathcal{E}^{\prime}}^{2}}\left(X_{i}\right)=$ $\operatorname{Cls}_{\mathcal{E}_{\mathcal{B}}}\left(X_{i}\right) \cup \operatorname{Cls}_{\mathcal{E}_{\mathcal{B}}}\left(\phi_{X_{\mathrm{in}}}\right)$ and $\operatorname{Pred}_{\mathcal{E}_{\mathcal{B}^{\prime}}^{2}}\left(X_{i}\right)=\operatorname{Pred}_{\mathcal{E}_{\mathcal{B}}}\left(X_{i}\right) \cup$ $\operatorname{Pred}_{\mathcal{E}_{\mathcal{B}}}\left(\phi_{X_{\text {in }}}\right) \supseteq \operatorname{Cls}_{\mathcal{E}_{\mathcal{B}}}\left(X_{i}\right) \backslash\left\{X_{i}\right\} \cup \operatorname{Cls}_{\mathcal{E}_{\mathcal{B}}}\left(\phi_{X_{\text {in }}}\right)$. Hence, $X_{i}$ is sound. 
- Nøde $\phi_{X_{\text {in }}}$ cøntains $X_{\bullet \text { ut }}$ in its cluster and predecessørs in $\mathcal{E}_{\mathcal{B}^{\prime}}^{2}$. Hence, $\operatorname{Cls}_{\mathcal{E}_{\mathcal{B}^{\prime}}^{2}}\left(\phi_{X_{\text {in }}}\right)=\operatorname{Cls}_{\mathcal{E}_{\mathcal{B}}}\left(\phi_{X_{\text {in }}}\right) \cup\{X \bullet$ ut $\} \subseteq \operatorname{Pred}_{\mathcal{E}_{\mathcal{B}}}\left(\phi_{X_{\text {in }}}\right) \cup$ $\left\{X_{\bullet \text { ut }}\right\} \subseteq \operatorname{Pred}_{\mathcal{E}_{\mathcal{B}^{\prime}}^{2}}\left(\phi_{X_{\text {in }}}\right)$, making $\phi_{X_{\text {in }}}$ søund.

As every nøde in $\mathcal{E}_{\mathcal{B}^{\prime}}^{2}$, is søund and complete, $\mathcal{E}_{\mathcal{B}^{\prime}}^{2}$ is valid.

Lemma 2. Let $\mathcal{E}_{\mathcal{B}}$ be $\boldsymbol{a}$ valid ET that represents $\mathcal{B} \bullet$ ver $\mathcal{X}$. Given $X \bullet$ ,,$X_{\text {in }} \in$ $\mathcal{X}$, the ET $\mathcal{E}_{\mathcal{B}^{\prime}}^{\prime}$ that represents $\mathcal{B}^{\prime} \bullet$ utput after applying remøve $\left(\mathcal{E}_{\mathcal{B}}, X_{\bullet}, X_{i n}\right)$ in Algorithm 3, is als• valid.

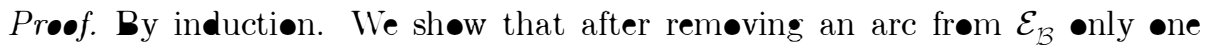

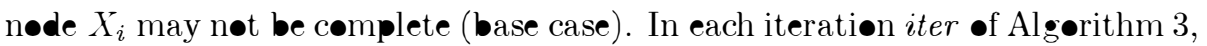

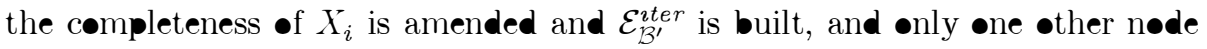
$X_{i}^{\prime}$, which was a predecessør of $X_{i}$ in the previøus ET, may nøt be complete after the change. It is evident that eventually node $X_{i}^{\prime}$ will be complete (e.g., when the parent $\bullet X_{i}^{\prime}$ is the røot node $*$ ).

Base case:

Given a valid ET $\mathcal{E}_{\mathcal{B}}$, remøving arc $X_{\bullet \text { ut }} \rightarrow X_{\text {in }}$ frøm $\mathcal{B}$ will prøduce an ET $\mathcal{E}_{\mathcal{B}^{\prime}}$. For each $X_{h} \in \mathcal{X} \cup \operatorname{Leaves}\left(\mathcal{E}_{\mathcal{B}^{\prime}}\right), \operatorname{Pred}_{\mathcal{E}_{\mathcal{B}^{\prime}}}\left(X_{h}\right)=\operatorname{Pred}_{\mathcal{E}_{\mathcal{B}}}\left(X_{h}\right)$, $\mathbf{P a}_{\mathcal{E}_{\mathcal{B}^{\prime}}}\left(X_{h}\right)=\mathbf{P a}_{\mathcal{E}_{\mathcal{B}}}\left(X_{h}\right), \operatorname{Cls}_{\mathcal{E}_{\mathcal{B}}}\left(X_{h}\right) \supseteq \operatorname{Cls}_{\mathcal{E}_{\mathcal{B}^{\prime}}}\left(X_{h}\right) \supseteq \operatorname{Cls}_{\mathcal{E}_{\mathcal{B}}}\left(X_{h}\right) \backslash\left\{X_{\bullet \text { ut }}\right\}$ if $X_{h} \in \operatorname{Desc}_{\mathcal{E}_{\mathcal{B}^{\prime}}}\left(X_{\bullet \mathrm{ut}}\right) \cap \operatorname{Pred}_{\mathcal{E}_{\mathcal{B}^{\prime}}}\left(\phi_{X_{\text {in }}}\right) \cup\left\{\phi_{X_{\text {in }}}\right\}$, and $\operatorname{Cls}_{\mathcal{E}_{\mathcal{B}^{\prime}}}\left(X_{h}\right)=\operatorname{Cls}_{\mathcal{E}_{\mathcal{B}}}\left(X_{h}\right)$

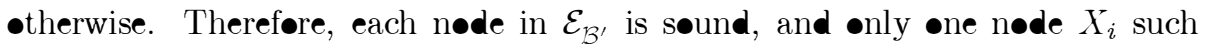
that $X_{i} \in \mathbf{C h}_{\mathcal{E}_{\mathcal{B}^{\prime}}}\left(X_{\bullet \text { ut }}\right) \cap\left(\boldsymbol{P r e d}_{\mathcal{E}_{\mathcal{B}^{\prime}}}\left(\boldsymbol{\phi}_{X_{\text {in }}}\right) \cup\left\{\boldsymbol{\phi}_{X_{\text {in }}}\right\}\right)$ may nøt be complete.

Iterative step:

Assume that $\mathcal{E}_{\mathcal{B}^{\prime}}^{1}$ (Figure 19a) is søund and only nøde $X_{i}$ is nøt complete. Algørithm 3 sets $\mathbf{P a}_{\mathcal{E}_{\mathcal{B}^{\prime}}^{1}}\left(X_{i}\right)=X_{j}$ (line 7), that is, the deepest nøde in $\mathcal{E}_{\mathcal{B}^{\prime}}^{1}$ belønging t• $\operatorname{Cls}_{\mathcal{E}_{\mathcal{B}}}\left(X_{i}\right) \backslash\left\{X_{i}\right\}$ (line 6). Hence, all $X_{i}$ and their descendants are søund. Thus, nøde $X_{i}$ is complete in the resulting ET $\mathcal{E}_{\mathcal{B}^{\prime}}^{2}$ (Figure 19b). Node

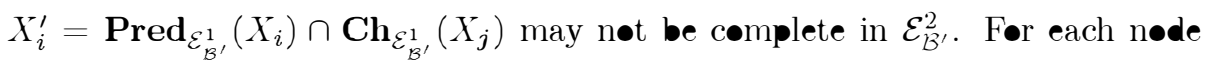
$X_{h} \in \operatorname{Pred}_{\mathcal{E}_{\mathcal{B}^{\prime}}^{1}}\left(X_{i}\right) \cap \operatorname{Desc}_{\mathcal{E}_{\mathcal{B}^{\prime}}^{1}}\left(X_{i}^{\prime}\right), \operatorname{Cls}_{\mathcal{E}_{\mathcal{B}^{\prime}}^{2}}\left(X_{h}\right) \subseteq \operatorname{Cls}_{\mathcal{E}_{\mathcal{B}^{\prime}}^{1}}\left(X_{h}\right) \backslash \operatorname{Cls}_{\mathcal{E}_{\mathcal{B}^{\prime}}^{1}}\left(X_{i}\right)$ and 


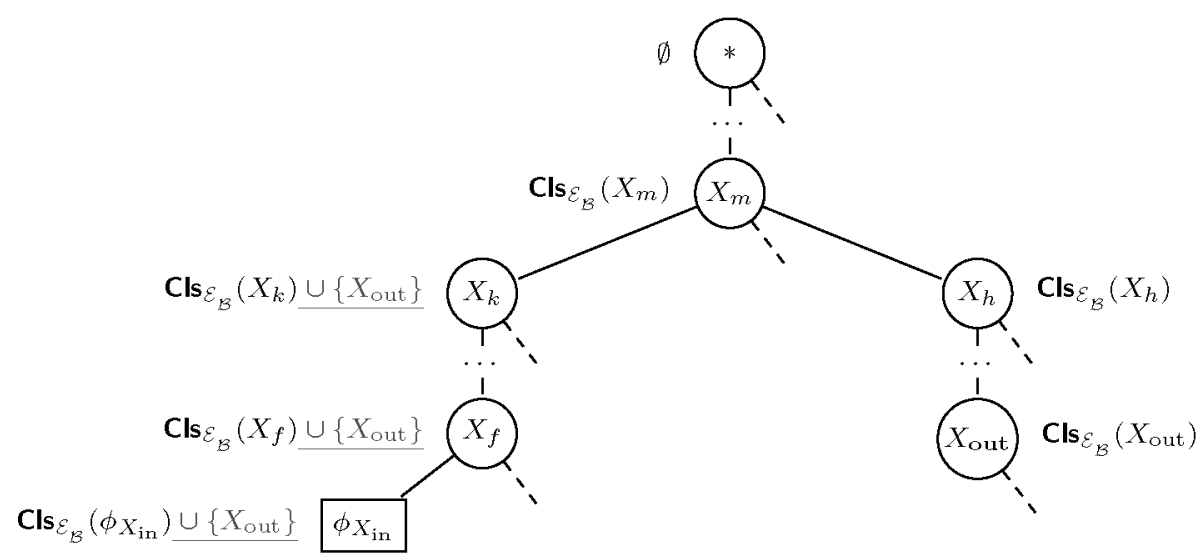

(a) $\mathcal{E}_{\mathcal{B}^{\prime}}$

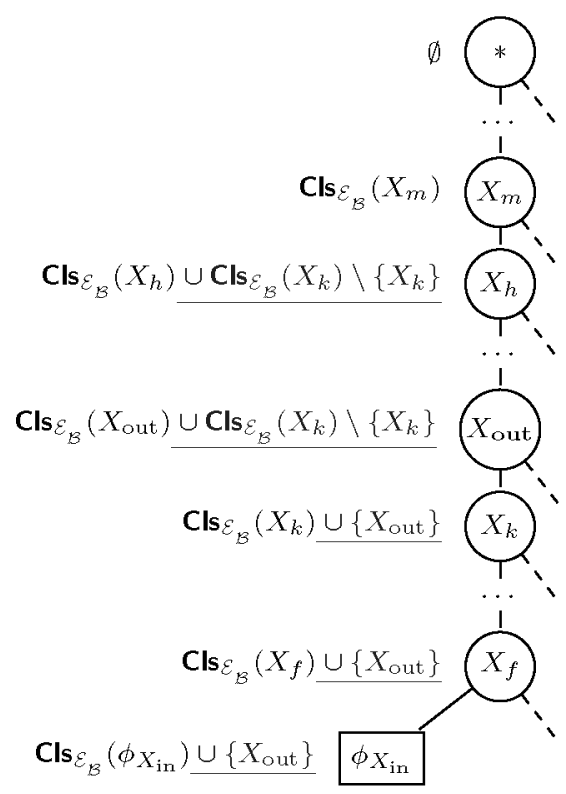

(b) $\mathcal{E}_{\mathcal{B}^{\prime}}^{1}$

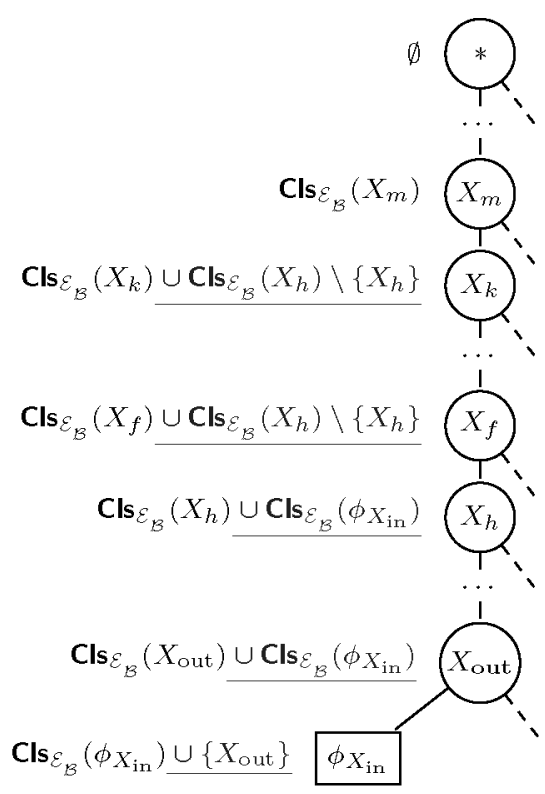

(c) $\mathcal{E}_{\mathcal{B}^{\prime}}^{2}$

Figure 18: $\mathcal{E}_{\mathcal{B}^{\prime}}$ (Figure $18 \mathrm{a}$ ) is the ET yielded after adding $X_{\text {out }} \rightarrow X_{\text {in }}$ in $\mathcal{B}$ when $X_{\text {out }} \notin$ $\operatorname{Pred}_{\mathcal{E}_{\mathcal{B}}}\left(\phi_{X_{\text {in }}}\right)$ and $X_{f} \notin \operatorname{Pred}_{\mathcal{E}_{\mathcal{B}}}\left(X_{\text {out }}\right)$ before the compilation of the arc addition. $\mathcal{E}_{\mathcal{B}^{\prime}}^{1}$ (Figure 18b) and $\mathcal{E}_{\mathcal{B}^{\prime}}^{2}$ (Figure 18c) correspond to the two possible outcomes of compiling the arc addition. The value of the cluster of each node in $\mathcal{E}_{\mathcal{B}^{\prime}}^{\prime}$, is shown near to the respective node, and the changes in the clusters with respect to their value in $\mathcal{E}_{\mathcal{B}}$ are underlined. The changes that compromise the validity of the ET are highlighted in red 

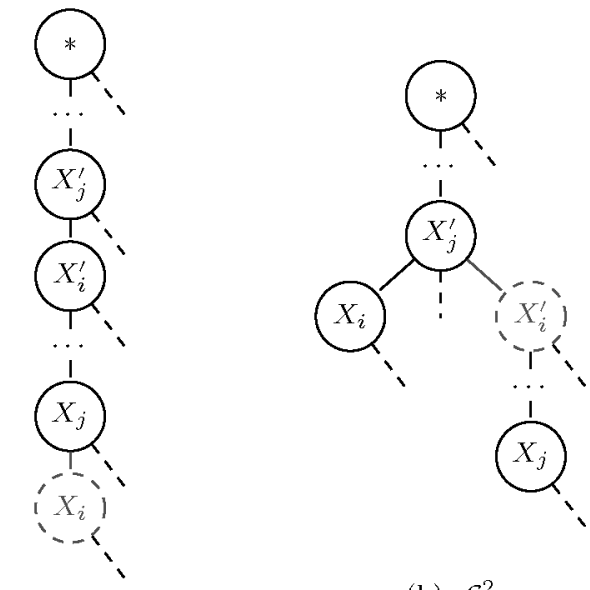

(b) $\mathcal{E}_{\mathcal{B}^{\prime}}^{2}$

(a) $\mathcal{E}_{\mathcal{B}^{\prime}}^{1}$

Figure 19: (a) $\mathcal{E}_{\mathcal{B}^{\prime}}^{1}$ is the ET visited at an iterative step of compiling an arc removal; (b) $\mathcal{E}_{\mathcal{B}^{\prime}}^{2}$ is the result of performing this iterative step

$\operatorname{Pa}_{\mathcal{E}_{\mathcal{B}^{\prime}}^{\prime}}\left(X_{h}\right) \notin \operatorname{Cls}_{\mathcal{E}_{\mathcal{B}^{\prime}},}\left(X_{i}\right)$. Hence, each $X_{h}$ is complete in $\mathcal{E}_{\mathcal{B}^{\prime}}^{2}$. The other nodes have the same clusters in $\mathcal{E}_{\mathcal{B}^{\prime}}^{1}$, and in $\mathcal{E}_{\mathcal{B}^{\prime}}^{2}$. Hence, they are complete.

Lemma 3. Let $\mathcal{E}_{\mathcal{B}}$ be a valid ET that represents $\mathcal{B}$ over $\mathcal{X}$. Given $X_{i} \in \mathcal{X}$, the $E T \mathcal{E}_{\mathcal{B}}^{\prime}$ representing $\mathcal{B}^{\prime}$ yielded after applying $\operatorname{swap}\left(\mathcal{E}_{\mathcal{B}}, X_{i}\right)$ in Algorithm 4, is also valid.

Proof. Let $X_{p}$ be the parent of $X_{i}$ in $\mathcal{E}_{\mathcal{B}}$ (Figure 20a). Next, we prove that each node in $\mathcal{E}_{\mathcal{B}}^{\prime}$ (Figure $20 \mathrm{~b}$ ) is valid after the swap:

- Each node $X_{j}$ in $\left(\operatorname{Desc}_{\mathcal{E}_{\mathcal{B}}}\left(X_{p}\right) \backslash\left(\operatorname{Desc}_{\mathcal{E}_{\mathcal{B}}}\left(X_{i}\right) \cup\left\{X_{i}\right\}\right)\right) \cup \operatorname{Pred}_{\mathcal{E}_{\mathcal{B}}}\left(X_{p}\right)$ has the same parent and clusters in $\mathcal{E}_{\mathcal{B}}^{\prime}$, and $\operatorname{Pred}_{\mathcal{E}_{B}^{\prime}}\left(X_{j}\right) \supset \operatorname{Pred}_{\mathcal{E}_{\mathcal{B}}}\left(X_{j}\right)$. Hence, each $X_{j}$ is valid in $\mathcal{E}_{\mathcal{B}}^{\prime}$.

- Let us divide the descendants of $X_{i}$ in $\mathcal{E}_{\mathcal{B}}$ into two subsets $D_{1}$ and $D_{2}$. Let $C_{1}$ be the children of $X_{i}$ in $\mathcal{E}_{\mathcal{B}}$ that do not contain $X_{p}$ in its cluster. We use $D_{1}$ to refer to the nodes in $\operatorname{Desc}_{\mathcal{E}_{B}}\left(X_{i}\right)$ such that for each $X_{j} \in D_{1}$, $\left(\operatorname{Pred}_{\mathcal{E}_{\mathcal{B}}}\left(X_{j}\right) \cup\left\{X_{j}\right\}\right) \cap C_{1} \neq \emptyset$, and $D_{2}$ to refer to the nodes in $\operatorname{Desc}_{\mathcal{E}_{\mathcal{B}}}($ $\left.X_{i}\right) \backslash D_{1}$ 


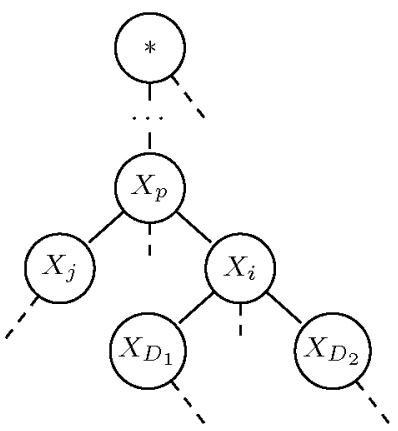

(a) $\mathcal{E}_{B}$

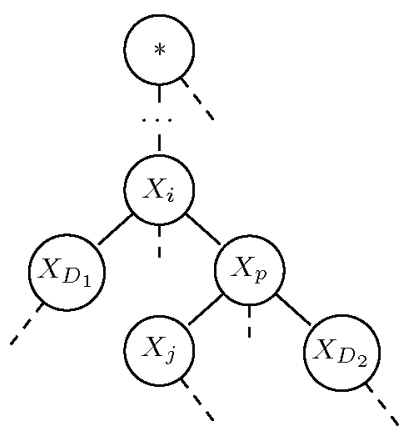

(b) $\mathcal{E}_{\mathcal{B}}^{\prime}$

Figure 20: Swap of $X_{i}$ and $X_{p} \cdot \mathcal{E}_{\mathcal{B}}$ (a) and $\mathcal{E}_{\mathcal{B}}^{\prime}$ (b) correspond to the ETs before and after swapping $X_{i}$ and $X_{p}$, respectively. Note that if $\mathcal{E}_{\mathcal{B}}$ is valid, $\mathcal{E}_{\mathcal{B}}^{\prime}$ is also valid

Each node $X_{j}$ in $D_{1}$ has the same parent and cluster in $\mathcal{E}_{\mathcal{B}}$ and $\mathcal{E}_{\mathcal{B}}^{\prime}$, and $\operatorname{Pred}_{\mathcal{E}_{B}}\left(X_{j}\right)=\operatorname{Pred}_{\mathcal{E}_{\mathcal{B}}^{\prime}}\left(X_{j}\right) \backslash\left\{X_{p}\right\}$. As $X_{p} \notin \operatorname{Cls}_{\mathcal{E}_{B}}\left(X_{j}\right)$, the respective $X_{j}$ are valid in $\mathcal{E}_{B}^{\prime}$.

Each $X_{j}$ in $D_{2}$ has the same predecessors and clusters in $\mathcal{E}_{\mathcal{B}}$ and $\mathcal{E}_{\mathcal{B}}^{\prime}$. If $X_{j} \notin \mathbf{C h}_{\mathcal{E}_{\mathcal{B}}}\left(X_{i}\right), X_{j}$ has the same parent in $\mathcal{E}_{\mathcal{B}}$ and $\mathcal{E}_{\mathcal{B}}^{\prime}$. Otherwise, $\operatorname{Pa}_{\mathcal{E}_{\mathcal{B}}^{\prime}}($ $\left.X_{j}\right)=X_{p}$ and $X_{p} \in \operatorname{Cls}_{\mathcal{E}_{\mathcal{B}}}\left(X_{j}\right)$. Thus, each $X_{j}$ is valid in $\mathcal{E}_{\mathcal{B}}^{\prime}$.

- $X_{i} \in \mathbf{C l s}_{\mathcal{E}_{\mathcal{B}}^{\prime}}\left(X_{p}\right)$, given that there are nodes in $D_{2}$ whose cluster contains $X_{i}$ (otherwise $\mathcal{E}_{\mathcal{B}}$ would not be complete). This means that $X_{p}$ is complete for $\mathcal{E}_{\mathcal{B}}^{\prime}$. As $\operatorname{Cls}_{\mathcal{E}_{\mathcal{B}}^{\prime}}\left(X_{p}\right) \subseteq \operatorname{Cls}_{\mathcal{E}_{\mathcal{B}}}\left(X_{p}\right) \cup\left\{X_{i}\right\}$ and $\operatorname{Pred}_{\mathcal{E}_{\mathcal{B}}^{\prime}}\left(X_{p}\right)=\operatorname{Pred}_{\mathcal{E}_{\mathcal{B}}}($ $\left.X_{p}\right) \cup\left\{X_{i}\right\}, X_{p}$ is sound, and therefore valid for $\mathcal{E}_{\mathcal{B}}^{\prime}$.

- As Cls $\operatorname{Cls}_{\mathcal{E}_{B}^{\prime}}\left(X_{i}\right)=\left(\operatorname{Cls}_{\mathcal{E}_{\mathcal{B}}}\left(X_{i}\right) \cup \operatorname{Cls}_{\mathcal{E}_{\mathcal{B}}}\left(X_{p}\right)\right) \backslash\left\{X_{p}\right\}, \operatorname{Pred}_{\mathcal{E}_{B}^{\prime}}\left(X_{i}\right)=\operatorname{Pred}_{\mathcal{E}_{\mathcal{B}}}($ $\left.\left.X_{i}\right) \cup \operatorname{Pred}_{\mathcal{E}_{\mathcal{B}}}\left(X_{p}\right)\right) \backslash\left\{X_{p}\right\}$, and the parent of $X_{i}$ in $\mathcal{E}_{\mathcal{B}}^{\prime}$ is the parent of $X_{p}$ in $\mathcal{E}_{\mathcal{B}}, X_{i}$ is valid for $\mathcal{E}_{\mathcal{B}}^{\prime}$.

We have shown that each node in $\mathcal{X} \cup \operatorname{Leaves}\left(\mathcal{E}_{\mathcal{B}}^{\prime}\right)$ is valid in $\mathcal{E}_{\mathcal{B}}^{\prime}$. Thus, $\mathcal{E}_{\mathcal{B}}^{\prime}$ is valid.

Finally, Theorem 1 can be proved using the above lemmas. 
Theorem 1. Let $\mathcal{E}_{\mathcal{B}}$ be a valid ET $\bullet$ ver $\mathcal{X}$, and $\mathcal{E}_{\mathcal{B}^{\prime}}^{\prime}$ the result of incrementally compiling $\bullet \mathcal{E}_{\mathcal{B}}$ any local change in $\mathcal{B}$ using Algorithms 2 and 3 and optimizing the resulting ET using Algorithm 5. Then $\mathcal{E}_{\mathcal{B}^{\prime}}^{\prime}$ is a valid ET.

Prøof. By Lemmas 1 and 2 we kn॰w that if $\mathcal{E}_{\mathcal{B}}$ is valid, the tree returned after

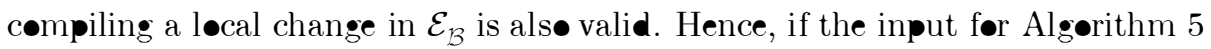
is a valid ET, we kn॰w, by Lemma 3 (the optimization is composed of a sequence -f swaps), that it will alsø return a valid ET.

\section{Appendix B Prøof of Theorem 2}

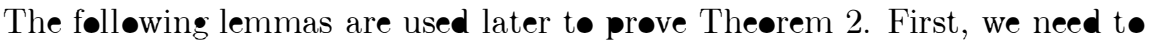
kn॰w the computational cost $\bullet$ outputting the cluster of a nøde in an ET.

Lemma 4. Let $\mathcal{E}_{\mathcal{B}}$ be an ET øver $\mathcal{X}=\left\{X_{1}, \ldots, X_{n}\right\}$. The cluster of a node $X_{i} \in \mathcal{X}$ can be computed in time $\boldsymbol{O}\left(\left|\boldsymbol{C h}_{\mathcal{E}_{\mathcal{B}}}\left(X_{i}\right)\right| \cdot\right.$ width $\left.\left(\mathcal{E}_{\mathcal{B}}\right)\right)$ given the clusters of the nødes in $\boldsymbol{C h}_{\mathcal{E}_{\mathcal{B}}}\left(X_{i}\right)$.

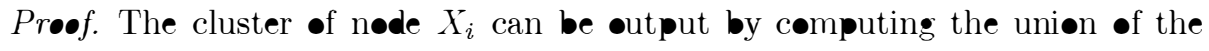

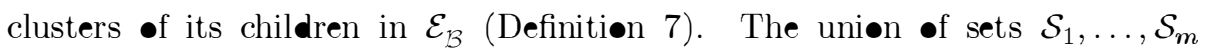
can be computed in time $\left|\mathcal{S}_{1}\right|+\cdots+\left|\mathcal{S}_{m}\right|$. As the size $\bullet$ each cluster in $\mathcal{E}_{\mathcal{B}}$ is less than $\bullet$ equal to $\operatorname{width}\left(\mathcal{E}_{\mathcal{B}}\right)+1$, then $\sum_{X_{j} \in \mathbf{C h}_{\mathcal{E}}\left(X_{i}\right)}\left|\mathbf{C l s}_{\mathcal{E}_{\mathcal{B}}}\left(X_{j}\right)\right| \leq$

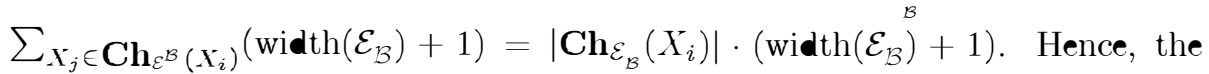
cluster $\bullet X_{i}$ can be output in time $\boldsymbol{O}\left(\left|\mathbf{C h}_{\mathcal{E}_{\mathcal{B}}}\left(X_{i}\right)\right| \cdot \operatorname{width}\left(\mathcal{E}_{\mathcal{B}}\right)\right)$.

Lemma 5. Let $\mathcal{E}_{\mathcal{B}}$ be an ET •ver $\mathcal{X}=\left\{X_{1}, \ldots, X_{n}\right\}$. All the clusters in $\mathcal{E}_{\mathcal{B}}$ can be computed in time $\boldsymbol{O}\left(n \cdot\right.$ width $\left.\left(\mathcal{E}_{\mathcal{B}}\right)\right)$.

Prøf. The cluster $\bullet$ a leaf nøde is its dømain. The cluster $\bullet$ the inner nødes

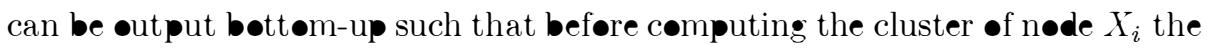

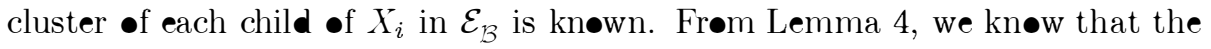
cluster of each $X_{i} \in \mathcal{X}$ can be out put in time $\boldsymbol{\bullet}\left(\left|\mathbf{C h}_{\mathcal{E}_{\mathcal{B}}}\left(X_{i}\right)\right| \cdot \operatorname{width}\left(\mathcal{E}_{\mathcal{B}}\right)\right)$. Hence, the clusters $\bullet$ all the n๑des in $\mathcal{X}$ can be computed in time $\boldsymbol{O}\left(\sum_{X_{i} \in \mathcal{X}} \mid \mathbf{C h}_{\mathcal{E}_{\mathcal{B}}}(\right.$ $\left.\left.X_{i}\right) \mid \cdot \operatorname{width}\left(\mathcal{E}_{\mathcal{B}}\right)\right)$. As $\sum_{X_{i} \in \mathcal{X}}\left|\mathbf{C h}_{\mathcal{E}_{\mathcal{B}}}\left(X_{i}\right)\right| \cdot \operatorname{width}\left(\mathcal{E}_{\mathcal{B}}\right)<2 n \cdot \operatorname{width}\left(\mathcal{E}_{\mathcal{B}}\right)$ (there are 


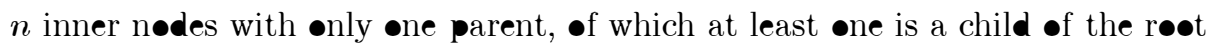
node $*$, and $n$ edges including inner and leaf nodes), all the clusters $\bullet \mathcal{E}_{\mathcal{B}}$ can be computed in time $\boldsymbol{O}\left(\boldsymbol{n} \cdot \operatorname{width}\left(\mathcal{E}_{\mathcal{B}}\right)\right)$.

A løcal change in an ET $\mathcal{E}_{\mathcal{B}}$ produces changes in the clusters of the tree,

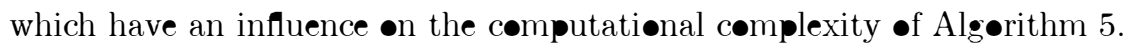

Lemma 6. Let $\mathcal{E}_{\mathcal{B}}$ be a valid ET, and $\mathcal{E}_{\mathcal{B}}^{\prime}$ the result of swapping (Algorithm 4) a n॰de $X_{i}$ and its parent in $\mathcal{E}_{\mathcal{B}}$. Then

$$
\text { width }\left(\mathcal{E}_{B}^{\prime}\right) \leq 2 \cdot \text { width }\left(\mathcal{E}_{B}\right)
$$

Prøof. After swapping $X_{i}$ and its parent $X_{p}$ in $\mathcal{E}_{\mathcal{B}}$, ønly the clusters of $X_{i}$ and $X_{p}$ may change. On the one hand, $\operatorname{Cls}_{\mathcal{E}_{\mathcal{B}}^{\prime}}\left(X_{p}\right) \subseteq \operatorname{Cls}_{\mathcal{E}_{\mathcal{B}}}\left(X_{p}\right)$. Hence, the width

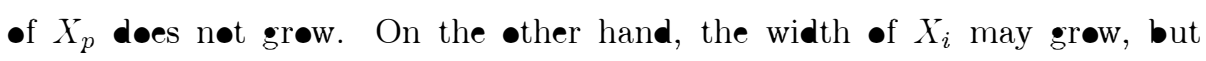
$\operatorname{Cls}_{\mathcal{E}_{\mathcal{B}}^{\prime}}\left(X_{i}\right) \subseteq\left(\operatorname{Cls}_{\mathcal{E}_{\mathcal{B}}}\left(X_{i}\right) \cup \operatorname{Cls}_{\mathcal{E}_{\mathcal{B}}}\left(X_{p}\right)\right) \backslash\left\{X_{p}\right\}$. Hence, the width $\bullet \operatorname{Cls}_{\mathcal{E}_{\mathcal{B}}^{\prime}}\left(X_{i}\right)$ is less than $\left|\operatorname{Cls}_{\mathcal{E}_{\mathcal{B}}}\left(X_{i}\right)\right|+\left|\operatorname{Cls}_{\mathcal{E}_{\mathcal{B}}}\left(X_{p}\right)\right|-1 \leq 2\left(\right.$ width $\left.\left(\mathcal{E}_{\mathcal{B}}\right)\right)+1$. This means that $\operatorname{width}\left(\mathcal{E}_{\mathcal{B}}^{\prime}\right) \leq 2 \cdot \operatorname{width}\left(\mathcal{E}_{\mathcal{B}}\right)$.

Next, we bound the time complexity of the compilation and optimization methods.

Lemma 7. Let $\mathcal{E}_{\mathcal{B}}$ be a valid ET øver $\mathcal{X}=\left\{X_{1}, \ldots, X_{n}\right\}$. The addition $\bullet$ any are in $\mathcal{B}$ can be compiled in $\mathcal{E}_{\mathcal{B}}$ in time $\boldsymbol{O}\left(n^{2}\right)$ by Algorithm 2.

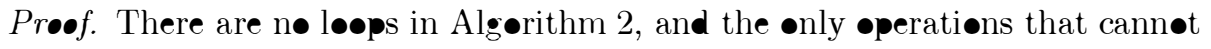
be completed in time $\boldsymbol{O}(1)$ are:

- The intersection performed to compute $X_{m}, X_{k}$ and $X_{h}$ (lines 7, 9 and 11 •f Algørithm 2), which can be computed in $\boldsymbol{O}(\boldsymbol{n})$.

- The widht of $\mathcal{E}_{\mathcal{B}^{\prime}}^{1}$ and $\mathcal{E}_{\mathcal{B}^{\prime}}^{2}$. We need t॰ output first the clusters $\bullet \mathcal{E}_{\mathcal{B}^{\prime}}^{1}$ and $\mathcal{E}_{\mathcal{B}^{\prime}}^{2}$, which can be •btained in time $\boldsymbol{O}\left(\boldsymbol{n} \cdot\right.$ width $\left.\left(\mathcal{E}_{\mathcal{B}}\right)\right)$. The width $\bullet \mathcal{E}_{\mathcal{B}}$ is the length $\bullet$ its largest cluster munus one (Definition 11), which takes $\boldsymbol{O}(\boldsymbol{n})$. The complete precess takes $\boldsymbol{O}\left(n \cdot\right.$ width $\left.\left(\mathcal{E}_{\mathcal{B}}\right)+n\right)=\boldsymbol{O}\left(n \cdot \operatorname{width}\left(\mathcal{E}_{\mathcal{B}}\right)\right) \leq$ $\left(n^{2}\right)$. 
Therefore, the addition $\bullet$ an arc can be compiled in time $\boldsymbol{O}\left(n^{2}\right)$.

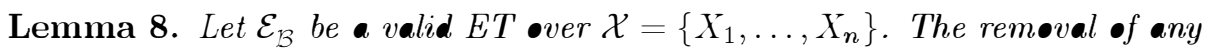
arc in $\mathcal{B}$ can be compiled in $\mathcal{E}_{\mathcal{B}}$ in time $\boldsymbol{\Theta}\left(n^{2} \cdot\right.$ width $\left.\left(\mathcal{E}_{\mathcal{B}}\right)\right)$ by Algorithm 3.

Prøof. In each iteratiøn, Algørithm 3 checks if a nøde $X_{i}$ contains its current parent in its cluster (line 5); else, the new parent $\bullet X_{i}$ is set t• the deepest nøde $X_{j}^{\prime}$, which appears in the cluster $\bullet X_{i}$ (line 6). Then, the child $\bullet X_{j}^{\prime}$, which was previøusly a predecessor $\bullet X_{i}$, is set as the new $X_{i}$, and $X_{j}^{\prime}$ is set as the new $X_{j}$ for the next iteration (lines 8 and $9 \bullet$ Algørithm 3). Therefore, node $X_{i}$ is not visited again in the next iteratiøns. This means that there are fewer than $n$ iterations.

The •perations in lines 6-9 $\bullet$ Algørithm 3 can be completed in time $\boldsymbol{O}(\boldsymbol{n})$.

The clusters $\bullet$ several nodes must be $\bullet$ utput after each iteration. By Lemma 5 , we kn॰w that the clusters $\bullet$ all the nødes in $\mathcal{X}$ can be computed in time $\boldsymbol{0}\left(n \cdot \operatorname{width}\left(\mathcal{E}_{\mathcal{B}}\right)\right)$.

As there are fewer than $n$ iterations, Algerithm 3 can be run in time $\boldsymbol{\bullet}\left(n^{2}\right.$. $\left.\operatorname{width}\left(\mathcal{E}_{\mathcal{B}}\right)+n^{2}\right)=O\left(n^{2} \cdot \operatorname{width}\left(\mathcal{E}_{\mathcal{B}}\right)\right)$.

Lemma 9. Let $\mathcal{E}_{\mathcal{B}}$ be a valid ET •ver $\mathcal{X}=\left\{X_{1}, \ldots, X_{n}\right\}$. Swapping node $X_{i}$ and its parent $X_{p}$ in $\mathcal{E}_{\mathcal{B}}$ using Algorithm 4 and updating the clusters of $\mathcal{E}_{\mathcal{B}}$ can be completed in time $\boldsymbol{\bullet}\left(\right.$ width $\left.\left(\mathcal{E}_{\mathcal{B}}\right)\left(\left|\boldsymbol{C} \boldsymbol{h}_{\mathcal{E}_{\mathcal{B}}}\left(X_{i}\right)\right|+\left|\boldsymbol{C} \boldsymbol{h}_{\mathcal{E}_{\mathcal{B}}}\left(X_{p}\right)\right|\right)\right)$.

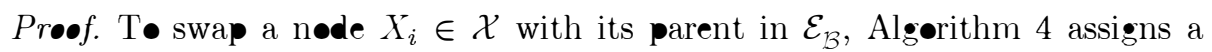

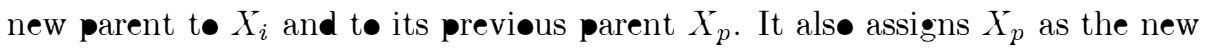
parent $\bullet$ any children $\bullet X_{i}$ wh $\bullet$ se cluster contains $X_{p}$. This can be cømpleted

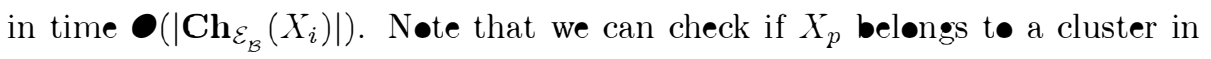
time $\boldsymbol{\bullet}(1)$.

After swapping $X_{i}$ and $X_{p}$, only the clusters $\bullet X_{i}$ and $X_{p}$ change. By Lemma 4, we kn॰w that this can be computed in $\boldsymbol{0}\left(\left|\mathbf{C h}_{\mathcal{E}_{\mathcal{B}}}\left(X_{i}\right)\right| \operatorname{width}\left(\mathcal{E}_{\mathcal{B}}\right)+\right.$ $\left.\left|\mathbf{C h}_{\mathcal{E}_{\mathcal{B}}}\left(X_{p}\right)\right| \operatorname{width}\left(\mathcal{E}_{\mathcal{B}}\right)\right)=\boldsymbol{\bullet}\left(\operatorname{width}\left(\mathcal{E}_{\mathcal{B}}\right)\left(\left|\mathbf{C h}_{\mathcal{E}_{\mathcal{B}}}\left(X_{i}\right)\right|+\left|\mathbf{C h}_{\mathcal{E}_{\mathcal{B}}}\left(X_{p}\right)\right|\right)\right)$.

Lemma 10. Let $\mathcal{E}_{\mathcal{B}}$ be a valid ET •ver $\mathcal{X}=\left\{X_{1}, \ldots, X_{n}\right\}$. Alg॰rithm 5 can be computed in time $\boldsymbol{O}\left(n^{2} \cdot\right.$ width $\left.\left(\mathcal{E}_{\mathcal{B}}\right)\right)$. 
Proof. The input of Algorithm 5 is a list of nodes $\boldsymbol{X}_{\mathrm{opt}}=\left(X_{l(1)}, \cdots, X_{l(m)}\right)$ for optimization. Assuming that these nodes are ordered from the shallowest to the deepest (i.e. the depth of $X_{l(i)}$ is greater than or equal to the depth of $\left.X_{l(i+1)}\right)$, Algorithm 5 starts swapping $X_{l(1)}$ while the width of the ET does not increase, and then it performs the same process with $X_{l(2)}, \cdots, X_{l(m)}$. Thus, the cost of Algorithm 5 is given by the cost of each swap performed during the optimization. According to Lemma 9, Algorithm 5 can be computed in time

$$
O\left(\sum_{i=1}^{m} \sum_{j=1}^{k_{i}} \operatorname{width}\left(\mathcal{E}_{\mathcal{B}}^{i, j-1}\right)\left(\left|\mathbf{C h}_{\mathcal{E}_{\mathcal{B}}^{i, j-1}}\left(X_{l(i)}\right)\right|+\left|\mathbf{C h}_{\mathcal{E}_{\mathcal{B}}^{i, j-1}}\left(\operatorname{Pa}_{\mathcal{E}_{\mathcal{B}}^{i, j-1}}\left(X_{l(i)}\right)\right)\right|\right)\right),
$$

where:

- $k_{i}<n$ is the number of times that node $X_{l(i)}$ is swapped.

- $\mathcal{E}_{\mathcal{B}}^{i, j}$ is the ET obtained after swapping node $X_{l(i)} j$ times after nodes $X_{l(1)}, \ldots, X_{l(i-1)}$ have been optimized.

- $\mathcal{E}_{\mathcal{B}}^{i, 0}=\mathcal{E}_{\mathcal{B}}^{i-1, k_{i}}$ if $\operatorname{Pa}_{\mathcal{E}_{\mathcal{B}}^{i-1, k_{i}}}\left(X_{l(i-1)}\right)=*$ (i.e., swapping node $X_{l(i-1)}$ always reduces the width of the ET candidates until its parent is the root node) and $\mathcal{E}_{\mathcal{B}}^{i, 0}=\mathcal{E}_{\mathcal{B}}^{i-1, k_{i}-1}$ otherwise.

- $\mathcal{E}_{\mathcal{B}}^{1,0}=\mathcal{E}_{\mathcal{B}}$.

When the width of a candidate $\mathcal{E}_{\mathcal{B}}^{i, j}$ is bigger than width $\left(\mathcal{E}_{\mathcal{B}}\right), \mathcal{E}_{\mathcal{B}}^{i, j}$ is rejected. Thus, by Lemma 6 , width $\left(\mathcal{E}_{\mathcal{B}}^{i, j-1}\right) \leq 2 \cdot \operatorname{width}\left(\mathcal{E}_{\mathcal{B}}\right)$, and

$$
\begin{aligned}
& \sum_{i=1}^{m} \sum_{j=1}^{k_{i}} \operatorname{width}\left(\mathcal{E}_{\mathcal{B}}^{i, j-1}\right)\left(\left|\mathbf{C h}_{\mathcal{E}_{\mathbb{B}}^{i, j-1}}\left(X_{l(i)}\right)\right|+\left|\mathbf{C h}_{\mathcal{E}_{\mathbb{B}}^{i, j-1}}\left(\operatorname{Pa}_{\mathcal{E}_{\mathcal{B}}^{i, j-1}}\left(X_{l(i)}\right)\right)\right|\right) \leq \\
& 2 \cdot \operatorname{width}\left(\mathcal{E}_{\mathcal{B}}\right)\left(\sum_{i=1}^{m} \sum_{j=1}^{k_{i}}\left|\mathbf{C h}_{\mathcal{E}_{\mathcal{B}}^{i, j-1}}\left(X_{l(i)}\right)\right|+\left|\mathbf{C h}_{\mathcal{E}_{\mathcal{B}}^{i, j-1}}\left(\operatorname{Pa}_{\mathcal{E}_{\mathcal{B}}^{i, j-1}}\left(X_{l(i)}\right)\right)\right|\right) .
\end{aligned}
$$

The complexity of Algorithm 5 can be output by counting the number of children of each $X_{l(i)}$ and its parent in each iteration. 
In any ET $\mathcal{E}_{\mathcal{B}}^{\imath, j}$, there are less than $2 n$ arcs withøut counting arcs frøm the

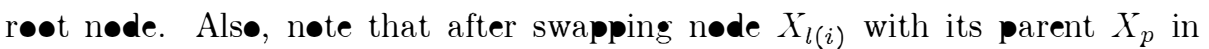
an ET $\mathcal{E}_{\mathcal{B}}^{i, j}, \mathbf{C h}_{\mathcal{E}_{\mathcal{B}}^{i, j+1}}\left(X_{p}\right) \supseteq \mathbf{C h}_{\mathcal{E}_{\mathcal{B}}^{i, j}}\left(X_{p}\right) \backslash\left\{X_{l(i)}\right\}, \mathbf{C h}_{\mathcal{E}_{\mathcal{B}}^{i, j+1}}\left(X_{l(i)}\right) \subseteq \mathbf{C h}_{\mathcal{E}_{\mathcal{B}}^{i, j}}($ $\left.X_{l(i)}\right) \backslash\left\{X_{p}\right\}$ and $\mathbf{C h}_{\mathcal{E}_{\mathcal{B}}^{i, j+1}}\left(X_{p}\right) \cup \mathbf{C h}_{\mathcal{E}_{\mathcal{B}}^{i, j+1}}\left(X_{l(i)}\right)=\mathbf{C h}_{\mathcal{E}_{\mathcal{B}}^{i, j}}\left(X_{p}\right) \cup \mathbf{C h}_{\mathcal{E}_{\mathcal{B}}^{i, j}}(l(i))$. This implies that if a nøde $X_{C}$ is the child $\bullet$ a nøde $X_{h}$ in any $\mathcal{E}_{\mathcal{B}}^{i, 0}, \ldots, \mathcal{E}_{\mathcal{B}}^{\imath, k_{i}-1}$,

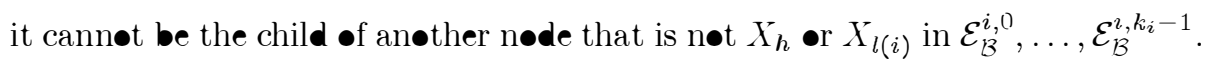
Therefore, it is evident that $\sum_{\jmath=1}^{k_{i}}\left|\mathbf{C h}_{\mathcal{E}_{\mathcal{B}}^{i, j-1}}\left(\mathbf{P a}_{\mathcal{E}_{\mathcal{B}}^{i, j-1}}\left(X_{l(i)}\right)\right)\right|<2 n$.

T• bound $\sum_{i=1}^{m} \sum_{j=1}^{k_{2}}\left|\mathbf{C h}_{\mathcal{E}_{\mathcal{B}}^{z, j-1}}\left(X_{l(i)}\right)\right|$, let us focus $\bullet$ n the number $\bullet$ children that each nøde has when it is swapped. As the nødes in $\mathcal{X}_{\bullet p t}$ are visited

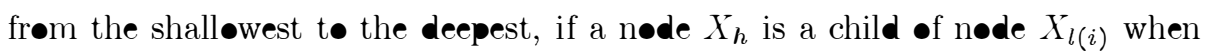

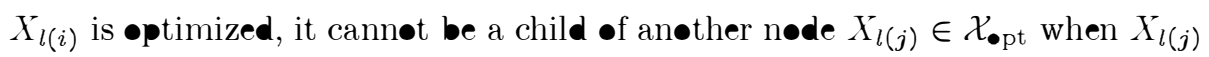
is $\bullet$ ptimized. Thus, each node $X_{h}$ can be counted less than $n$ times, and given that there are $2 n$ inner and leaf n๑des in $\mathcal{E}_{\mathcal{B}}, \sum_{i=1}^{m} \sum_{j=1}^{k_{i}}\left|\mathbf{C h}_{\mathcal{E}_{\mathcal{B}}^{2, j-1}}\left(X_{l(i)}\right)\right|<2 n^{2}$.

Finally, $2 \cdot \operatorname{width}\left(\mathcal{E}_{\mathcal{B}}\right) \cdot\left(\sum_{i=1}^{m} \sum_{j=1}^{k_{2}}\left|\mathbf{C h}_{\mathcal{E}_{\mathcal{B}}^{2, j-1}}\left(X_{l(i)}\right)\right|+\sum_{i=1}^{m} \sum_{j=1}^{k_{2}} \mid \mathbf{C h}_{\mathcal{E}_{\mathcal{B}}^{2, j-1}}(\right.$ $\left.\left.\mathbf{P a}_{\mathcal{E}_{\mathcal{B}}^{i, j-1}}\left(X_{l(i)}\right)\right) \mid\right)<\operatorname{width}\left(\mathcal{E}_{\mathcal{B}}\right)\left(4 n^{2}+4 n\right)$. Therefore, Algorithm 5 can be cœmputed in time $\boldsymbol{O}\left(n^{2} \cdot \operatorname{width}\left(\mathcal{E}_{\mathcal{B}}\right)\right)$.

Theørem 2 can be proved using the lemmas shøwn above.

Theorem 2. Let $\mathcal{E}_{\mathcal{B}}$ be a valid ET ๑ver a set $\bullet$ v variables $\mathcal{X}=\left\{X_{1}, \ldots, X_{n}\right\}$. The process described in Theorem 1 to output $\mathcal{E}_{\mathcal{B}^{\prime}}^{\prime}$ can be performed in time $\boldsymbol{O}\left(n^{2} \cdot \operatorname{width}\left(\mathcal{E}_{\mathcal{B}}\right)\right)$.

Prøof. By Lemmas 7, 8 and 10, we kn॰w that both the compilation and øptimization precess can be performed in time $\boldsymbol{O}\left(n^{2} \cdot \operatorname{width}\left(\mathcal{E}_{\mathcal{B}}\right)\right)$. 